\title{
Einstein-Kähler Metrics with Positive Ricci Curvature
}

\author{
A. Futaki, T. Mabuchi and Y. Sakane
}

\section{Introduction}

The affirmative answer, by Aubin [3] and Yau [77], to Calabi's conjecture on the existence and uniqueness of Einstein-Kähler metrics in the Ricci flat or negative case has many important consequences not only in differential geometry but also in algebraic geometry (see for instance Bourguignon et al. [17]). Their proofs fail, however, for compact complex connected manifolds $M$ with positive first Chern class $c_{1}(M)>0$, and actually a couple of obstructions are known so far concerning the existence of such metrics. The purpose of this paper is to give a brief survey of the recent progress on the existence and uniqueness problem of Einstein-Kähler metrics for $M$ as above.

In 1957, Matsushima [63] obtained the reductiveness of the group $\operatorname{Aut}(N)$ of holomorphic automorphisms of compact connected EinsteinKähler manifolds $N$ with $c_{1}(N)>0$, by showing that the Lie algebra $H^{0}(N, \mathcal{O}(T N))$ of holomorphic vector fields is a complexification of the Lie algebra of Killing vector fields on $N$. This, for instance, enabled Yau [76] to construct examples of compact Kähler manifolds with $c_{1}>0$ carrying no Einstein-Kähler metrics. In Section 1, we shall discuss these results together with Kobayashi's semistability of tangent bundle for compact Einstein-Kähler manifolds.

Inspired by the result of Kazdan and Warner $[42,43]$ on Nirenberg's problem, Futaki [25] found another obstruction $\mathcal{F}: H^{0}(M, \mathcal{O}(T M)) \rightarrow \mathbb{C}$ in 1983. This in particular allowed him to construct an example of $M$ with $\mathcal{F} \neq 0$ which admits no Einstein-Kähler metrics but has reductive $\operatorname{Aut}(M)$. A quick review of this Futaki's obstruction $\mathcal{F}$ will be given in Section 2, while we study another aspect of $\mathcal{F}$ in Section 3 from a viewpoint of symplectic geometry, following Futaki [28] and Mabuchi [58]. In

Received November 13, 1988.

Revised April 19, 1989. 
particular, given a Kähler metric in the anticanonical class, the obstruction $\mathcal{F}$ is shown to represent the barycenter of the image of the associated moment map in terms of Duistermaat-Heckman's measure.

A close relationship between the obstruction $\mathcal{F}$ and the secondary characteristic classes were pointed out by Futaki and Morita [32], which leads to the explicit formula by Futaki [27] for the group lifting of the imaginary part of $\mathcal{F}$. On the other hand, by analogy with AtiyahDonaldson's functional [22] effectively used in the study of EinsteinHermitian metrics on stable vector bundles, Mabuchi [57] defined $\mathcal{K}$ energy maps as a generalization of $\mathcal{F}$ and obtained independently a similar lifting. We shall discuss these group liftings in Section 4.

The uniqueness problem of Einstein-Kähler metrics was first treated by Berger [9] in 1965 for complex projective spaces. Later, Matsushima [64] obtained uniqueness up to biholomorphisms for all homogeneous $M$, i.e., for Kähler C-spaces. In 1985, Bando and Mabuchi [7] solved Aubin's one-parameter family of equations backwards by making essential use of the monotonicity of the $\mathcal{K}$-energy map along the solutions, and then proved, for general $M$, the uniqueness of Einstein-Kähler metrics modulo the action of the identity component of $\operatorname{Aut}(M)$. A brief survey of this uniqueness result will be given in Section 5 .

The first non-homogeneous examples of compact Einstein-Kähler manifolds with positive first Chern class were constructed by Sakane [71], while Koiso and Sakane [48] showed that $M$ admits an EinsteinKähler metric if and only if $\mathcal{F}=0$, when $M$ belongs to a certain family of compactifications of $\mathbb{C}^{*}$-bundles over compact Einstein-Kähler manifolds. We shall discuss these existence results in Section 6. Another approach (cf. Mabuchi [58]) to such results will also be given in Section 3 , and root-theoretic preliminaries for Section 6 are found in Appendix.

Finally, Section 7 is devoted to a survey of recent existence results (Siu [73], Tian [74], Tian and Yau [75]) for Einstein-Kähler metrics in the case where $M$ admits a suitable finite symmetry. Note in particular that such existence can be shown for (i) Fermat hypersurfaces of degree $m$ or $m+1$ in $\mathbb{P}^{m+1}(\mathbb{C})$, and (ii) surfaces obtained by blowing up suitable $r$ points in $\mathbb{P}^{2}(\mathbb{C})$ with $3 \leq r \leq 8$.

\section{Table of Contents}

$\S 1$. Matsushima's obstruction and Kobayashi's semistability

§2. Futaki's obstruction $\mathcal{F}$

$\S 3$. Symplectic geometry and the character $\mathcal{F}$

$\S 4$. Chern-Simons invariants and the group lifting of $\mathcal{F}$ 
$\S 5$. The uniqueness theorem

$\S 6$. Existence of Einstein-Kähler metrics I

$\S 7$. Existence of Einstein-Kähler metrics II

\& Appendix

\section{$\S 1$ Matsushima's obstruction and Kobayashi's semistability}

Let $M$ be a compact complex connected $m$-dimensional manifold endowed with a Kähler form $\omega$. We then write $\omega$ in the form

$$
\omega=\sqrt{-1} \sum_{\alpha, \beta=1}^{m} g_{\alpha \bar{\beta}} d z^{\alpha} \wedge d z^{\bar{\beta}},
$$

where $\left(z^{1}, z^{2}, \ldots, z^{m}\right)$ is a system of holomorphic local coordinates on $M$. Denote by $\Sigma R_{\alpha \bar{\beta}} d z^{\alpha} \otimes d z^{\bar{\beta}}$ the Ricci tensor of the Kähler form $\omega$. Then the associated Ricci form

$$
\operatorname{Ric}(\omega)=\sqrt{-1} \sum_{\alpha, \beta} R_{\alpha \bar{\beta}} d z^{\alpha} \wedge d z^{\bar{\beta}}
$$

satisfies $\operatorname{Ric}(\omega)=\sqrt{-1} \bar{\partial} \partial \log \operatorname{det}\left(g_{\alpha \bar{\beta}}\right)$ and represents the de Rham cohomology class $2 \pi c_{1}(M)_{\mathbb{R}}$. Define the corresponding scalar curvature $\sigma(\omega)$ and Laplacian $\square_{\omega}$ by

$$
\begin{aligned}
& \sigma(\omega):=\sum_{\alpha, \beta} g^{\bar{\beta} \alpha} R_{\alpha \bar{\beta}} \\
& \square_{\omega}:=\sum_{\alpha, \beta} g^{\bar{\beta} \alpha} \frac{\partial^{2}}{\partial z^{\alpha} \partial z^{\bar{\beta}}}
\end{aligned}
$$

where $\left(g^{\bar{\beta} \alpha}\right)$ is the inverse matrix of $\left(g_{\alpha \bar{\beta}}\right)$. Let $C^{\infty}(M)_{\mathbb{R}}, C^{\infty}(M)_{\mathbb{C}}$ be respectively the set of real, complex $C^{\infty}$ functions on $M$. Then $C^{\infty}(M)_{\mathbb{C}}$ forms a complex Lie algebra in terms of the Poisson bracket

$$
[v, w]:=\sum_{\alpha, \beta} g^{\bar{\beta} \alpha}\left(\frac{\partial v}{\partial z^{\alpha}} \frac{\partial w}{\partial z^{\bar{\beta}}}-\frac{\partial v}{\partial z^{\bar{\beta}}} \frac{\partial w}{\partial z^{\alpha}}\right) \quad v, w \in C^{\infty}(M)_{\mathbb{C}}
$$

in such a way that $\sqrt{-1} C^{\infty}(M)_{\mathbb{R}}$ forms its real Lie subalgebra. To each $v \in C^{\infty}(M)_{\mathbb{C}}$, we associate a complex $C^{\infty}$ vector field $\operatorname{grad}_{\omega} v$ on $M$, called the complex gradient of $v$, by

$$
\operatorname{grad}_{\omega} v:=\sum_{\alpha, \beta} g^{\bar{\beta} \alpha} \frac{\partial v}{\partial z^{\bar{\beta}}} \frac{\partial}{\partial z^{\alpha}}
$$


Now, let $G$ be the group $\operatorname{Aut}(M)$ of holomorphic automorphisms of $M$, and $\mathfrak{g}$ the corresponding complex Lie algebra $H^{0}(M, \mathcal{O}(T M))$. Moreover, denote by $\tilde{\mathfrak{g}}$ the space of all $v \in C^{\infty}(M)_{\mathbb{C}}$ such that $\operatorname{grad}_{\omega} v$ is holomorphic. We here recall the more or less classical fact that $\tilde{\mathfrak{g}}$ forms a complex Lie subalgebra of $C^{\infty}(M)_{\mathbb{C}}$ such that

$$
\mathcal{Y}: \tilde{\mathfrak{g}} \rightarrow \mathfrak{g}, \quad v \mapsto \mathcal{Y}(v):=-\operatorname{grad}_{\omega} v,
$$

is a complex Lie algebra homomorphism (see for instance [59]). For each $X \in \mathfrak{g}$, define the corresponding real vector field $X_{\mathbb{R}}$ by

$$
X_{\mathbb{R}}:=X+\bar{X} .
$$

Let $J$ be the complex structure of $\mathrm{M}$, and put $\mathfrak{g}_{\text {real }}:=\left\{X_{\mathbb{R}} ; X \in \mathfrak{g}\right\}$. Then by sending each $X \in \mathfrak{g}$ to $X_{\mathbb{R}} \in \mathfrak{g}_{\text {real }}$, we have the complex Lie algebra isomorphism

$$
(\mathfrak{g}, \sqrt{-1}) \cong\left(\mathfrak{g}_{\text {real }}, J\right),
$$

such that its inverse is given by

$$
X=\frac{1}{2}\left(X_{\mathbb{R}}-\sqrt{-1} J X_{\mathbb{R}}\right), \quad X \in \mathfrak{g} .
$$

For every $p$-form $\phi$ on $M$, let $X \cdot \phi, X_{\mathbb{R}} \cdot \phi$ be respectively the Lie derivative $\left(d \circ i_{X}+i_{X} \circ d\right) \phi,\left(d \circ i_{X_{\mathbb{R}}}+i_{X_{\mathbb{R}}} \circ d\right) \phi$ of $\phi$ with respect to $X, X_{\mathbb{R}}$. Then

$$
\mathcal{Y}(v)_{\mathbb{R}} \cdot \omega=-2 \sqrt{-1} \partial \bar{\partial}(\operatorname{Re} v), \quad v \in \tilde{\mathfrak{g}},
$$

where $\operatorname{Re} v \in C^{\infty}(M)_{\mathbb{R}}$ denotes the real part $\frac{1}{2}(v+\bar{v})$ of $v$. Let $\mathfrak{k}$ be the space of Killing vector fields (or equivalently infinitesimal isometries) on our Kähler manifold $M$. Then via the identification (1.1), we always regard $\mathfrak{k}$ as a real Lie subalgebra of $\mathfrak{g}$ by

$$
\mathfrak{k}=\left\{X \in \mathfrak{g} ; X_{\mathbb{R}} \cdot \omega=0\right\} .
$$

Moreover, let $K$ be the corresponding compact Lie subgroup of $G$ consisting of all holomorphic isometries on $M$.

We now consider the operator $\mathbf{L}_{\omega}: C^{\infty}(M)_{\mathbb{C}} \rightarrow C^{\infty}(M)_{\mathbb{C}}$ of Lichnerowicz [53] defined by

$$
\mathbf{L}_{\omega} v:=\left(\square_{\omega}\right)^{2} v+(\sqrt{-1} \partial \bar{\partial} v, \operatorname{Ric}(\omega))+(\bar{\partial} v, \bar{\partial} \sigma(\omega)), v \in C^{\infty}(M)_{\mathbb{C}},
$$

where $():,\{p$-forms on $M\} \times\{p$-forms on $M\} \rightarrow C^{\infty}(M)_{\mathbb{C}}, p=0,1$, $\ldots, m$, denote the natural Hermitian pairings induced from the Kähler 
form $\omega$. Then for $v_{1}, v_{2} \in C^{\infty}(M)_{\mathbb{C}}$, the $L^{2}$ inner product of $\bar{\partial}\left(\mathcal{Y}\left(v_{1}\right)\right)$, $\bar{\partial}\left(\mathcal{Y}\left(v_{2}\right)\right)$ in terms of $\omega$ is

$$
\left(\bar{\partial}\left(\mathcal{Y}\left(v_{1}\right)\right), \bar{\partial}\left(\mathcal{Y}\left(v_{2}\right)\right)\right)_{L^{2}}=\int_{M}\left(\mathbf{L}_{\omega} v_{1}\right) \bar{v}_{2} \omega^{m} / m !
$$

(see for instance Calabi $[18 ;$ p.101]), and therefore

$$
\tilde{\mathfrak{g}}=\operatorname{Ker}_{\mathbb{C}} \mathbf{L}_{\omega}:=\left\{v \in C^{\infty}(M)_{\mathbb{C}} ; \mathbf{L}_{\omega} v=0\right\}
$$

Assume now that the first Betti number $b_{1}(M)$ of $M$ is zero, where by the Kodaira vanishing theorem, this is satisfied for instance if $c_{1}(M)$ is positive. Assume further that the cohomology class $(2 \pi)^{-1}[\omega]$ represented by $(2 \pi)^{-1} \omega$ is integral. Then we have a holomorphic line bundle $L$ over $M$ and a Hermitian (fibre) metric $h_{0}$ for $L$ such that the first Chern form $c_{1}\left(L, h_{0}\right)$ of $L$ with respect to $h_{0}$ coincides with $(2 \pi)^{-1} \omega$. We shall here study the Lie algebra homomorphism $\mathcal{Y}$. First, observe that $\operatorname{Ker} \mathcal{Y}=\mathbb{C}$. Moreover,

Lemma 1.4 (see for instance [55]). $\quad \mathcal{Y}: \tilde{\mathfrak{g}} \rightarrow \mathfrak{g}$ is surjective.

Proof. For every $X=\Sigma a^{\alpha} \partial / \partial z^{\alpha} \in H^{0}(M, \mathcal{O}(T M))$, we put $\phi:=$ $-\Sigma g_{\alpha \bar{\beta}} a^{\alpha} d z^{\bar{\beta}} \in C^{\infty}(M)_{\mathbb{C}}$. Then it is easily checked that $\bar{\partial} \phi=0$. Since $b_{1}(M)=0$ implies $H^{1}(M, \mathcal{O})=\{0\}$, we now conclude that $\phi=\bar{\partial} v$, i.e., $X=-\operatorname{grad}_{\omega} v=\mathcal{Y}(v)$ for some $v \in C^{\infty}(M)_{\mathbb{C}}$, as required. Q.E.D.

It now looks quite tempting to define a good splitting of this $\mathbb{C}$-linear map $\mathcal{Y}: \tilde{\mathfrak{g}} \rightarrow \mathfrak{g}$. As to this, we actually have a very natural complex Lie algebra homomorphism

$$
u: \mathfrak{g} \rightarrow \tilde{\mathfrak{g}}, \quad X \mapsto u_{X}
$$

satisfying $\mathcal{Y}\left(u_{X}\right)=X$ for all $X \in \mathfrak{g}$ (so that $u$ is injective). To define this, we here adopt a rather conceptual and classical approach as in [59], where for our most concerned case $L=K_{M}^{-1}$, Futaki's original characterization of $u_{X}$ in terms of differential equations will be given later (see 3.1). Assume therefore the action of $G=\operatorname{Aut}(M)$ on $M$ lifts to a holomorphic bundle $G$-action on $L$. (If $L=K_{M}^{-1}$, we naturally have such a lifting.) Then the set $\mathcal{H}$ of all Hermitian metrics for $L$ admits a right $G$-action by

$$
\mathcal{H} \times G \rightarrow \mathcal{H}, \quad(h, g) \mapsto g^{*} h,
$$


where $\left(g^{*} h\right)(\ell, \ell)=h(g \cdot \ell, g \cdot \ell)$ for all $\ell \in L$. This now induces the corresponding Lie differentiation

$$
X_{\mathbb{R}} \cdot h:=\left.\frac{\partial}{\partial t}\right|_{t=0}\left(\exp \left(t X_{\mathbb{R}}\right)\right)^{*} h, \quad(X, h) \in \mathfrak{g} \times \mathcal{H} .
$$

Setting further

$$
X \cdot h:=\frac{1}{2}\left(X_{\mathbb{R}} \cdot h-\sqrt{-1} J X_{\mathbb{R}} \cdot h\right),
$$

we can now define $u_{X}$ by

$$
u_{X}=h_{0}^{-1}\left(X \cdot h_{0}\right), \quad X \in \mathfrak{g},
$$

where it is easily verified that $u_{X}$ has all the required properties (see [59]). Note that our $u_{X}$ is closely related to Futaki's obstruction via the associated moment map (see [29], [59]). Now for simplicity, denote by $u_{\mathfrak{g}}$ and $u_{\mathfrak{k}}$ the image of $\mathfrak{g}$ and $\mathfrak{k}$, respectively, under the mapping: $\mathfrak{g} \ni X \mapsto u_{X} \in \tilde{\mathfrak{g}}$. Moreover, we use the following fact in algebraic geometry: By $c_{1}(L)>0$, there exists a positive integer $r$ such that $L^{\otimes r}$ is very ample. Since the $G$-action on $L$ naturally induces a $G$-action on $V:=H^{0}\left(M, L^{\otimes r}\right)$, the group $G$ is regarded as a closed algebraic subgroup (defined over $\mathbb{C})$ of $\operatorname{Aut}\left(\mathbb{P}\left(V^{*}\right)\right)(=\operatorname{PGL}(n, \mathbb{C}))$, where $n=$ $\operatorname{dim}_{\mathbb{C}} V$ and $\mathbb{P}\left(V^{*}\right)=\left(V^{*}-\{0\}\right) / \mathbb{C}^{*}$.

We now assume that our Kähler form $\omega$ is in the de Rham cohomology class $2 \pi c_{1}(M)_{\mathbb{R}}$, and let $\operatorname{Ker}_{\mathbb{C}}\left(\square_{\omega}+1\right), \operatorname{Ker}_{i \mathbb{R}}\left(\square_{\omega}+1\right)$ be respectively the space of all $v$ in $C^{\infty}(M)_{\mathbb{C}}, \sqrt{-1} C^{\infty}(M)_{\mathbb{R}}$ such that $\left(\square_{\omega}+1\right) v=0$. Then the well-known Matsushima's theorem [63] on Einstein-Kähler metrics can be stated as follows:

Theorem 1.6. If $\operatorname{Ric}(\omega)=\omega$, then $u_{\mathfrak{g}}=\operatorname{Ker}_{\mathbb{C}}\left(\square_{\omega}+1\right)$ and $u_{\mathfrak{k}}=\operatorname{Ker}_{i \mathbb{R}}\left(\square_{\omega}+1\right)$.

Proof. By $\operatorname{Ric}(\omega)=\omega$, we have $\sigma(\omega)=m$, and the operator $\mathbf{L}_{\omega}$ of Lichnerowicz is written as

$$
\mathbf{L}_{\omega} v=\left(\square_{\omega}\right)^{2} v+\left(\square_{\omega}\right) v .
$$

Then $\tilde{\mathfrak{g}}\left(=\operatorname{Ker}_{\mathbb{C}} \mathbf{L}_{\omega}\right)$ is nothing but the space of all $v \in C^{\infty}(M)_{\mathbb{C}}$ such that $\left(\square_{\omega}+1\right) v$ is constant on $M$. Hence

$$
\operatorname{dim}_{\mathbb{C}}\left\{\operatorname{Ker}_{\mathbb{C}}\left(\square_{\omega}+1\right)\right\}=\operatorname{dim}_{\mathbb{C}}\left(\operatorname{Ker}_{\mathbb{C}} \mathbf{L}_{\omega}\right)-1=\operatorname{dim}_{\mathbb{C}} \mathfrak{g}=\operatorname{dim}_{\mathbb{C}} u_{\mathfrak{g}} .
$$


On the other hand, $\omega^{m}$ is naturally regarded as the Hermitian metric $h_{0}$ for $K_{M}^{-1}$ such that $c_{1}\left(K_{M}^{-1}, h_{0}\right)=(2 \pi)^{-1} \omega$. Therefore

$$
\begin{aligned}
\int_{M}\left(\square_{\omega}+1\right) u_{X} \omega^{m} & =\int_{M} u_{X} \omega^{m}=\int_{M}\left\{\left(X \cdot h_{0}\right) / h_{0}\right\} \omega^{m} \\
=\int_{M} X \cdot \omega^{m} & =\int_{M} d\left(i_{X} \omega^{m}\right)=0
\end{aligned}
$$

for all $X \in \mathfrak{g}$. Thus, $u_{\mathfrak{g}} \subset \operatorname{Ker}_{\mathbb{C}}\left(\square_{\omega}+1\right)$, and by comparing dimensions in this inclusion, we obtain $u_{\mathfrak{g}}=\operatorname{Ker}_{\mathbb{C}}\left(\square_{\omega}+1\right)$. Now, let $X \in \mathfrak{g}$. Then by $u_{X} \in \operatorname{Ker}_{\mathbb{C}}\left(\square_{\omega}+1\right)$,

$$
\int_{M} \operatorname{Re} u_{X} \omega^{m}=-\operatorname{Re}\left(\int_{M} \square_{\omega} u_{X} \omega^{m}\right)=0 .
$$

On the other hand, from (1.2) and (1.5), we obtain:

$$
X_{\mathbb{R}} \cdot \omega=\mathcal{Y}\left(u_{X}\right)_{\mathbb{B}} \cdot \omega=-2 \sqrt{-1} \partial \bar{\partial}\left(\operatorname{Re} u_{X}\right) .
$$

Hence by (1.3), $X \in \mathfrak{k}$ if and only if $\operatorname{Re} u_{X}=0$. This together with $u_{\mathfrak{g}}=\operatorname{Ker}_{\mathbb{C}}\left(\square_{\omega}+1\right)$ implies $u_{\mathfrak{k}}=\operatorname{Ker}_{i \mathbb{R}}\left(\square_{\omega}+1\right)$, as required. Q.E.D.

Since $\operatorname{Ker}_{\mathbb{C}}\left(\square_{\omega}+1\right)$ is a complexification of $\operatorname{Ker}_{i \mathbb{R}}\left(\square_{\omega}+1\right)$, the complex Lie algebra isomorphism

$$
\mathfrak{g} \cong u_{\mathfrak{g}}, \quad X \mapsto u_{X}
$$

allows us to regard $\mathfrak{g}$ as a complexification of $\mathfrak{k}$ in Theorem 1.6. Thus, we immediately obtain:

Corollary 1.7. If a compact complex connected manifold with positive first Chern class admits an Einstein-Kähler metric, then the full group of its holomorphic automorphisms is a reductive algebraic group (defined over $\mathbb{C}$ ).

Note that a linear algebraic group defined over $\mathbb{C}$ is called reductive if its unipotent radical is trivial. The reductiveness of such a group is characterized by the complete reducibility of its representations and is slightly stronger than the reductiveness of the corresponding Lie algebra. For instance, the additive group $\mathbb{G}_{a}$ ( $=\mathbb{C}$ as complex lie group) is not reductive as algebraic group but has a reductive Lie algebra. We next observe that several generalizations of this Matsushima's reductiveness theorem are known. For instance, Lichnerowicz [54] extended this result to compact Kähler manifolds of constant scalar curvature, 
where Calabi [18] further generalized it to extremal Kähler case. Recent Salamon's study [72] of quaternionic Kähler manifolds enables us to seek a quaternionic analogue of the reductiveness theorem (cf. [69]). Moreover, one can easily check that both 1.6 and 1.7 are valid also for compact connected Einstein-Kähler orbifolds.

Example 1.8. We here explain an example of a compact complex surface $S$, with $c_{1}(S)>0$, whose holomorphic automorphism group $\operatorname{Aut}(S)$ is not reductive (cf. Yau [76]; see also [37], [38], [70]). Let $S$ be the surface obtained by blowing up a point $p$ of the complex projective plane $\mathbb{P}^{2}(\mathbb{C})$. Then, clearly $c_{1}(S)>0$, and since we may assume $p=(1$ : $0: 0)$ without loss of generality, the group $\operatorname{Aut}(S)$ is identified with the isotropy subgroup of $\mathrm{PGL}(3, \mathbb{C})$ at $(1: 0: 0)$ :

$$
\operatorname{Aut}(S) \cong\left\{g=\left(g_{i j}\right)_{1 \leqslant i, j \leqslant 3} \in \operatorname{PGL}(3, \mathbb{C}) ; g_{21}=g_{31}=0\right\}
$$

Hence $\operatorname{Aut}(S)$ is not reductive, because the right-hand side in the above has the non-trivial unipotent radical

$$
\left\{g=\left(g_{i j}\right) \in \operatorname{PGL}(3, \mathbb{C}) ; g_{i j}=\delta_{i j} \text { for } i \neq 1\right\} \cong \mathbb{G}_{a}^{2}\left(=\mathbb{C}^{2}\right)
$$

Recall that a Hermitian symmetric space of compact type decomposes into a product of irreducible ones. We shall now show that a similar decomposition is true also for Einstein-Kähler manifolds with positive first Chern class. Let $E$ be a holomorphic vector bundle of rank $\operatorname{rk}(E)$ over an $m$-dimensional compact connected Kähler manifold $(M, \omega)$, where $\omega$ is the Kähler form on $M$. Then $E$ is said to be $\omega$-stable (in the sense of Mumford-Takemoto) if the following inequality holds for all coherent subsheaves $\mathcal{S}$ of $\mathcal{O}(E)$ with $0<\operatorname{rk}(\mathcal{S})<\operatorname{rk}(E)$ :

$$
\frac{\int_{M} c_{1}(\mathcal{S}) \wedge \omega^{m-1}}{\operatorname{rk}(\mathcal{S})}<\frac{\int_{M} c_{1}(E) \wedge \omega^{m-1}}{\operatorname{rk}(E)}
$$

Now, the semistability theorem of Kobayashi [46] and Lübke [56] asserts that, if $\mathrm{E}$ admits an Einstein-Hermitian metric, then $\mathrm{E}$ is a direct sum of holonomy-irreducible stable vector bundles. On the other hand, a theorem of Kobayashi [44] (together with Yau's affirmative solution [77] of Calabi's conjecture) shows that $M$ is simply connected if $c_{1}(M)>$ 0 . Hence, the de Rham decomposition for Kähler manifolds (see for instance [47]) is interpreted as follows: 
Theorem 1.9. Let $(M, \omega)$ be an Einstein-Kähler manifold with $c_{1}(M)>0$. Then there exist simply connected Einstein-Kähler manifolds $\left(M_{i}, \omega_{i}\right), 1 \leqq i \leqq r$, with $c_{1}\left(M_{i}\right)>0$ such that

(1) $M=M_{1} \times \ldots \times M_{r}$;

(2) each $T M_{i}$ is $\omega_{i}$-stable;

(3) $\omega=\Sigma_{i=1}^{r} \operatorname{pr}_{i}^{*}\left(\omega_{i}\right)$,

where $\operatorname{pr}_{i}: M\left(=M_{1} \times \ldots \times M_{r}\right) \rightarrow M_{i}$ denotes the natural projection to the $i$-th factor.

\section{$\S 2 . \quad$ Futaki's obstruction $\mathcal{F}$}

In this section, we retain the notation of $\S 1$. The covariant and contravariant derivatives are respectively denoted by lower and upper indices after a comma, and we assume the summation convention if there is no fear of confusion. For instance, if $X=\sum X^{\alpha} \partial / \partial z^{\alpha}$ is a holomorphic vector field, then

$$
X^{\alpha},_{\bar{\beta}}=0 .
$$

The Ricci identity is expressible as

$$
\xi_{\alpha, \beta \bar{\gamma}}=\xi_{\alpha, \bar{\gamma} \beta}+R_{\alpha}^{\delta} \bar{\gamma} \beta \xi_{\delta}
$$

for a 1-form $\sum \xi_{\alpha} d z^{\alpha}$. Recall also that, for any smooth function $u$, we have the commutation relations: $u,_{\alpha \beta}=u,_{\beta \alpha}$ and $u,{ }_{\alpha \bar{\beta}}=u, \bar{\beta} \alpha$. Now, we begin with the following well-known fact (see [35] for a proof).

Lemma 2.1. On a compact Kähler manifold, if $\sigma$ is a real exact smooth $(p, p)$-form, there is a real smooth $(p-1, p-1)$-form $\tau$, unique up to a $\partial \bar{\partial}$-closed form, such that

$$
\sigma=\sqrt{-1} \partial \bar{\partial} \tau
$$

Let $M$ be an $m$-dimensional compact complex connected manifold, and let $\mathcal{K}$ be the set of all $C^{\infty}$ Kähler forms which represent $2 \pi c_{1}(M)_{\mathbb{R}}$. Take an $\omega \in \mathcal{K}$. Since the Ricci form $\operatorname{Ric}(\omega)$ represents $2 \pi c_{1}(M)_{\mathbb{R}}$, the $(1,1)$-form $\operatorname{Ric}(\omega)-\omega$ is exact. By Lemma 2.1, there is a real-valued smooth function $f_{\omega}$, unique up to an additive constant, such that

$$
\operatorname{Ric}(\omega)-\omega=\sqrt{-1} \partial \bar{\partial} f_{\omega} .
$$


Therefore $\omega$ is an Einstein-Kähler form if and only if $f_{\omega}$ is constant. We now define a linear functional $\mathcal{F}: H^{0}(M, \mathcal{O}(T M))(=\mathfrak{g}) \rightarrow \mathbb{C}$ by

$$
\mathcal{F}(X)=\frac{(m+1) \sqrt{-1}}{(2 \pi)^{m+1}} \int_{M}\left(X f_{\omega}\right) \omega^{m} .
$$

Theorem 2.2 (Futaki [25]). The functional $\mathcal{F}$ does not depend on the choice of $\omega \in \mathcal{K}$. In particular $\mathcal{F}$ depends only on the complex structure of $M$, and is invariant under the coadjoint action of $G(=$ $\operatorname{Aut}(M))$; this implies that $\mathcal{F}$ is a complex Lie algebra homomorphism of $\mathfrak{g}$ into $\mathbb{C}$. If $M$ admits an Einstein-Kähler metric, then $\mathcal{F}=0$.

Proof. If we take $\omega_{0}$ and $\omega_{1}$ in $\mathcal{K}$, there is $\phi \in C^{\infty}(M)_{\mathbb{R}}$ such that $\omega_{1}=\omega_{0}+\sqrt{-1} \partial \bar{\partial} \phi$. Put $\omega_{t}=\omega_{0}+t \sqrt{-1} \partial \bar{\partial} \phi, 0 \leq t \leq 1$, and let $\mathcal{F}_{t}$ be the corresponding family of functionals obtained from $\mathcal{F}$ by substituting $\omega_{t}$ for $\omega$. It is then sufficient to show

$$
\frac{d}{d t} \mathcal{F}_{t}(X)=0, \quad 0 \leq t \leq 1 .
$$

For the time being, denote $f_{\omega_{t}}$ by $f_{t}$ for simplicity. Since

$$
\sqrt{-1} \partial \bar{\partial} f_{t}=\operatorname{Ric}\left(\omega_{t}\right)-\omega_{t}=-\sqrt{-1} \partial \bar{\partial}\left(\log \frac{\omega_{t}^{m}}{\omega_{0}^{m}}-f_{0}+t \phi\right),
$$

we may assume

$$
f_{t}=f_{0}-t \phi-\log \frac{\omega_{t}^{m}}{\omega_{0}^{m}} .
$$

From now on, $\omega_{t}$ and $f_{t}$ are written as $\omega$ and $f$ for notational convenience, and the covariant derivatives are taken with respect to the Kähler form $\omega_{t}$. Moreover by the symbol $\sim$, we denote the congruence modulo divergence terms. Then for $X \in \mathfrak{g}$,

$$
\frac{d}{d t}\left(X f \omega^{m}\right)=X(-\phi-\square \phi) \omega^{m}+(X f) \square \phi \omega^{m} .
$$

The Ricci identity together with $X^{\gamma}, \bar{\beta}=0$ yields

$$
\begin{aligned}
-X(\square \phi+\phi) & =-X^{\gamma}\left(g^{\bar{\beta} \alpha} \phi,_{\alpha \bar{\beta} \gamma}+\phi,_{\gamma}\right) \\
& =-X^{\gamma}\left(g ^ { \overline { \beta } \alpha } \left(\phi,_{\alpha \gamma \bar{\beta}}+R_{\alpha}{ }^{\delta}{ }_{\gamma \bar{\beta}} \phi, \delta\right.\right. \\
& \left.\sim \phi_{,_{\gamma}}\right) \\
& \sim X^{\gamma} R_{\gamma}{ }^{\delta} \phi,_{\delta}-X^{\gamma} \phi_{,_{\gamma}},
\end{aligned}
$$


while the identities $X^{\gamma},_{\bar{\beta}}=0$ and $R_{\alpha \bar{\beta}}-g_{\alpha \bar{\beta}}=f,_{\alpha \bar{\beta}}$ imply

$$
\begin{aligned}
(X f) \square \phi & =X^{\gamma} f_{,_{\gamma}} g^{\bar{\beta} \alpha} \phi,_{\alpha \bar{\beta}} \sim-X^{\gamma} f,_{\gamma \bar{\beta}} g^{\bar{\beta} \alpha} \phi,_{\alpha} \\
& =-X^{\gamma} R_{\gamma}{ }^{\alpha} \phi,_{\alpha}+X^{\gamma} \phi,_{\alpha} .
\end{aligned}
$$

Thus $-X(\square \phi+\phi)+X f \square \phi$ is of divergence form, and its integration with the volume form $\omega^{m}$ vanishes by the divergence theorem. Thus the functional $\mathcal{F}$ does not depend on the choice of $\omega \in \mathcal{K}$. This implies that $\mathcal{F}$ depends only on the complex structure of $M$. Furthermore, for $a \in G$,

$$
\begin{aligned}
\mathcal{F}\left(a_{*} X\right) & =\frac{(m+1) \sqrt{-1}}{(2 \pi)^{m+1}} \int_{M}\left(a_{*} X\right) f \omega^{m} \\
& =\frac{(m+1) \sqrt{-1}}{(2 \pi)^{m+1}} \int_{M} X\left(a^{*} f\right) a^{*} \omega^{m}=\mathcal{F}(X)
\end{aligned}
$$

in view of

$$
\operatorname{Ric}\left(a^{*} \omega\right)-a^{*} \omega=a^{*} \operatorname{Ric}(\omega)-a^{*}(\omega)=\sqrt{-1} \partial \bar{\partial} a^{*} f
$$

In particular $\mathcal{F}([X, Y])=0$ for all $X, Y \in \mathfrak{g}$, from which it follows that $\mathcal{F}$ is a Lie algebra homomorphism into $\mathbb{C}$. If $M$ admits an EinsteinKähler form $\omega \in \mathcal{K}$, then we have $\mathcal{F}=0$ by $f_{\omega}=0$. Q.D.

Theorem 2.2 was obtained as a generalization of the integrability condition of Kazdan and Warner for Nirenberg's problem (see [42], [43], [41]). Bourguignon [16] also generalized the condition of Kazdan and Warner to obtain various conservation laws in conformal geometry.

We can see that $\mathcal{F}$ belongs to the intersection of two families of invariants. The one is the family of Kählerian invariants ([5], [18], [26]), and the other is the family of invariants for compact complex manifolds ([31]). To explain the first, let $M$ be a compact Kähler manifold with Kähler class $\Omega$, where we mean by Kähler class the de Rham cohomology class of the Kähler form. Let $\mathcal{K}_{\Omega}$ be the space of all Kähler forms in $\Omega$. Choosing any Kähler form $\omega$ in $\mathcal{K}_{\Omega}$, let $c_{k}(\omega)$ and $H c_{k}(\omega)$ be respectively the $k$-th Chern form and its harmonic part. By Lemma 2.1, there is a real smooth $(k-1, k-1)$-form $f_{k}$ such that

$$
c_{k}(\omega)-H c_{k}(\omega)=\sqrt{-1} \partial \bar{\partial} f_{k}
$$

We define a linear functional $\mathcal{F}_{k}: \mathfrak{g} \rightarrow \mathbb{C}$ by

$$
\mathcal{F}_{k}(X)=\frac{(m+1) ! \sqrt{-1}}{k !(m-k+1) !(2 \pi)^{m+1}} \int_{M} L_{X} f_{k} \wedge \omega^{m-k+1}
$$


where $L_{X}$ denotes the complex Lie differentiation $i_{X} \circ d+d \circ i_{X}$ with respect to the holomorphic vector field $X \in \mathfrak{g}$. We emphasize that, in this definition, it is not necessary to assume $c_{1}(M)>0$.

Theorem 2.3. $\quad \mathcal{F}_{k}$ is independent of the choice of $\omega$ in $\mathcal{K}_{\Omega}$. In particular, $\mathcal{F}_{k}$ is invariant under the coadjoint action of the subgroup $G_{\Omega}$ of $G$ consisting of all automorphisms which preserve $\Omega$. If $M$ admits $\omega \in \mathcal{K}_{\Omega}$ such that $c_{k}(\omega)=H c_{k}(\omega)$, then $\mathcal{F}_{k}=0$.

Theorem 2.3 is due to Bando [5] and is proven in the same way as in Theorem 2.2. Note that $\mathcal{F}_{k}$ is an invariant of $(M, \Omega)$; more precisely $\mathcal{F}_{k}$ varies as $\Omega$ does. If $c_{1}(M)>0$, we may take $\Omega$ to be $2 \pi c_{1}(M)_{\mathbb{R}}$, and the case where $k=1$ recovers Theorem 2.2. The following interesting example is due to Calabi [18].

Example 2.4. Let $M$ be the rational surface obtained from the complex projective plane by blowing up three noncollinear points, say $(1: 0: 0),(0: 1: 0)$ and $(0: 0: 1)$. The identity component $G^{0}$ of $G=\operatorname{Aut}(M)$ is isomorphic to the subgroup of PGL $(3, \mathbb{C})$ consisting of all elements represented by the diagonal matrices, and thus $\mathfrak{g}$ is isomorphic to the corresponding Lie subalgebra $\cong \mathfrak{s l}(3, \mathbb{C})$. $G$ also contains the symmetric group $\mathfrak{S}_{3}$ of degree 3 , as a subgroup, which acts on $M$ by the permutations of the three exceptional curves and descends to the permutations of the three coordinates of the projective plane. If we take $\Omega=2 \pi c_{1}(M)_{\mathbb{R}}$, then $\Omega$ is preserved by $G$, and in particular by $\mathfrak{S}_{3}$, so that we see $\mathcal{F}=0$ by the invariance of $\mathcal{F}$ under the coadjoint action of $G$. On the other hand if $\Omega \neq 2 \pi c_{1}(M)_{\mathbb{R}}, G_{\Omega}$ can be smaller than $G$. Actually, Calabi showed that, for some positive class $\Omega$, one has $\mathcal{F}_{1} \neq 0$.

To explain the second family let $M$ be again a compact complex manifold with $c_{1}(M)>0$. The Calabi-Yau theorem [77] says that, given a real $(1,1)$-form $\omega \in \mathcal{K}$ representing $2 \pi c_{1}(M)_{\mathbb{R}}$, we have a unique Kähler form $\eta \in \mathcal{K}$ such that

$$
\omega=\operatorname{Ric}(\eta) .
$$

We shall rewrite $\mathcal{F}$ in terms of $\eta$. Then we have

$$
\sqrt{-1} \partial \bar{\partial} f_{\omega}=\operatorname{Ric}(\omega)-\operatorname{Ric}(\eta)=-\sqrt{-1} \partial \bar{\partial} \log \frac{\omega^{m}}{\eta^{m}},
$$

so that we may assume

$$
f_{\omega}=-\log \frac{\omega^{m}}{\eta^{m}}
$$


We therefore obtain

$$
\begin{aligned}
\mathcal{F}(X) & =-\frac{(m+1) \sqrt{-1}}{(2 \pi)^{m+1}} \int_{M} X\left(\log \frac{\omega^{m}}{\eta^{m}}\right) \omega^{m} \\
& =-\frac{(m+1) \sqrt{-1}}{(2 \pi)^{m+1}} \int_{M} X\left(\frac{\omega^{m}}{\eta^{m}}\right) \eta^{m} \\
& =\frac{(m+1) \sqrt{-1}}{(2 \pi)^{m+1}} \int_{M} \operatorname{div}_{\eta}(X) \operatorname{Ric}(\eta)^{m}
\end{aligned}
$$

where $\operatorname{div}_{\eta}(X)$ denotes the complex divergence $\left(X \cdot \eta^{m}\right) / \eta^{m}$ of $X$ with respect to the Kähler form $\eta$, and the last identity is obtained by integration by parts (cf. [32]).

Remark 2.5. In $\S 1$, the complex divergence $\operatorname{div}_{\eta}(X)$ with respect to $\eta$ was denoted by $u_{X}$. The above computation shows that

$$
\mathcal{F}(X)=\frac{(m+1) \sqrt{-1}}{(2 \pi)^{m+1}} \int_{M} u_{X} \omega^{m}
$$

We notice at this stage that, for definition of $\mathcal{F}$, we need to assume neither that $c_{1}(M)$ is positive nor that $M$ is Kählerian. Let $M$ be a compact complex manifold and take a Hermitian metric $g$ for $M$. Let $\nabla$ be the Hermitian connection for $T M$ induced by the Hermitian metric $g$ and let $L_{X}$ be the Lie differentiation for vector fields with respect to $X \in \mathfrak{g}$, i.e., $L_{X}(Y)=[X, Y]$. We then define $\mathcal{F}$ by

$$
\mathcal{F}(X)=\frac{(m+1) \sqrt{-1}}{(2 \pi)^{m+1}} \int_{M} \operatorname{div}(X) \operatorname{Ric}(g)^{m}
$$

where

$$
\begin{aligned}
\operatorname{div}(X) & =\operatorname{Tr} \mathcal{L}(X), \mathcal{L}(X)=\nabla_{X}-L_{X} \\
\operatorname{Ric}(g) & =-\sqrt{-1} \partial \bar{\partial} \log \operatorname{det}(g)
\end{aligned}
$$

One can now verify that $\mathcal{F}$ defined by $(2.6)$ is independent of the choice of the Hermitian metric $g$. Viewing $T M$ as a holomorphic vector bundle, we denote by $\Theta$ the curvature form for $\nabla$ on $T M$. Then (2.6) is rewritten as

$$
\mathcal{F}(X)=\left(\frac{\sqrt{-1}}{2 \pi}\right)^{m+1} \int_{M}(m+1)(\operatorname{Tr} \mathcal{L}(X))(\operatorname{Tr} \Theta)^{m}
$$


More generally, let $\phi$ be a $\mathrm{GL}(m, \mathbb{C})$-invariant symmetric polynomial of degree $m+k$, and define

$$
\mathcal{F}_{\phi}(X)=\int_{M} \phi(\mathcal{L}(X)+\Theta)
$$

It can be proven in the same way as in Theorem 2.2 that $\mathcal{F}_{\phi}$ is independent of the choice of the Hermitian metric $g$. We can therefore regard $\mathcal{F}_{\phi}$ as a $G$-invariant polynomial. The case where $\phi=c_{1}^{m+1}$ reduces to the original $\mathcal{F}$.

We now notice that $\mathcal{F}_{\phi}$ was already in the proof of the localization formula for Chern numbers due to Bott [12]. By the same arguments as in [12], one can also give a localization formula for $\mathcal{F}_{\phi}(X)$ in terms of the zero set of $X$. In view of these facts, the close relation of $\mathcal{F}$ with the Chern-Simons invariants and the equivariant cohomology was observed in [31]. Almost at the same period, the invariant polynomial $\mathcal{F}_{\phi}$ was extensively studied, mainly for real manifolds, in the context of symplectic geometry (see Atiyah and Bott [2], Berline and Vergne [10], Duistermaat and Heckman [23]), which led us to make further studies of $\mathcal{F}$ from wider viewpoints (cf. [32], [58], [59] and §3).

For localization, assume that the zero set $\operatorname{Zero}(X)$ of $X$ consists of complex submanifolds $Z_{\lambda}, \lambda \in \Lambda$, of $M$. Let $\nu\left(Z_{\lambda}\right)$ be the normal bundle of $Z_{\lambda}$ in $M$. Then the restriction of $\mathcal{L}(X)$ to $Z_{\lambda}$ induces a holomorphic section $L_{\lambda}(X)$ of the endomorphism bundle of $\nu\left(Z_{\lambda}\right)$, which represents nothing but the infinitesimal isotropy action of $X$ on $\nu\left(Z_{\lambda}\right)$. The formula of Bott in our case can be stated as follows (see [31], [29]):

Theorem 2.7. If $\mathcal{L}_{\lambda}(X) \in \operatorname{GL}\left(\nu\left(Z_{\lambda}\right)_{q}\right)$ for all $q \in \operatorname{Zero}(X)$, then we have

$$
\mathcal{F}_{\phi}(X)=\sum_{\lambda} \int_{Z_{\lambda}} \phi\left(\mathcal{L}(X)+\Theta_{\mid Z_{\lambda}}\right) / \operatorname{det}\left\{\frac{\sqrt{-1}}{2 \pi}\left(\mathcal{L}_{\lambda}(X)+\Psi_{\lambda}\right)\right\}
$$

where $\Psi_{\lambda}$ denotes the curvature form of $\nu\left(Z_{\lambda}\right)$ with respect to the Hermitian metric induced by the metric $g$.

We shall now give examples of how to compute $\mathcal{F}$. Let $n_{1}$ and $n_{2}$ be positive integers, and $L_{i}$ be the tautological line bundle $\mathcal{O}(-1)$ over the complex projective space $\mathbb{P}^{n_{i}}(\mathbb{C})$ of dimension $n_{i}$ for $i=1,2$. Denote by $p_{i}: \mathbb{P}^{n_{1}}(\mathbb{C}) \times \mathbb{P}^{n_{2}}(\mathbb{C}) \rightarrow \mathbb{P}^{n_{i}}(\mathbb{C})$ the projection to the $i$-th factor. Let $M$ be the total space $\mathbb{P}(E)$ of the projective bundle $p r: \mathbb{P}(E) \rightarrow N$ associated with the 2-plane bundle

$$
E=p_{1}^{*} L_{1} \oplus p_{2}^{*} L_{2} \rightarrow N:=\mathbb{P}^{n_{1}}(\mathbb{C}) \times \mathbb{P}^{n_{2}}(\mathbb{C})
$$


Then the proper surjective holomorphic map pr: $M \rightarrow N$ induces the Lie algebra homomorphism $\mathrm{pr}_{*}: \mathfrak{g} \rightarrow \mathfrak{g}^{\prime}$, where $\mathfrak{g}:=H^{0}(M, \mathcal{O}(T M))$ and $\mathfrak{g}^{\prime}:=H^{0}(N, \mathcal{O}(T N))$. We can then express $G^{0}:=\operatorname{Aut}^{0}(M)$ in the following form (see [71] for a proof):

Proposition 2.8. Let $M, \mathfrak{g}, \mathfrak{g}^{\prime}$ be as above. Then $c_{1}(M)>0$. Furthermore, $G^{0} \cong \operatorname{PGL}\left(n_{1}+1, \mathbb{C}\right) \times \operatorname{PGL}\left(n_{2}+1, \mathbb{C}\right) \times \mathbb{C}^{*}$. In particular, $\mathfrak{g}$ splits into a direct sum of Lie subalgebras

$$
\mathfrak{g}=\mathfrak{s l}\left(n_{1}+1, \mathbb{C}\right) \oplus \mathfrak{s l}\left(n_{2}+1, \mathbb{C}\right) \oplus \mathbb{C}
$$

where the direct summand $\mathbb{C}$ stands for the kernel of $\mathrm{pr}_{*}: \mathfrak{g} \rightarrow \mathfrak{g}^{\prime}$.

By 2.8 above, $M$ satisfies Matsushima's necessary condition for the existence of Einstein-Kähler metrics (cf. §1). To write explicitly a generator for $\mathbb{C}$ in (2.8.1), we choose local coordinates on $M$ as follows. Let $z=\left(z_{0}: \cdots: z_{n_{1}}\right)$ and $w=\left(w_{0}: \cdots: w_{n_{2}}\right)$ be respectively the systems of homogeneous coordinates for $\mathbb{P}^{n_{1}}(\mathbb{C})$ and $\mathbb{P}^{n_{2}}(\mathbb{C})$. Consider the open sets $U_{1}=\left\{z_{0} \neq 0\right\}$ in $\mathbb{P}^{n_{1}}(\mathbb{C})$ and $U_{2}=\left\{w_{0} \neq 0\right\}$ in $\mathbb{P}^{n_{2}}(\mathbb{C})$. Moreover, consider meromorphic sections $\sigma_{1}, \sigma_{2}$ for $L_{1}, L_{2}$ having neither zeroes nor' poles on $U_{1}, U_{2}$ respectively. Note that, when restricted to $U_{1} \times U_{2}$, our $M$ is isomorphic to $U_{1} \times U_{2} \times \mathbb{P}^{1}(\mathbb{C})$. We then have homogeneous coordinates $\left(\xi_{1}: \xi_{2}\right)$ on the third factor $\mathbb{P}^{1}(\mathbb{C})$ such that $\xi_{1} p_{1}^{*} \sigma_{1}(z, w)+\xi_{2} p_{2}^{*} \sigma_{2}(z, w)$ on $E$ projects down to $\left(z, w,\left(\xi_{1}: \xi_{2}\right)\right) \in M$ for all $(z, w) \in U_{1} \times U_{2}$. Now, on the open subset $V=\left\{\xi_{1} \neq 0\right\}$ of $\mathbb{P}^{1}(\mathbb{C})$, choose the inhomogeneous coordinate $\zeta=\xi_{2} / \xi_{1}$. Then on $U_{1} \times U_{2} \times V$, a $\mathbb{C}$-base $X$ for the Lie algebra $\mathbb{C}$ in $(2.8 .1)$ is written in the form

$$
X=2 \pi \sqrt{-1} \zeta \frac{\partial}{\partial \zeta} .
$$

Proposition 2.9. If $n_{1}=n_{2}$, then $\mathcal{F}=0$.

Proof. Since $\mathcal{F}$ vanishes on $[\mathfrak{g}, \mathfrak{g}]=\mathfrak{s l}\left(n_{1}+1, \mathbb{C}\right) \oplus \mathfrak{s l}\left(n_{2}+1, \mathbb{C}\right)$, it follows that $\mathcal{F}=0$ if and only if $\mathcal{F}(X)=0$ for $X$ as above. Let $n:=n_{1}=n_{2}$. We can then define an automorphism $a \in G$ by

$$
a\left(z, w,\left(\xi_{1}: \xi_{2}\right)\right)=\left(w, z,\left(\xi_{2}: \xi_{1}\right)\right)
$$

in terms of the coordinates above. In view of the $G$-invariance of $\mathcal{F}$, we have $\mathcal{F}(X)=\mathcal{F}\left(a_{*}(X)\right)=-\mathcal{F}(X)$, and therefore $\mathcal{F}(X)=0$, as required.

Q.E.D.

Proposition 2.10. If $n_{1}=1$ and $n_{2}=2$, then $\mathcal{F} \neq 0$. 
Proof. By setting $\eta=\zeta^{-1}$, we have

$$
\frac{\sqrt{-1}}{2 \pi} X=-\zeta \frac{\partial}{\partial \zeta}=\eta \frac{\partial}{\partial \eta}
$$

so that the zero set of $X$ consist of two connected components

$$
\begin{aligned}
& Z_{0}=\{\eta=0\}=\left\{\xi_{1}=0\right\} \cong \mathbb{P}^{1} \times \mathbb{P}^{2} \\
& Z_{\infty}=\{\zeta=0\}=\left\{\xi_{2}=0\right\} \cong \mathbb{P}^{1} \times \mathbb{P}^{2}
\end{aligned}
$$

Hence by (1),

$$
\frac{\sqrt{-1}}{2 \pi} \mathcal{L}_{\lambda}(X)= \pm 1
$$

as an endomorphism of $\nu\left(Z_{\lambda}\right)$, where the plus or minus sign is respectively taken according as $\lambda=0$ or $\infty$. Furthermore, the normal bundles $\nu\left(Z_{0}\right)$ and $\nu\left(Z_{\infty}\right)$ are given by

$$
\nu\left(Z_{0}\right)=L_{0}=\nu\left(Z_{\infty}\right)^{-1},
$$

where $L_{0}:=p_{1}^{*} L_{1} \otimes p_{2}^{*} L_{2}^{-1}$. Let $a$ and $b$ be respectively the positive generators for $H^{2}\left(\mathbb{P}^{1} ; \mathbb{Z}\right)$ and $H^{2}\left(\mathbb{P}^{2} ; \mathbb{Z}\right)$. Then

$$
a^{2}=b^{3}=0, a\left[\mathbb{P}^{1}\right]=b^{2}\left[\mathbb{P}^{2}\right]=1 \text {. }
$$

Clearly, by (2), the cohomology class represented by $\nu\left(Z_{\lambda}\right)$ is $p_{1}^{*} a-p_{2}^{*} b$ if $\lambda=0$, and is $p_{2}^{*} b-p_{1}^{*} a$ if $\lambda=-\infty$ (cf. Theorem 2.7). Moreover,

$$
\frac{\sqrt{-1}}{2 \pi} \operatorname{Tr}(\mathcal{L}(X)+\Theta)_{\mid Z_{\lambda}}
$$

represents the cohomology class

$$
\frac{\sqrt{-1}}{2 \pi} \mathcal{L}_{\lambda}(X)+c_{1}(M)_{\mid Z_{\lambda}}=\frac{\sqrt{-1}}{2 \pi} \mathcal{L}_{\lambda}(X)+c_{1}\left(\nu\left(Z_{\lambda}\right)\right)+c_{1}\left(Z_{\lambda}\right)
$$

which is $1+3 p_{1}^{*} a+2 p_{2}^{*} b$ or $-1+p_{1}^{*} a+4 p_{2}^{*} b$, according as $\lambda=0$ or $\infty$. Now by applying Theorem 2.7 , we conclude that

$$
\begin{aligned}
\mathcal{F}(X) & =\left(\frac{\left(1+3 p_{1}^{*} a+2 p_{2}^{*} b\right)^{4}}{1+p_{1}^{*} a-p_{2}^{*} b}+\frac{\left(-1+p_{1}^{*} a+4 p_{2}^{*} b\right)^{4}}{-1-p_{1}^{*} a+p_{2}^{*} b}\right)\left[\mathbb{P}^{1} \times \mathbb{P}^{2}\right] \\
& =400 \neq 0 .
\end{aligned}
$$




\section{$\S 3$. Symplectic geometry and the character $\mathcal{F}$}

Let $M$ be a compact complex connected manifold with $c_{1}(M)>0$. Throughout this section, we fix a Kähler form $\omega$ representing $2 \pi c_{1}(M)_{\mathbb{R}}$. Let us keep the same notation as in the previous sections except that we write $\square_{\omega}$ (cf. $\left.\S 1\right)$ and $f_{\omega}$ (cf. $\S 2$ ) simply as $f$ and $\square$ respectively. Let $P_{f}: C^{\infty}(M)_{\mathbb{C}} \rightarrow C^{\infty}(M)_{\mathbb{C}}$ be the second order differential operator defined by

$$
P_{f} v:=\square v+\sum_{\alpha, \beta} g^{\bar{\beta} \alpha} \frac{\partial f}{\partial z^{\alpha}} \frac{\partial v}{\partial z^{\bar{\beta}}}=\square v-\mathcal{Y}(v) f .
$$

Consider the Hermitian inner product $\ll, \gg$ with respect to the weighted volume form $e^{f} \omega^{m}$ :

$$
\ll u, v \gg=\int_{M} u \bar{v} e^{f} \omega^{m}, \quad u, v \in C^{\infty}(M)_{\mathbb{C}},
$$

Then the operator $P_{f}$ is selfadjoint with respect to $\ll, \gg$, and the eigenvalues of $P_{f}$ are all nonpositive real numbers, since

$$
\ll P_{f} u, v \gg=-\int_{M}(\bar{\partial} u, \bar{\partial} v)_{\omega} e^{f} \omega^{m}=\ll u, P_{f} v \gg .
$$

We now put $\{u, v\}:=\left(\bar{\partial}\left\{\left(P_{f}+1\right) u\right\}, \bar{\partial} v\right)_{\omega}+(\bar{\partial} \mathcal{Y}(u), \bar{\partial} \mathcal{Y}(v))_{\omega}$. Then integration by parts together with the Ricci identity yields

$$
\int_{M}\{u, v\} e^{f} \omega^{m}=0, \quad u, v \in C^{\infty}(M)_{\mathbb{C}} .
$$

Recall, by definition, we have $\mathfrak{g}=H^{0}(M, \mathcal{O}(T M))$. As a corollary of the formula (3.1.2), it follows that:

Proposition 3.1 ([28], [31]). Let $-\lambda_{1}$ be the first nonzero eigenvalue of $P_{f}$. Then $\lambda_{1} \geq 1$. Furthermore, by sending $v \in \operatorname{Ker} \mathbb{C}\left(P_{f}+1\right)$ to $\mathcal{Y}(v)$ (cf. $\S 1)$, we have an isomorphism $\operatorname{Ker}_{\mathbb{C}}\left(P_{f}+1\right) \cong \mathfrak{g}$.

Proof. Let $0 \neq v \in C^{\infty}(M)_{\mathbb{C}}$ be such that $P_{f} v+\lambda_{1} v=0$. Then by setting $u=v$ in (3.1.2), we obtain

$$
\int_{M}\left(\left(1-\lambda_{1}\right)|\bar{\partial} v|_{\omega}^{2}+|\bar{\partial} \mathcal{Y}(v)|_{\omega}^{2}\right) e^{f} \omega^{m}=0,
$$

which implies $\lambda_{1} \geq 1$ and moreover, for $\lambda_{1}=1$, the vector field $\mathcal{Y}(v)$ is holomorphic. Since $\left(P_{f}+1\right)(v+C)-\left(P_{f}+1\right) v=C \neq 0$ for $0 \neq C \in \mathbb{C}$, 
the mapping $\operatorname{Ker}_{\mathbb{C}}\left(P_{f}+1\right) \ni v \mapsto \mathcal{Y}(v) \in \mathfrak{g}$ is injective. To show the surjectiveness of this mapping, we take an arbitrary holomorphic vector field $X \in \mathfrak{g}$. Then by Lemma $1.4, X$ is written as $\mathcal{Y}(u)$ for some $u \in C^{\infty}(M)_{\mathbb{C}}$. Since $\bar{\partial} \mathcal{Y}(u)=0$, the formula (3.1.2) shows that

$$
\int_{M}\left(\bar{\partial}\left\{\left(P_{f}+1\right) u\right\}, \bar{\partial} v\right)_{\omega} e^{f} \omega^{m}=0
$$

for all $v \in \mathbb{C}^{\infty}(M)_{\mathbb{C}}$. By setting $v=\left(P_{f}+1\right) u$, we now conclude that $u \in \operatorname{Ker}_{\mathbb{C}}\left(P_{f}+1\right)$, as required.

Q.E.D.

Remark 3.2. In 3.1 above, if we express $X \in \mathfrak{g}$ as $\mathcal{Y}(u)$ for some $u \in \operatorname{Ker} \mathbb{C}\left(P_{f}+1\right)$, the function $u$ coincides with $u_{X}$ in $\S 1$. In fact, from $\mathcal{Y}(u)=\mathcal{Y}\left(u_{X}\right)=X$, we see that $u-u_{X}$ is constant. By Remark 2.5 together with Proposition 3.4 below, we also have

$$
\int_{M} u \omega^{m}=\frac{(2 \pi)^{m+1} \mathcal{F}(X)}{(m+1) \sqrt{-1}}=\int_{M} u_{X} \omega^{m} .
$$

Hence $u=u_{X}$ (cf. [59]). Moreover, if $(M, \omega)$ is an Einstein-Kähler manifold, then we have $P_{f}=\square$ by $f=0$. Therefore, the identity $u_{\mathrm{g}}=$ $\operatorname{Ker}_{\mathbb{C}}\left(\square_{\omega}+1\right)$ in Theorem 1.6 follows from 3.1 , and $u_{\mathfrak{k}}=\operatorname{Ker}_{i \mathbb{R}}\left(\square_{\omega}+1\right)$ can be obtained similarly.

Suppose now that a compact lie group $H$ acts as holomorphic isometries on our Kähler manifold $(M, \omega)$. Let $\mathfrak{h}$ be the Lie algebra of $H$, and let $\mathfrak{k}$ be the space of Killing vector fields on $(M, \omega)$. Then by $(1.3)$, we have $\mathfrak{h} \subset \mathfrak{k} \subset \mathfrak{g}$. Therefore, we always regard $\mathfrak{h}$ as a real Lie subalgebra of $\mathfrak{g}=H^{0}(M, \mathcal{O}(T M))$. Now, by 3.1 and 3.2 above, every $X \in \mathfrak{h}$ is written as $\mathcal{Y}\left(u_{X}\right)$, with $u_{X} \in \operatorname{Ker}_{\mathbb{C}}\left(P_{f}+1\right)$. It is well-known that, by $\mathcal{Y}\left(u_{X}\right) \in \mathfrak{k}$, the real part of $u_{X}$ is constant (see for instance [29; p.37]). In view of

$$
\int_{M} u_{X} e^{f} \omega^{m}=-\ll P_{f} u_{X}, 1 \gg=0 \quad \text { (cf. (3.1.1)), }
$$

the function $u_{X}$ is purely imaginary. We now define $\mu: M \rightarrow \mathfrak{h}^{*}$. by

$$
<\mu(p), X>=(\sqrt{-1})^{-1} u_{X}(p), \quad(p, X) \in M \times \mathfrak{h} .
$$

As in $\S 1$, put $X_{\mathbb{R}}:=X+\bar{X}$. Then $\mu$ satisfies

$$
\begin{aligned}
d<\mu, X> & =i\left(X_{\mathbb{R}}\right) \omega, & & X \in \mathfrak{h}, \\
\mu \circ \sigma & =\operatorname{Ad}\left(\sigma^{-1}\right)^{*} \mu, & & \sigma \in H .
\end{aligned}
$$


In symplectic geometry, a map $\mu: M \rightarrow \mathfrak{h}^{*}$ satisfying (3.3) and (3.4) is called a moment map for the action of $H$ on $M$. We can characterize our $\mu$ by the differential equation

$$
P_{f} \mu+\mu=0
$$

We now endow $\mathfrak{h}^{*}$ with the push-forward $(\mu)_{*}\left(\omega^{m}\right)$ by $\mu$ of the Liouville measure $\omega^{m}$ on $M$ (see [23]).

Proposition 3.5 ([29], [59]). The character $\mathcal{F}$ vanishes if and only if the barycenter $\bar{\mu}$ of the image of the moment map $\mu$ lies at 0 .

Proof. Let $X=\mathcal{Y}\left(u_{X}\right) \in \mathfrak{h}$. Since $\left(P_{f}+1\right) u_{X}=0$, we have

$$
\begin{aligned}
& \sqrt{-1}<\bar{\mu}, X>=\sqrt{-1} \int_{M}<\mu, X>(\mu)_{*}\left(\omega^{m}\right)=\int_{M} u_{X} \omega^{m} \\
& =-\int_{M}\left(\square u_{X}-X f\right) \omega^{m}=-\sqrt{-1}(2 \pi)^{m+1}(m+1)^{-1} \mathcal{F}(X) .
\end{aligned}
$$

Remark 3.6. An m-dimensional compact complex manifold with $c_{1}>0$ and with an almost-homogeneous holomorphic $\left(\mathbb{C}^{*}\right)^{m}$-action is called a toric Fano manifold. As noted in Mabuchi [58], geometric questions of Einstein-Kähler metrics on such manifolds can be reformulated in terms of the geometry of convex bodies, since the image of the corresponding moment maps are convex polytopes in $\mathbb{R}^{m}$. For instance, in [58], Proposition 3.5 is stated as the coincidence of $\mathcal{F}$ and $\bar{\mu}$ up to constant multiple. Similarly, [58] provides another proof, from the viewpoints of symplectic geometry, for the result of Koiso and Sakane [48] on the existence of Einstein-Kähler metrics.

Though the result of Koiso and Sakane will be explained in $\S 6$ in detail, we shall here discuss their result, as an introduction to that section, from the viewpoints of symplectic geometry following [58]; see also [29] in which Mabuchi's proof is treated in the setup of this section. We shall also review the results in [28].

For the coadjoint action of $H$ on $\mathfrak{h}^{*}$, let $H_{c}$ be the isotropy subgroup of $H$ at $c \in \mathfrak{h}^{*}$. By (3.4), $H_{c}$ leaves $\mu^{-1}(c)$ invariant as a set. We now suppose that $c \in \mathfrak{h}^{*}$ is a regular value of $\mu$, and that $H_{c}$ acts freely on $\mu^{-1}(c)$. It is well-known that $M_{c}:=\mu^{-1}(c) / H_{c}$ inherits a unique Kählerian structure described as follows. Let $\pi_{c}: \mu^{-1}(c) \rightarrow M_{c}$ be the natural projection and $\iota_{c}: \mu^{-1}(c) \hookrightarrow M$ the inclusion. The complex structure of $M_{c}$ is induced by the $J$-invariant distribution $\mathcal{D} \subset T \mu^{-1}(c)$ 
obtained as the orthogonal complements of the tangents of the $H_{c}$-orbits. Endow $M_{c}$ with the Kähler form $\omega_{c}$ defined by

$$
\pi_{c}^{*} \omega_{c}=\iota_{c}^{*} \omega
$$

$\left(M_{c}, \omega_{c}\right)$ is called the symplectic quotient (Marsden and Weinstein [61]; see also [36]). Note that $c$ defines naturally a left-invariant 1 -form on $H_{c}$. Hence, via the identification of $H_{c}$ with each $H_{c}$-orbit in $\mu^{-1}(c)$, we can regard $c$ as a 1 -form on $\mu^{-1}(c)$ by setting $\langle c, Y\rangle=0$ for all vectors $Y$ in $\mathcal{D}$. We then have (see $[29 ; \mathrm{p} .112]$ for a proof):

Lemma 3.7 (cf. [23], [28], [58]).

$$
\pi_{c}^{*} \operatorname{Ric}\left(\omega_{c}\right)=\pi_{c}^{*} \omega_{c}+2 \pi d c+\pi_{c}^{*}(\text { exact form }) .
$$

Applying this formula to $c=0$, we obtain

$$
\operatorname{Ric}\left(\omega_{0}\right)=\omega_{0}+\text { exact form, }
$$

since $\pi_{c}$ is surjective. Therefore, $c_{1}\left(M_{0}\right)$ is represented by the positive definite $(1,1)$-form $\omega_{0}$. In particular,

Theorem $3.8([28]) . \quad c_{1}\left(M_{0}\right)>0$.

We shall now give examples of Theorem 3.8.

Example $3.9([28]) . \quad$ (1) Let $M$ be $\left(\mathbb{P}^{1}\right)^{3}=\mathbb{P}^{1}(\mathbb{C}) \times \mathbb{P}^{1}(\mathbb{C}) \times \mathbb{P}^{1}(\mathbb{C})$ and let $H=S^{1}=\left\{e^{\sqrt{-1} \theta} ; \theta \in \mathbb{R}\right\}$ which acts diagonally on $\left(\mathbb{P}^{1}\right)^{3}$ by a common action $S^{1} \times \mathbb{P}^{1}(\mathbb{C}) \ni\left(z_{0}: z_{1}\right) \mapsto\left(z_{0}: e^{\sqrt{-1} \theta} z_{1}\right) \in \mathbb{P}^{1}(\mathbb{C})$. Then the corresponding moment map $\mu:\left(\mathbb{P}^{1}\right)^{3} \rightarrow \mathfrak{h}^{*} \cong \mathbb{R}$ is given by

$$
\begin{aligned}
\mu\left(\left(z_{0}: z_{1}\right),\left(w_{0}: w_{1}\right)\right. & \left.,\left(v_{0}: v_{1}\right)\right) \\
& =\frac{\left|z_{0}\right|^{2}-\left|z_{1}\right|^{2}}{\left|z_{0}\right|^{2}+\left|z_{1}\right|^{2}}+\frac{\left|w_{0}\right|^{2}-\left|w_{1}\right|^{2}}{\left|w_{0}\right|^{2}+\left|w_{1}\right|^{2}}+\frac{\left|v_{0}\right|^{2}-\left|v_{1}\right|^{2}}{\left|v_{0}\right|^{2}+\left|v_{1}\right|^{2}} .
\end{aligned}
$$

The symplectic quotient $\left(M_{0}, \omega_{0}\right)$ in this case is the complex projective space $\mathbb{P}^{2}(\mathbb{C})$ with three non-collinear points blown up. If we take $M=$ $\left(\mathbb{P}^{1}\right)^{5}$ and $K=\mathrm{SO}(3)$, then the symplectic quotient $\left(M_{0}, \omega_{0}\right)$ is the manifold obtained from $\mathbb{P}^{2}(\mathbb{C})$ by blowing up at 4 points in general position.

Suppose for the rest of this chapter that $H=S^{1}=\left\{e^{\sqrt{-1} \theta} ; \theta \in \mathbb{R}\right\}$. Then the complexification $H^{\mathbb{C}}=\mathbb{C}^{*}=\left\{z=r e^{\sqrt{-1} \theta} ; r>0, \theta \in \mathbb{R}\right\}$ acts naturally on $M$. We now put $X=2 \pi \sqrt{-1} \partial / \partial z$ so that $\exp X=$ 
$\operatorname{id}_{M}$. The corresponding real vector field $X_{\mathbb{B}}$ is $2 \pi \partial / \partial \theta \in \mathfrak{h}$, and via the identification of $X$ with $X_{\mathbb{R}}$, we can regard $X$ as an element of $\mathfrak{h}$. Note that $d \theta \in \mathfrak{h}^{*}$ defines a connection form on the $S^{1}$-bundle $\mu^{-1}(c)$ over $M_{c}$. Moreover, we identify $\mathfrak{h}^{*}$ with $\mathbb{R}$ by sending $t \in \mathbb{R}$ to $c:=$ $(2 \pi)^{-1} t d \theta \in \mathfrak{h}^{*}$. We assume further that the interior $\operatorname{int}(I)=(a, b)$ of the image $I=[a, b] \subset \mathbb{R}$ of $M$ under the mapping $\mu$ consists of regular values of $\mu$. Then $M_{c}, c \in \operatorname{int}(I)$, are mutually biholomorphic and the $S^{1}$-bundles $\mu^{-1}(c) \rightarrow M_{c}, c \in \operatorname{int}(I)$, are all isomorphic. By $\Sigma$, we denote the $S^{1}$-bundle: $\mu^{-1}(0) \rightarrow M_{0}$. Integration along fibres together with 3.7 yields the following.

Theorem 3.10 ([58], [48], [71]).

$$
\mathcal{F}(X)=m(m+1) \int_{a}^{b} t\left(c_{1}\left(M_{0}\right)+t c_{1}(\Sigma)\right)^{m-1}\left[M_{0}\right] d t .
$$

We are now in a position to explain the theorem of Koiso and Sakane. For the sake of simplicity, we only state this theorem in the following restricted situation (see $\S 6$ for treatments in more general setup). Let $L$ be a holomorphic line bundle over an Einstein-Kähler manifold $\left(M_{0}, \omega_{0}\right)$. We assume that $L$ carries a Hermitian metric $h$ such that the eigenvalues of $c_{1}(L, h)$ with respect to $\omega_{0}$ are all constant. Let $M$ be the projective bundle $\mathbb{P}\left(\mathcal{O}_{M_{0}} \oplus L\right)$, which is naturally regarded as a compactification of $L$. Then $S^{1}$ acts on $M$ by the scalar multiplication of $\{|z|=1\}$ on $L$, so that we have the above situation if we further assume $c_{1}(M)>0$. The theorem of Koiso and Sakane states that $M$ admits an Eistein-Kähler metric if and only if Futaki's obstruction $\mathcal{F}$ for $M$ vanishes. Note that the first step of the proof of this theorem is to show that the equation for Einstein-Kähler metrics can be reduced to an ordinary differential equation along $I$. Solutions to this equation always exist over $\operatorname{int}(I)$ and define Einstein-Kähler metrics on $\mu^{-1}(\operatorname{int}(I))$. The second step is to show that the condition $\mathcal{F}(X)=0$ implies that a suitably chosen Einstein-Kähler metric on $\mu^{-1}(\operatorname{int}(I))$ extends to $\mu^{-1}(\partial I)$ to define a smooth Einstein-Kähler metric on the whole manifold $M$. In the second step, the expression 3.9 for $\mathcal{F}$ is essentially used. As an application, one can show that the $\mathbb{P}^{1}$-bundle $M$ in 2.9 , for which both obstructions by Matsushima or Futaki vanish, admits an Einstein-Kähler metric.

In Mabuchi $[58 ; \S 10]$, the Einstein-Kähler metric, for instance, in the case where $n_{1}=n_{2}=1$ in 2.9 was described very explicitly. Recall that $M$ is the projective bundle of the 2-plane bundle $E=p_{1}^{*} L_{1} \oplus p_{2}^{*} L_{2} ; M$ may alternatively be expressed as the projective bundle of $\mathcal{O}_{\mathbb{P}^{1} \times \mathbb{P}^{1}} \oplus L$, 
where $L=p_{1}^{*} L_{1}^{-1} \otimes p_{2}^{*} L_{2}$. Note that $E^{0}=E-(0$-section $)$ is described as follows. Consider $\mathbb{C}^{4}=\left\{(x, y) \in \mathbb{C}^{2} \times \mathbb{C}^{2}\right\}$ and $\left(\mathbb{P}^{1}\right)^{2}=\{(u, v) \in$ $\left.\mathbb{P}^{1}(\mathbb{C}) \times \mathbb{P}^{1}(\mathbb{C})\right\}$, where we write $x, y \in \mathbb{C}^{2}$ as $x=\left(x_{1}, x_{2}\right), y=\left(y_{1}, y_{2}\right)$ with $x_{i}, y_{i} \in \mathbb{C}$, and write also $u, v \in \mathbb{P}^{1}(\mathbb{C})$ as $u=\left(u_{1}: u_{2}\right), v=$ $\left(v_{1}: v_{2}\right)$ by homogeneous coordinates. Then

$$
E^{0}=\left\{(x, y, u, v) \in\left(\mathbb{C}^{4}-\{0\}\right) \times\left(\mathbb{P}^{1}\right)^{2} ; x_{1} u_{2}=x_{2} u_{1}, y_{1} v_{2}=y_{2} v_{1}\right\}
$$

Moreover, $\mathbb{C}^{*}$ acts on $E^{0}$ by $\mathbb{C}^{*} \times E^{0} \ni(t,(x, y, u, v)) \mapsto(t x, t y, u, v) \in$ $E^{0}$, and $M$ is nothing but the quotient space $E^{0} / \mathbb{C}^{*}$. Therefore, $L$ is an open dense subset of $M$ defined by

$$
L=\left\{(x, y, u, v) \in E^{0} ; x \neq 0\right\} / \mathbb{C}^{*} .
$$

By the 0 -section and the $\infty$-section of $L$, we respectively mean the subsets $\{y=0\}$ and $\{x=0\}$ in $M$. In terms of the natural Hermitian metric on $L$, the distance $\rho$ from the 0 -section measured along fibres is

$$
\rho=\rho(x, y, u, v)=\|x\|^{-1}\|y\|=\sqrt{\left(\left|y_{1}\right|^{2}+\left|y_{2}\right|^{2}\right) /\left(\left|x_{1}\right|^{2}+\left|x_{2}\right|^{2}\right)}
$$

We now consider the fibrewise action of $S^{1}$ on the line bundle $L$ coming from the multiplication by $S^{1}=\left\{z=e^{\sqrt{-1} \theta} ; \theta \in \mathbb{R}\right\}$. Then by the above expression of $L$, each $z \in S^{1} \operatorname{maps}(x, y, u, v)$ to $(x, z y, u, v)$. Obviously, this $S^{1}$-action extends to a $\mathbb{C}^{*}$-action on $M$. If we put $r=-\log \rho^{2}$, the equation for Einstein-Kähler metrics on $M$ can be written as

$$
\frac{d^{2} \varphi}{d r^{2}}\left(1+\frac{1}{2} \frac{d \varphi}{d r}\right)\left(1-\frac{1}{2} \frac{d \varphi}{d r}\right)=\exp (-\varphi)
$$

We define a real-valued $C^{\infty}$ function $\mu$ on $L$ by

$$
\begin{aligned}
\rho^{2} & =\exp \left(-4 \int_{0}^{\mu} \frac{4-\tau^{2}}{\left(7-\tau^{2}\right)\left(1-\tau^{2}\right)} d \tau\right) \\
& =\frac{1-\mu}{1+\mu}\left(\frac{\sqrt{7}-\mu}{\sqrt{7}+\mu}\right)^{\frac{1}{\sqrt{7}}} .
\end{aligned}
$$

Note that $\mu$ tends to 1 as $\rho \rightarrow 0$ and that $\mu$ tends to -1 as $\rho \rightarrow \infty$. One can see that the function $\mu$ on $L$ extends over the 0 -section and the $\infty$ section to give a $C^{\infty}$ function on $M$. In fact, $\mu: M \rightarrow[-1,1] \subset \mathbb{R}$ turns out to be the moment map for our $S^{1}$-action on the Einstein-Kähler manifold $M$. The desired Einstein-Kähler form on $M$ is expressed as 
$\sqrt{-1} \bar{\partial} \partial \log \Omega$ where

$$
\begin{aligned}
\Omega & =\sqrt{-1} 16 \exp (-\varphi) \omega_{0}^{2} \wedge \partial \rho \wedge \bar{\partial} \rho / \rho^{2} \\
& =\sqrt{-1}\left(7-\mu^{2}\right)\left(1-\mu^{2}\right) \omega_{0}^{2} \wedge \partial \rho \wedge \bar{\partial} \rho / \rho^{2}
\end{aligned}
$$

$\omega_{0}$ being the Einstein-Kähler form of the base space $\mathbb{P}^{1} \times \mathbb{P}^{1}$ defined by

$$
\omega_{0}:=\frac{\sqrt{-1}}{\pi}\left(\partial \bar{\partial} \log \left(\left|u_{1}\right|^{2}+\left|u_{2}\right|^{2}\right)+\partial \bar{\partial} \log \left(\left|v_{1}\right|^{2}+\left|v_{2}\right|^{2}\right)\right) .
$$

We refer to $[58 ; 10.3 .1,10.3 .2]$, to see how one can derive this solution.

\section{$\S 4$. Chern-Simons invariants and the group lifting of $\mathcal{F}$}

In $\S 2$, we showed that $\mathcal{F}$ appears as one of the $G$-invariant polynomials $\mathcal{F}_{\phi}$. These $G$-invariant polynomials are related to the classical invariants such as the equivariant cohomology, the Lefschetz numbers and the Chern-Simons invariants (see e.g. [2], [13], [10], [32], [33], [30]). One of the purposes of this section is to show that $\mathcal{F}$ can be interpreted as the Godbillon-Vey invariant in the foliation theory (cf. [32]). The reason why we take up this topic is because it helps us find how the Lie algebra character $\mathcal{F}: \mathfrak{g} \rightarrow \mathbb{C}$ can be lifted to a group character of $G$ (cf. [14]; see also [27], [57], [30]). Let us begin with a brief review of the theory of Chern, Simons and Cheeger $[20],[19]$.

4.1. Let pr: $P \rightarrow M$ be a principal bundle over a smooth manifold $M$ with structure group $H$, where $H$ is a Lie group. Let $\theta$ be a connection form for $P$, and $\Theta$ the corresponding curvature form. The Chern-Weil theory says that, given an $H$-invariant polynomial $\phi$, the smooth form $\phi(\Theta)$ on $P$ descends to a smooth closed form on $M$. Its de Rham class does not depend on the choice of $\theta$ and is called the characteristic class of $\phi$. However, the smooth form $\phi(\Theta)$ is exact on $P$. More precisely, there is a smooth form $T \phi(\theta)$, defined in a canonical way and called the transgression form, such that

$$
\phi(\Theta)=d T \phi(\theta)
$$

If $\phi(\Theta)=0$ identically, then $T \phi(\theta)$ is a closed form on $P$. Unlike $\phi(\Theta)$, the form $T \phi(\theta)$ does not necessarily descend to a smooth form on $M$. However, if the Weil image $[\phi(\Theta)] \in H^{*}(B H ; \mathbb{R})$ is an integral class, there is an $\mathbb{R} / \mathbb{Z}$-cohomology class $S_{\phi}(\theta)$ on $M$ such that

$$
\operatorname{pr}^{*} S_{\phi}(\theta)=[T \phi(\theta)]^{\prime},
$$


where the right-hand side denotes the modulo $\mathbb{Z}$ reduction of the de Rham class $[T \phi(\theta)]$ (see [20]). The class $S_{\phi}(\theta)$ may not be uniquely determined by $\phi$ and $\theta$. This ambiguity is caused by torsion classes of the integral cohomology of the classifying space $B H$. To be more precise, consider the set $I(H)$ of all $H$-invariant polynomials, and let $\widetilde{\Theta}$ be the curvature form of the universal connection of the universal bundle: $E H \rightarrow B H$, and $H^{*}(B H ; \mathbb{Z}) \ni u \mapsto u_{\mathbb{R}} \in H^{*}(B H ; \mathbb{R})$ the natural map. Take a pair $(\phi, u) \in I(H) \times H^{*}(B H ; \mathbb{Z})$ such that

$$
[\phi(\widetilde{\Theta})]=u_{\mathbb{R}}
$$

We then have a universal object $\widetilde{S}_{\phi, u}$, defined to be a differential character (cf. [19]), with the following property. For every $H$-bundle: $P \rightarrow M$ with an $H$-connection $\theta$ such that $\phi(\Theta)=0$, the associated $\mathbb{R} / \mathbb{Z}$-class on $M$ is just the pull-back $w^{*} \widetilde{S}_{\phi, u}$ of $\widetilde{S}_{\phi, u}$, where $w: M \rightarrow B H$ is the connection-preserving classifying map. We now put

$$
S_{\phi, u}(\theta)=w^{*} \widetilde{S}_{\phi, u},
$$

which is called the Simons class and has functorial properties. Note that, if $H=U(m)$, the cohomology $H^{*}(B H ; \mathbb{Z})$ has no torsion so that the Simons class is determined only by $\phi$. If $G$ is a complex Lie group, the Simons class can be defined as a $\mathbb{C} / \mathbb{Z}$-class (see [29]).

4.2. We now apply the above theory to transversely holomorphic foliations. Let $N$ be a smooth manifold. We denote by $T_{\mathbb{C}} N\left(\right.$ resp. $\left.T_{\mathbb{C}}^{*} N\right)$ the complexified tangent (resp. cotangent) bundle of $N$. A holomorphic foliation $F o l$ of codimension $q$ in $N$ consists of an open covering $\left\{U_{i}\right\}$ of $N$ and submersions $f_{i}: U_{i} \rightarrow V_{i} \subset \mathbb{C}^{q}$ such that if $U_{i} \cap U_{j} \neq \phi$ then

$$
f_{j} \circ f_{j}^{-1}: V_{i} \cap V_{j} \rightarrow V_{i} \cap V_{j}
$$

is biholomorphic. On each $U_{i}$, we choose a system of local coordinates $z_{i}^{1}, \ldots, z_{i}^{q}, x_{i}^{1}, \ldots, x_{i}^{r}$ such that $z_{i}^{\alpha}, 1 \leq \alpha \leq q$, are the complex coordinates induced by the natural ones on $V_{i} \subset \mathbb{C}^{q}$ and that $x_{i}^{\beta}, 1 \leq \beta \leq r$, are the real coordinates in vertical directions. We then have a global subbundle $T_{1,0}^{*}$ of $T_{\mathbb{C}}^{*} N$ spanned by $d z_{i}^{\alpha}, 1 \leq \alpha \leq q$, over each $U_{i}$, and a section of $T_{\mathbb{C}}^{*} N$ is said to be of type $(1,0)$ if it is a section of $T_{1,0}^{*}$. Let $E$ be the subbundle of $T_{\mathbb{C}} N$ annihilated by the covectors in $T_{1,0}^{*}$, and define the normal bundle $\nu(F o l)$ of $F o l$ by

$$
\nu(F o l)=T_{\mathbb{C}} N / E,
$$


which is identified, over each $U_{i}$, with the holomorphic tangent bundle of $V_{i}$. Let $\theta$ be a connection of type $(1,0)$ on $\nu(F o l)$ and $\Theta$ the corresponding curvature form. Since $\operatorname{dim}_{\mathbb{C}} V_{i}=q$, the Chern forms of $\nu(F o l)$ with respect to $\theta$ vanish identically if their degrees exceed $q$ :

$$
c^{\gamma}(\Theta)=c_{1}^{\gamma_{1}} \ldots c_{q}^{\gamma_{q}}(\Theta)=0, \quad|\gamma|=\gamma_{1}+\cdots+\gamma_{q}>q .
$$

This vanishing theorem is due to Bott, and gives us an obstruction for a distribution on a smooth manifold to be integrable when the normal bundle of the distribution admits an almost complex structure. Now, by the Bott vanishing theorem, we have the Simons classes

$$
S_{c^{\gamma}}(\theta) \in H^{2|\gamma|-1}(N ; \mathbb{C} / \mathbb{Z})
$$

for $\gamma=\left(\gamma_{1}, \ldots, \gamma_{q}\right)$ with $|\gamma|>q$. For instance, if $|\gamma|=q+1$, the Simons class $S_{c^{\gamma}}(\theta)$ is independent of the choice of the connection $\theta$. (see [19]). Thus we may write it as $S_{c^{\gamma}}(\mathrm{Fol})$. Furthermore, $S_{c_{1}^{q+1}}(\mathrm{Fol})$ is the so-called Godbillon-Vey class.

4.3. Let $M$ be an m-dimensional compact complex connected manifold and consider the group $G:=\operatorname{Aut}(M)$ of holomorphic automorphisms equipped with discrete topology. Let $E G \rightarrow B G$ be the universal bundle and put $N=E G \times_{G} M$. Then $p: N \rightarrow B G$ is a locally trivial $M$-bundle and the local trivialization induces a holomorphic foliation of codimension $m$ in $N$ with leaves transverse to the fibres. Integrating the Godbillon-Vey class along fibres, we obtain

$$
p_{*} S_{c_{1}^{m+1}}(F o l) \in H^{1}(B G ; \mathbb{C} / \mathbb{Z})
$$

Since $G$ is regarded as a discrete group, $H^{1}(B G ; \mathbb{C} / \mathbb{Z})$ is isomorphic to the additive group of all characters of $G$ into $\mathbb{C} / \mathbb{Z}$. This isomorphism is constructed as follows. Since $\pi_{1}(B G) \cong G$, every $a \in G$ defines a homology class $\bar{a} \in G /[G, G]=H_{1}(B G ; \mathbb{Z})$. Now, to each $\alpha \in H^{1}(B G ; \mathbb{C} / \mathbb{Z})$, we can associate a $\mathbb{C} / \mathbb{Z}$-valued character $\chi_{\alpha}$ of $G$ by $\chi_{\alpha}(a)=\langle\alpha, \bar{a}\rangle$, using the natural pairing $<,>: H^{1}(B G ; \mathbb{C} / \mathbb{Z}) \times H_{1}(B G ; \mathbb{Z}) \rightarrow \mathbb{C} / \mathbb{Z}$.

4.4. We now fix an element $a \in G$ and consider the homomorphism $\iota: \mathbb{Z} \rightarrow G$ defined by $\iota(n)=a^{n}$. Let $\varphi_{\iota}: B \mathbb{Z}\left(=S^{1}\right) \rightarrow B G$ be the corresponding classifying map. Then $\left[\varphi_{\iota}(B \mathbb{Z})\right]=[a]$ as a homology class, and the $M$-bundle $N \mathbb{Z}:=E \mathbb{Z} \times{ }_{\iota} M \rightarrow B \mathbb{Z}$ is induced from $E G \times_{G} M \rightarrow$ $B G$ by $\varphi_{\iota}$. Since $E \mathbb{Z}=\mathbb{R}$, our $N \mathbb{Z}$ is the quotient of $\mathbb{R} \times M$ by the action of $\mathbb{Z}$ defined by

$$
n(s, y)=\left(s+n, a^{n}(y)\right)
$$


where $n \in \mathbb{Z}, s \in \mathbb{R}$ and $y \in M$. The natural holomorphic foliation of codimension $m$ in $N \mathbb{Z}$ induced by the local trivialization of the $M$ bundle: $N \mathbb{Z} \rightarrow B \mathbb{Z}$ is called the suspension foliation, the leaves of which are real 1-dimensional.

Choose an arbitrary holomorphic vector field $X$ on $M$. Recall that $X_{\mathbb{R}}=X+\bar{X}$. For each $t \in \mathbb{R}$, we take $a=a(t)$ to be $\exp \left(t X_{\mathbb{R}}\right)$, and let $p_{t}: N \mathbb{Z} \rightarrow B \mathbb{Z}$ be the associated $M$-bundle. Consider the resulting suspension foliation $\mathrm{Fol}_{t}$. Then by [19; Proposition 2.9], one obtains

Theorem 4.5 (Futaki and Morita [32]).

$$
\mathcal{F}(X)=\frac{d}{d t}\left\{\left(p_{t}\right)_{*} S_{c_{1}^{m+1}}\left(F_{o l}\right)\left[S^{1}\right]\right\}=\left(p_{t}\right)_{*} S_{c_{1}^{m+1}}\left(F o l_{t}\right)\left[S^{1}\right]_{\mid t=1} .
$$

Thus, to see how the Lie algebra character $\mathcal{F}: \mathfrak{g} \rightarrow \mathbb{C}$ lifts to a group character $\widehat{\mathcal{F}}: G \rightarrow \mathbb{C} / \ell \mathbb{Z}$, it suffices to compute the right-hand side of Theorem 4.5 for any given $a \in G$. Note here that $\ell$ is a suitable positive integer, and we may choose for instance $\ell=1$. It seems, however, difficult to compute the right-hand side of Theorem 4.5 naively since it amounts to giving the connection-preserving classifying map explicitly. We use, instead, the theory of exotic characteristic classes of foliations by Bott [13] to derive an explicit formula for the imaginary part of $\widehat{\mathcal{F}}$. To explain this theory, let $W U(q)$ be the differential graded algebra

$$
\begin{aligned}
W U(q)=\mathbb{C}\left[c_{1}, \ldots, c_{q}\right] /\{\operatorname{deg}>2 q\} & \oplus \mathbb{C}\left[\bar{c}_{1}, \ldots, \bar{c}_{q}\right] /\{\operatorname{deg}>2 q\} \\
& \oplus \Lambda\left(u_{1}, \ldots, u_{q}\right) /\{\operatorname{deg}>q\},
\end{aligned}
$$

where $\operatorname{deg} c_{i}=\operatorname{deg} \bar{c}_{i}=2 i, \operatorname{deg} u_{i}=1$ and

$$
d c_{i}=d \bar{c}_{i}=0, \quad d u_{i}=c_{i}-\bar{c}_{i} .
$$

As in 4.4, we fix $a \in G$ and consider the $M$-bundle: $N \mathbb{Z} \rightarrow B \mathbb{Z}=$ $S^{1}$. Let $\mathrm{Fol}$ be the corresponding suspension foliation. For $\nu(\mathrm{Fol})$ over $N \mathbb{Z}$, we take a connection $\nabla^{1}$ of type $(1,0)$ (cf. 4.2) and also a Hermitian connection $\nabla^{0}$. Let $\mathrm{pr}_{1}: N \mathbb{Z} \times[0,1] \rightarrow N \mathbb{Z}$ be the natural projection. Then $\nabla:=(1-t) \nabla^{0}+t \nabla^{1}$ defines a connection on the bundle $\operatorname{pr}_{1}^{*} \nu(F o l) \rightarrow N \mathbb{Z} \times[0,1]$. We denote by $\Theta^{0,1}$ the curvature form of this connection. Let $\Lambda(N \mathbb{Z})$ be the set of all smooth forms on $N \mathbb{Z}$. We now define a map $\lambda: W U(q) \rightarrow \Lambda(N \mathbb{Z})$ by

$$
\begin{gathered}
\lambda\left(c_{i}\right)=c_{i}\left(\nabla^{1}\right), \quad \lambda\left(\bar{c}_{i}\right)=\overline{c_{i}\left(\nabla^{1}\right)} \\
\lambda\left(u_{i}\right)=h_{i}-\bar{h}_{i}, \quad \text { where } \quad h_{i}=\int_{0}^{1} c_{i}\left(\Theta^{0,1}\right) .
\end{gathered}
$$


Then $d h_{i}=c_{i}\left(\nabla^{1}\right)-c_{i}\left(\nabla^{0}\right)$. Since $\nabla^{0}$ is Hermitian, $c_{i}\left(\nabla^{0}\right)$ is real. Hence, $d\left(\lambda\left(u_{i}\right)\right)=\lambda\left(d u_{i}\right)$. We therfore have a well-defined induced map

$$
H(\lambda): H^{*}(W U(q)) \rightarrow H^{*}(N \mathbb{Z} ; \mathbb{C})
$$

The map $H(\lambda)$ is independent of the choice of $\nabla^{0}$ and $\nabla^{1}$. Furthermore, by applying the Stokes theorem, we obtain (see $[29 ; \mathrm{p} .92]$ ):

$$
\sqrt{-1} \operatorname{Im} S_{c_{1}^{m+1}}(F o l)=\frac{1}{2} \sum_{k=0}^{m} H(\lambda)\left(c_{1}^{k} u_{1} \bar{c}_{1}^{m-k}\right) .
$$

Given a Hermitian metric for (the tagent bundle of) $M$, one can compute the right-hand side by giving $\nabla^{0}$ and $\nabla^{1}$ explicitly. This together with Theorem 4.5 yields the following formula of Futaki:

Theorem $4.6([27])$. Let $\sqrt{-1} \operatorname{Im} \widehat{\mathcal{F}}$ be the imaginary part of $\widehat{\mathcal{F}}$. Then for all $a \in G$,

$$
\operatorname{Im} \widehat{\mathcal{F}}(a)=\int_{M} \frac{\log \left\{\operatorname{det}\left(a^{*} \eta\right) / \operatorname{det}(\eta)\right\}}{4 \pi} \sum_{k=0}^{m} c_{1}^{k}\left(M ; a^{*} \eta\right) \wedge c_{1}^{m-k}(M ; \eta),
$$

where $\eta$ is an arbitrary Hermitian metric of $M$ and $c_{1}(M ; \eta)$ denotes the first Chern form of $M$ with respect to $\eta$.

4.7. Inspired by the work of Donaldson [22] in which the secondary invariants are used as functionals to find Einstein-Hermitian metrics on stable vector bundles, Mabuchi [57] also found a formula for $\operatorname{Im} \widehat{\mathcal{F}}$ independently. Let $M$ be an $m$-dimensional compact complex connected manifold with $c_{1}(M)>0$. Recall that $\mathcal{K}$ is the set of all Kähler forms which represent $c_{1}(M)$. Choose two Kähler forms $\omega$ and $\omega^{\prime}$ in $\mathcal{K}$. Let $\left\{\omega_{s}=\omega+\sqrt{-1} \partial \bar{\partial} \varphi_{s} ; 0 \leq s \leq 1\right\}$ be a smooth path in $\mathcal{K}$ joining $\omega_{0}=$ $\omega$ and $\omega_{1}=\omega^{\prime}$. We define a functional $\Xi: \mathcal{K} \times \mathcal{K} \rightarrow \mathbb{R}$ by

$$
\Xi\left(\omega, \omega^{\prime}\right)=\int_{0}^{1} \frac{(m+1) d s}{(2 \pi)^{m+1}} \int_{M} \frac{d \varphi_{s}}{d s}\left(\omega_{s}-\operatorname{Ric}\left(\omega_{s}\right)\right) \wedge m \omega_{s}^{m-1} .
$$

Lemma 4.8 ([57]; see also [8]). $\Xi\left(\omega, \omega^{\prime}\right)$ depends only on $\omega, \omega^{\prime}$ and is independent of the choice of the smooth path $\left\{\omega_{s} ; 0 \leq s \leq 1\right\}$ in $\mathcal{K}$. Moreover, $\Xi$ satisfies the 1-cocycle conditions:

$$
\begin{gathered}
\Xi\left(\omega^{\prime}, \omega\right)=-\Xi\left(\omega, \omega^{\prime}\right), \\
\Xi\left(\omega, \omega^{\prime}\right)+\Xi\left(\omega^{\prime}, \omega^{\prime \prime}\right)+\Xi\left(\omega^{\prime \prime}, \omega\right)=0,
\end{gathered}
$$

where $\omega, \omega^{\prime}, \omega^{\prime \prime}$ are arbitrary elements of $\mathcal{K}$.

Let $\omega_{0} \in \mathcal{K}$ be a reference metric. We then define the $\mathcal{K}$-energy map $\mu: \mathcal{K} \rightarrow \mathbb{R}$ by $\mu(\omega)=\Xi\left(\omega_{0}, \omega\right)$. This $\mu$ satisfies: 
Theorem $4.9([57])$. Let $\mathcal{E}$ be the subset of $\mathcal{K}$ consisting of all Einstein-Kähler forms in the class. Then

(1) $\omega$ is a critical point of $\mu$ if and only if $\omega \in \mathcal{E}$.

(2) For every $X \in \mathfrak{g}$, we have

$$
\frac{d}{d t} \mu\left(\exp \left(t X_{\mathbb{R}}\right)^{*} \omega\right)=2 \operatorname{Im} \mathcal{F}(X) .
$$

Let us now endow $\mathcal{K}$ with the natural Riemannian structure defined by

$$
(\varphi, \psi)=\int_{M} \varphi \psi \omega^{m}, \quad \varphi, \psi \in T_{\omega} \mathcal{K}
$$

where we identify $T_{\omega} \mathcal{K}$ and $\left\{\varphi \in C^{\infty}(M)_{\mathbb{R}} ; \int_{M} \varphi \omega^{m}=0\right\}$. In terms of this Riemannian structure, $\mu$ is a convex function (cf. [60]). Then, $\mu$ takes the absolute minimum exactly on $\mathcal{E}$ (cf. [7], [6]), Moreover, Bando and Mabuchi [7] showed that $\mathcal{E}$ is connected, which will be explained in the next section. As in [57], we define $\chi: G \rightarrow \mathbb{R}_{+}$by

$$
\chi(a)=\exp \left\{\Xi\left(\omega_{0}, a^{*} \omega_{0}\right)\right\}, \quad a \in G .
$$

Then $\chi$ becomes a multiplicative character in view of the 1-cocycle conditions satisfied by the functional $\Xi$. To relate $(4.10)$ to Theorem 4.6 , let $\eta \in \mathcal{K}$ be Yau's solution of the equation $\omega_{0}=\operatorname{Ric}(\eta)$. Rewriting (4.10) in terms of $\eta$, one sees that $\log \chi$ coincides with $\operatorname{Im} \widehat{\mathcal{F}}$ up to constant multiple.

Unlike the case of $\operatorname{Im} \mathcal{F}$, the real part $\operatorname{Re} \widehat{\mathcal{F}}: G \rightarrow \mathbb{R} / \ell \mathbb{Z}$ of $\widehat{\mathcal{F}}: G \rightarrow$ $\mathbb{C} / \ell \mathbb{Z}$ cannot be written in a simple and explicit way. However, in view of the identification

$$
\mathbb{C} / \ell \mathbb{Z} \cong \mathbb{C}^{*}, \quad z \leftrightarrow \exp \left(\frac{2 \pi \ell^{-1} z}{\sqrt{-1}}\right)
$$

the following theorem shows that $\widehat{\mathcal{F}}$ can be regarded as the multiplicative character $\operatorname{det}(\rho): G \rightarrow \mathbb{C}^{*}$ below.

Theorem 4.11 ([30], [59]). Let $M$ be an $m$-dimensional compact complex connected manifold with $c_{1}(M)>0$. Take $\ell$ to be 2 or 1 according as $m$ is even or odd. Then there is an algebraic group homomorphism $\rho: G \rightarrow \mathrm{GL}_{\mathbb{C}}(V)$ such that 
(1) $\exp \left(2 \pi \ell^{-1} \operatorname{Im} \widehat{\mathcal{F}}\right)=|\operatorname{det}(\rho)|$

(2) $V$ is a finite dimensional $\mathbb{C}$-vector space of the form

$$
V=\bigoplus_{\nu} m_{\nu} H^{0}\left(M, K_{M}^{-\nu}\right)
$$

where the integers $m_{\nu}$ are nonzero only for finitely many natural numbers $\nu$ and depend only on $m$ and $\nu$.

Note that, for a $\mathbb{C}$-vector space $W$ endowed with a $G$-action, the space $n W($ resp. $-n W)$ denotes the direct sum of $n$-copies of $W$ (resp. $W^{*}$ ) on which $G$ acts naturally.

Example 4.11.1. If $m=2$, then $V=H^{0}\left(M, K_{M}^{-1}\right)$. Moreover, if $m=3$, we have $V=H^{0}\left(M, K_{M}^{-2}\right) \oplus 3 H^{0}\left(M, K_{M}^{-1}\right)^{*}$. As far as the examples of Einstein-Kähler surfaces studied in [30] concerned, the values of $\operatorname{det}(\rho)$ are \pm 1 .

We now consider the most basic case where $\ell=1$ for all $m$, so that $\widehat{\mathcal{F}}$ maps $G$ to $\mathbb{C} / \mathbb{Z}$. Note that $\operatorname{Im} \widehat{\mathcal{F}}$ vanishes if $M$ admits an Einstein-Kähler metric (see [27]). However, it is unclear whether $\operatorname{Re} \widehat{\mathcal{F}}$ says something about the existence of an Einstein-Kähler metric. Recall the following conjecture of Calabi: on a compact complex manifold with $c_{1}(M)>$ 0 and with no non-zero holomorphic vector fields, there will exist an Einstein-Kähler metric. In view of this conjecture, it is of independent interest to find an example of a compact complex manifold with discrete $G$ and with $\operatorname{Re} \widehat{\mathcal{F}} \neq 0$. In [34], by relating $\operatorname{Re} \widehat{\mathcal{F}}$ to the eta invariant for the signature operator acting on certain vector-bundle-valued smooth forms, it is shown that there does exist such an example, though the first Chern class of the manifold is not positive.

\section{$\S 5$. The uniqueness theorem}

In this section, we fix once for all an $m$-dimensional compact complex connected manifold $M$ which admits an Einstein-Kähler metric of positive scalar curvature. Let $\mathcal{K}$ be the set of all Kähler forms on $M$ in the de Rham cohomology class $2 \pi c_{1}(M)_{\mathbb{R}}$, and consider its subset $\mathcal{E}$ consisting of all Einstein-Kähler forms. We then have

$$
\mathcal{E}=\{\omega \in \mathcal{K} ; \operatorname{Ric}(\omega)=\omega\} \neq \phi
$$

and $\mathcal{E}$ is regarded as the moduli space of Einstein-Kähler metrics on $M$. Note that the identity component $G^{0}$ of $G=\operatorname{Aut}(M)$ acts on $\mathcal{E}$ from 
the right by $\mathcal{E} \times G^{0} \ni(\omega, g) \mapsto g^{*} \omega \in \mathcal{E}$. Now, the main purpose of this section is to give a quick review of the following theorem of Bando and Mabuchi [7] with emphasis on its theoretical aspects rather than its technical details:

Theorem 5.1. $\mathcal{E}$ consists of a single $G^{0}$-orbit.

Recall that, for the special case where $M$ is a Kähler C-space, this theorem was first shown by Matsushima [64], though his proof essentially depends on the transitiveness of the $G^{0}$-action on $M$.

We shall now give a couple of consequences of Theorem 5.1. Let $\omega$ be an element of $\mathcal{E}$, and $K$ the corresponding group of the isometries $\left\{g \in G^{0} ; g^{*} \omega=\omega\right\}$ sitting in $G^{0}$. Moreover, denote by $\operatorname{Ker}_{\mathbb{R}}\left(\square_{\omega}+1\right)$ the space of all $v$ in $C^{\infty}(M)_{\mathbb{R}}$ such that $\left(\square_{\omega}+1\right) v=0$. Then by using the notation in $\S 1$, we obtain from Theorem 1.6 the isomorphisms

$$
\begin{aligned}
& T_{\omega}(\mathcal{E}) \cong \mathfrak{g} / \mathfrak{k} \cong \operatorname{Ker}_{\mathbb{R}}\left(\square_{\omega}+1\right), \\
& \mathcal{Y}(v)_{\mathbb{R}} \cdot \omega \leftrightarrow \mathcal{Y}(v) \leftrightarrow v,
\end{aligned}
$$

where we regard $\mathcal{Y}(v)_{\mathbb{B}} \cdot \omega(=-2 \sqrt{-1} \partial \bar{\partial} v)$ as the tangent vector at $t=0$ of the curve $\left\{\exp \left(t \mathcal{Y}(v)_{\mathbb{R}}\right)^{*} \omega ; t \in \mathbb{R}\right\}$ in $\mathcal{E}$. Then the $L^{2}$ inner product

$$
\left(v_{1}, v_{2}\right)_{L^{2}, \omega}:=\int_{M} v_{1} v_{2} \omega^{m} / m !, \quad v_{1}, v_{2} \in \operatorname{Ker}_{\mathbb{P}}\left(\square_{\omega}+1\right),
$$

induces a natural Riemannian structure on $\mathcal{E}$ via the identification of $T_{\omega}(\mathcal{E})$ with $\operatorname{Ker}_{\mathbb{R}}\left(\square_{\omega}+1\right)$. Moreover, for every $g \in G$,

$$
\left(g^{*} v_{1}, g^{*} v_{2}\right)_{L^{2}, g^{*} \omega}=\left(v_{1}, v_{2}\right)_{L^{2}, \omega} \quad v_{1}, v_{2} \in \operatorname{Ker}_{\mathbb{R}}\left(\square_{\omega}+1\right) .
$$

Hence, $G$ acts isometrically on $\mathcal{E}$. Now, note that $G^{0} / K$ has a structure of a Riemannian symmetric space, though the choice of its metric is not unique (even up to constant multiple) if the symmetric space $G^{0} / K$ is reducible. Thus, we obtain:

Corollary 5.1.1. $\mathcal{E}$ is $G^{0}$-equivariantly isometric to the Riemannian symmetric space $G^{0} / K$ endowed with a suitable metric, and furthermore, $G$ acts isometrically on $\mathcal{E}$.

Since $G^{0} / K$ is a Riemannian symmetric space without compact factors, $\mathcal{E}$ is a simply connected Riemannian manifold with nonpositive sectional curvature, so that any compact group acting isometrically on $\mathcal{E}$ has a fixed point. This in particular implies: 
Corollary 5.1.2. For any (possibly non-connected) compact subgroup of $G$, our $M$ always carries an Einstein-Kähler metric invariant under the action of the compact group.

We shall now give a rough idea of how Theorem 5.1 can be proven. Choose a Kähler form $\theta$ in the Kähler class $\mathcal{K}$. Then we start with the following one-parameter family of differential equations (cf. Aubin [4]):

$$
\operatorname{Ric}(\omega)=(1-t) \theta+t \omega, \quad 0 \leq t \leq 1,
$$

where for each $t$, any solution $\omega=\omega_{t}$ for (5.2) is required to sit in $\mathcal{K}$. Note that, by Yau's celebrated theorem [77] on Calabi's conjecture, (5.2) has a unique solution $\omega=\omega_{0}$ at $t=0$. Moreover, $\omega=\omega_{1}$ is a solution for (5.2) at $t=1$ if and only if $\omega_{1} \in \mathcal{E}$. Let $I$ be the unit interval $\{0 \leq t \leq 1\}$. We can now visualize the situation by taking the graph

$$
\Gamma_{\theta}=\{(\omega, t) \in \mathcal{K} \times I ; \operatorname{Ric}(\omega)=(1-t) \theta+t \omega\}
$$

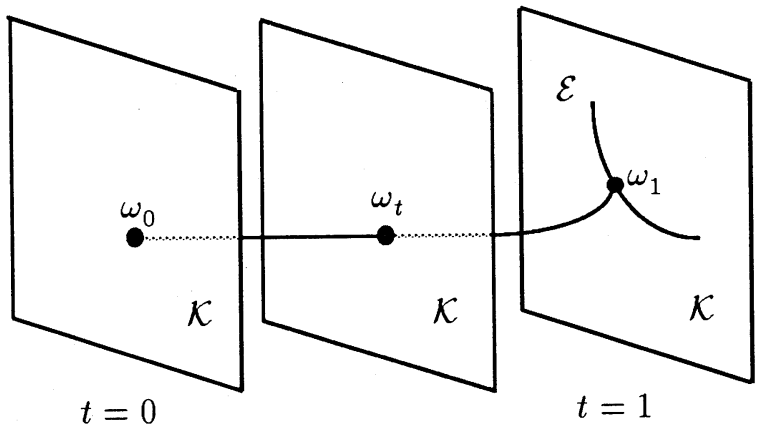

Fig. 1. Figure of Graph $\Gamma_{\theta}$

of the solutions of (5.2). Then the proof of Theorem 5.1 is reduced to showing the following:

Theorem 5.3. Given a $G^{0}$-orbit $\mathbf{O}$ in $\mathcal{E}$, by taking $\theta \in \mathcal{K}$ sufficiently general, we can connect $\mathrm{O}$ with $\omega_{0}$ by a smooth one-parameter family of solutions $\mathcal{F}=\left\{\omega_{t} ; 0 \leq t \leq 1\right\}$ for (5.2) such that

(1) $\omega_{1} \in \mathbf{O}$;

(2) $\Gamma_{\theta} \cap\{t \neq 1\}$ coincides with $\left\{\left(\omega_{t}, t\right) ; 0 \leq t<1\right\}$. 
In fact, for $G^{0}$-orbits $\mathrm{O}_{1}, \mathbf{O}_{2}$ in $\mathcal{E}$, a sufficiently general choice of $\theta$ in $\mathcal{K}$ allows us to connect $\mathrm{O}_{1}, \mathrm{O}_{2}$ with $\omega_{0}$ by families $\mathcal{F}_{1}, \mathcal{F}_{2}$ as above, respectively, and by (2), these families must coincide, since $\Gamma_{\theta}$ is independent of the choice of such $G^{0}$-orbits. Thus, $\mathbf{O}_{1}=\mathbf{O}_{2}$, as required.

Before getting into the study of Theorem 5.3, we shall introduce more notation. Take an $\alpha$ with $0<\alpha<1$, and let $\tilde{\mathcal{K}}, \widetilde{\mathcal{K}}^{2, \alpha}$ be the set of all $\varphi$ in $C^{\infty}(M)_{\mathbb{R}}, C^{2, \alpha}(M)$, respectively, such that $\theta+\sqrt{-1} \partial \bar{\partial} \varphi$ is positive definite everywhere on $M$. Put $V:=\int_{M} \theta^{m}$. Now, we define functionals $\lambda$ and $\iota$ on $\widetilde{\mathcal{K}}^{2, \alpha}$ by setting (cf. [4], [57], [7])

$$
\begin{aligned}
\iota(\varphi):=\lambda(\varphi)-\int_{M} \varphi(\theta+\sqrt{-1} \partial \bar{\partial} \varphi)^{m} / V, & \varphi \in \tilde{\mathcal{K}}^{2, \alpha}, \\
\lambda(\psi):=\int_{0}^{1}\left(\int_{M} \psi(\theta+s \sqrt{-1} \partial \bar{\partial} \psi)^{m} / V\right) d s, & \psi \in \tilde{\mathcal{K}}^{2, \alpha} .
\end{aligned}
$$

Then $\lambda(\psi+C)=\lambda(\psi)+C$ and $\iota(\psi+C)=\iota(\psi) \geq 0$ for all $(\psi, C)$ in $\widetilde{\mathcal{K}}^{2, \alpha} \times \mathbb{R}$. Moreover, for any smooth path $\left\{\psi_{t} ; a \leq t \leq b\right\}$ in $\widetilde{\mathcal{K}}^{2, \alpha}$, one can easily check that

$$
\begin{aligned}
& \frac{d \iota\left(\psi_{t}\right)}{d t}=-\frac{1}{V} \int_{M} \psi_{t}\left(\square_{t} \dot{\psi}_{t}\right)\left(\theta+\sqrt{-1} \partial \bar{\partial} \psi_{t}\right)^{m} \\
& \frac{d \lambda\left(\psi_{t}\right)}{d t}=\frac{1}{V} \int_{M} \dot{\psi}_{t}\left(\theta+\sqrt{-1} \partial \bar{\partial} \psi_{t}\right)^{m}
\end{aligned}
$$

where $\dot{\psi}_{t}:=\partial \psi_{t} / \partial t$, and $\square_{t}$ denotes the Laplacian associated with the Kähler form $\theta+\sqrt{-1} \partial \bar{\partial} \varphi_{t}$.

We shall next give an outline of the proof of Theorem 5.3. The main point of our proof is to solve the equation (5.2) backward in $t$ by starting off with $t=1$ and ending up at $t=0$. Define $\Phi: \widetilde{\mathcal{K}}^{2, \alpha} \times I \rightarrow C^{0, \alpha}(M)_{\mathbb{R}}$, $\Psi: \widetilde{\mathcal{K}}^{2, \alpha} \times I \rightarrow C^{0, \alpha}(M)_{\mathbb{R}}$ by sending elements $(\varphi, t),(\psi, t)$ of $\tilde{\mathcal{K}}^{2, \alpha} \times I$ respectively to

$$
\begin{aligned}
& \Phi(\varphi, t)=\log \left((\theta+\sqrt{-1} \partial \bar{\partial} \varphi)^{m} / \theta^{m}\right)+t \varphi-f \\
& \Psi(\psi, t)=\Phi(\psi, t)+\lambda(\psi)
\end{aligned}
$$

where $f \in C^{\infty}(M)_{\mathbb{R}}$ is the function uniquely determined by

(1) $\operatorname{Ric}(\theta)=\theta+\sqrt{-1} \partial \bar{\partial} f ;$

(2) $\int_{M} \exp (f) \theta^{m}=V$. 
Then one may rewrite the equation (5.2) above in the following more suitable forms:

$$
\begin{array}{ll}
\Phi(\varphi, t)=0, & 0<t \leq 1 \\
\Psi(\psi, t)=0, & 0 \leq t \leq 1 .
\end{array}
$$

Note that, if $\sqrt{-1} \partial \partial$ operates on both sides of $(5.2 \mathrm{a})$ and $(5.2 \mathrm{~b})$, we obtain (5.2) by setting $\omega=\theta+\sqrt{-1} \partial \bar{\partial} \varphi=\theta+\sqrt{-1} \partial \bar{\partial} \psi$. Moreover, for any $(\varphi, t)(\operatorname{resp} .(\psi, t))$ in $\tilde{\mathcal{K}}^{2, \alpha} \times I$ satisfying $(5.2 \mathrm{a})$ (resp. $\left.(5.2 \mathrm{~b})\right)$, the well-known regularity theorem says that $\varphi$ (resp. $\psi$ ) is always in $\widetilde{\mathcal{K}}$. Hence, at each $t \in I-\{0\}$, we have the isomorphism

$$
\begin{aligned}
\{\varphi \in \tilde{\mathcal{K}} ; \Phi(\varphi, t)=0\} & \cong\{\omega \in \mathcal{K} ; \operatorname{Ric}(\omega)=(1-t) \theta+t \omega\}, \\
\varphi & \leftrightarrow \theta+\sqrt{-1} \partial \bar{\partial} \varphi,
\end{aligned}
$$

which allows us to identify $\Gamma_{\theta}$ above with $\Gamma_{\Phi}$ for $0<t \leq 1$, and similarly to identify the whole $\Gamma_{\theta}$ with $\Gamma_{\Psi}$, where

$$
\begin{aligned}
& \Gamma_{\Phi}:=\left\{(\varphi, t) \in \widetilde{\mathcal{K}}^{2, \alpha} \times I ; \Phi(\varphi, t)=0\right\}, \\
& \Gamma_{\Psi}:=\left\{(\psi, t) \in \widetilde{\mathcal{K}}^{2, \alpha} \times I ; \Psi(\psi, t)=0\right\} .
\end{aligned}
$$

Note that there exists just one element $\psi_{0}$ of $\widetilde{\mathcal{K}}$ such that $\Psi\left(\psi_{0}, 0\right)=0$, since $\psi_{0}$ is uniquely characterized by the identities

$$
\omega_{0}=\theta+\sqrt{-1} \partial \bar{\partial} \psi_{0} \text { and } \lambda\left(\psi_{0}\right)=0 \quad \text { (cf. [7]). }
$$

Now, for better understanding of our arguments, we introduce an algebraic equation analogous to (5.2) (and also to (5.2a), (5.2b)) as follows. Let $H: \mathbb{R}^{2} \times I \rightarrow \mathbb{R}^{2}$ be the mapping defined by

$$
H(z, t)=\left(\left(x^{2}+y-1\right)\left(x+x^{2}+y-t\right), x^{2}+y-t^{2}\right)
$$

for $z=(x, y) \in \mathbb{R}^{2}$ and $t \in I$. We then consider the equation:

$$
H(z, t)=0 .
$$

If $H\left(z_{0}, t_{0}\right)=0$ with $t_{0} \neq 1$, then the Jacobian matrix at $z_{0}$ of the mapping $\mathbb{R}^{2} \ni z \mapsto H\left(z, t_{0}\right) \in \mathbb{R}^{2}$ is invertible, and hence the implicit function theorem says that there exists a unique smooth curve $z(t)$ through $z\left(t_{0}\right)=z_{0}$ satisfying $H(z(t), t)=0$ for $(z(t), t)$ in a small neighbourhood of $\left(z_{0}, t_{0}\right)$. (Of course, we can explicitly solve the equation (5.4) and the graph $\Gamma_{H}:=\{(z, t) ; H(z, t)=0\}$ consists of the curves $\left\{\left(\left(t-t^{2}, 2 t^{3}-t^{4}\right), t\right) ; t \in I\right\}$ and $\left\{((x, y), 1) ; y=1-x^{2}\right\}$, 
though for our original equation (5.2), we are far from obtaining an explicit description of $\Gamma_{\theta}$.) We shall now show that the implicit function theorem is applicable also to (5.2) (or more precisely, to $(5.2 \mathrm{a})$ or $(5.2 \mathrm{~b})$ ):

Case 1 (cf. Aubin [4]). Let $\Phi\left(\varphi_{t_{0}}, t_{0}\right)=0$ with $0<t_{0}<1$. For simplicity, we put $\gamma_{0}:=\left(\varphi_{t_{0}}, t_{0}\right)$ and $\theta_{0}:=\theta+\sqrt{-1} \partial \bar{\partial} \varphi_{t_{0}}$. Then, at $\gamma_{0}$, the Fréchet derivative $D_{\varphi} \Phi: C^{2, \alpha}(M)_{\mathbb{R}} \rightarrow C_{\mathbb{R}}^{0, \alpha}$ of $\Phi$ with respect to $\varphi$ is given by

$$
D_{\varphi} \Phi_{\mid \gamma_{0}}(\eta)=\frac{d}{d \varepsilon} \Phi\left(\varphi_{t_{0}}+\varepsilon \eta, t_{0}\right)_{\mid \varepsilon=0}=\left(\square_{\theta_{0}}+t_{0}\right) \eta, \quad \eta \in C^{2, \alpha}(M)_{\mathbb{R}} .
$$

Since $\operatorname{Ric}\left(\theta_{0}\right)-t_{0} \theta_{0}=\left(1-t_{0}\right) \theta$ is positive definite, a theorem of Lichnerowicz [52] asserts that $t_{0}$ is less than the first (positive) eigen value of $-\square_{\theta_{0}}$. Hence $D_{\varphi} \Phi_{\mid \gamma_{0}}$ is invertible.

Case 2 (cf. [7]). Let $t_{0}=0$. Then, in view of the identities $\Psi\left(\psi_{0}, 0\right)=0$ and $\omega_{0}:=\theta+\sqrt{-1} \partial \bar{\partial} \psi_{0}$, it follows that, at $\left(\psi_{0}, 0\right)$, the Fréchet derivative $D_{\psi} \Psi$ of $\Psi$ with respect to $\psi$ is written in the form

$$
C^{2, \alpha}(M)_{\mathbb{R}} \ni \eta \mapsto \square_{\omega_{0}} \eta+\int_{M} \eta \omega_{0}^{m} / V \in C^{0, \alpha}(M)_{\mathbb{R}},
$$

which is invertible.

Thus, we obtained openness for the equation (5.2) for $0 \leq t<1$, and routine works using Ascoli's theorem show that any solution $\omega=\omega_{t_{1}}$ for (5.2) at $t=t_{1}\left(0 \leq t_{1}<1\right)$ extends uniquely to a smooth one-parameter family of solutions $\left\{\omega_{t} ; 0 \leq t \leq t_{1}\right\}\left(=\Gamma_{\theta} \cap\left\{0 \leq t \leq t_{1}\right\}\right)$ for (5.2), provided that we have the following a priori estimates:

Theorem 5.5. Let $\left\{\psi_{t} ; t_{0}<t \leq t_{1}\right\}$ be a smooth one-parameter family of solutions for $(5.2 \mathrm{~b})$ (so that $\Psi\left(\psi_{t}, t\right)=0$ for all $t$ ), where $0 \leq$ $t_{0}<t_{1}<1$. Then there exist positive constants $K_{0}, K_{1}, K_{2}$ independent of $t$ such that we have $\left\|\psi_{t}\right\|_{C^{2, \alpha}} \leq K_{0}$ and $K_{1} \theta \leq \theta+\sqrt{-1} \partial \bar{\partial} \psi_{t} \leq K_{2} \theta$ for all $t$.

Hence, the proof of Theorem 5.3 is reduced to showing Theorem 5.5 plus the following:

Theorem 5.6. For every $G^{0}$-orbit $\mathrm{O}$ in $\mathcal{E}$, taking $\theta$ sufficiently general, we have an $0<\varepsilon \ll 1$ and a smooth one-parameter family of solutions $\left\{\varphi_{t} ; 1-\varepsilon<t \leq 1\right\}$ for $(5.2 \mathrm{a})$ such that $\theta+\sqrt{-1} \partial \bar{\partial} \varphi_{1} \in \mathbf{O}$.

Proof of Theorem 5.5. Note that, by standard arguments (cf. [77], $[3])$, it suffices to find a constant $K$ such that $\left\|\psi_{t}\right\|_{C^{0}} \leq K$ for all $t$, 
where in this proof, constants mean positive real numbers independent of $t$. The proof is divided into three steps.

Step 1. Let $D_{t}$ be the diameter of the Kähler manifold $\left(M, \omega_{t}\right)$, where $\omega_{t}:=\theta+\sqrt{-1} \partial \bar{\partial} \psi_{t}$. Then, for the infimum $-A_{t}$ of the Green function of the Laplacian $\square_{\omega_{t}}$, we have $A_{t} \leq \gamma_{m} D_{t}^{2} / V$ for some constant $\gamma_{m}$ depending on $m$ alone (cf. [7]). Moreover, by $\operatorname{Ric}\left(\omega_{t}\right) \geq t \omega_{t}$, Myers's theorem asserts that $D_{t} \leq \pi \sqrt{(m-1) / t}$. Therefore, $A_{t} \leq C_{1} / t$ for some constant $C_{1}$. Recall that the oscillation Osc $\psi_{t}:=\max _{M} \psi_{t}-\min _{M} \psi_{t}$ satisfies

$$
\text { Osc } \psi_{t} \leq(m+1) \iota\left(\psi_{t}\right)+C_{2}+2 m A_{t} V / m !,
$$

with $C_{2} \in \mathbb{R}$ depending only on $\theta$. Hence, there exists a constant $C_{3}$ such that

$$
\operatorname{Osc} \psi_{t} \leq(m+1) \iota\left(\psi_{t}\right)+\frac{C_{3}}{t}
$$

Step 2. Differentiating $(5.2 \mathrm{~b})$ with respect to $t$, we obtain the identity $\square_{\omega_{t}} \dot{\psi}_{t}+t \dot{\psi}_{t}+\psi_{t}+C_{t}=0$ for some $C_{t} \in \mathbb{R}$ on $M$. Hence

$$
\frac{d \iota\left(\psi_{t}\right)}{d t}=-\frac{1}{V} \int_{M} \psi_{t}\left(\square_{\omega_{t}} \dot{\psi}_{t}\right) \omega_{t}^{m}=\int_{M}\left(\square_{\omega_{t}} \dot{\psi}_{t}+t \dot{\psi}_{t}\right)\left(\square_{\omega_{t}} \dot{\psi}_{t}\right) \omega_{t}^{m} \geq 0,
$$

where the last inequality follows from the fact that the first eigenvalue of $-\square \omega_{t}$ is greater than $t$. Therefore, $\iota\left(\psi_{t}\right) \leq \iota\left(\psi_{t_{1}}\right)$ for all $t$, and together with Step 1, we have a constant $C_{4}$ such that

$$
t\left(\operatorname{Osc} \psi_{t}\right) \leq C_{4} \quad \text { for all } t .
$$

Step 3. We set $\tilde{f}_{t}:=-t \psi_{t}-\lambda\left(\psi_{t}\right)+f$. Then $(5.2 \mathrm{~b})$ is rewritten as

$$
\left(\theta+\sqrt{-1} \partial \bar{\partial} \psi_{t}\right)^{m}=\exp \left(\tilde{f}_{t}\right) \theta^{m} .
$$

Integrating this over $M$, we obtain $\int_{M} \theta^{m}=\int_{M} \exp \left(\tilde{f}_{t}\right) \theta^{m}$. Now, by the mean value theorem, $\tilde{f}_{t}\left(p_{t}\right)=0$ for some point $p_{t}$ in $M$. Therefore

$$
\left\|\tilde{f}_{t}\right\|_{C^{0}}=\left\|\tilde{f}_{t}-\tilde{f}_{t}\left(p_{t}\right)\right\| \leq t\left(\operatorname{Osc} \psi_{t}\right)+2\|f\|_{C^{0}} \leq C_{4}+2\|f\|_{C^{0}}
$$

In view of $(1)$, a result of Yau ([77]) (see also [17; VII] ) now asserts that Osc $\psi_{t}$ is bounded from above by some constant $C_{5}$. Hence, by setting $\tilde{\psi}_{t}:=\psi_{t}-\psi_{t}\left(p_{t}\right) \in C^{\infty}(M)_{\mathbb{R}}$, we have

$$
\left\|\tilde{\psi}_{t}\right\|_{C^{0}} \leq C_{5} .
$$


Since $\lambda\left(\tilde{\psi}_{t}\right)=\lambda\left(\psi_{t}\right)-\psi_{t}\left(p_{t}\right)$, it now follows that

$$
0=\tilde{f}_{t}\left(p_{t}\right)=-(1+t) \psi_{t}\left(p_{t}\right)-\lambda\left(\tilde{\psi}_{t}\right)+f\left(p_{t}\right)
$$

Therefore

$$
\begin{aligned}
\left|\psi_{t}\left(p_{t}\right)\right|-\|f\|_{C^{0}} & \leq\left|\lambda\left(\tilde{\psi}_{t}\right)\right|=\frac{1}{V}\left|\int_{0}^{1}\left(\int_{M} \tilde{\psi}_{t}\left(\theta+s \sqrt{-1} \partial \bar{\partial} \tilde{\psi}_{t}\right)^{m}\right) d s\right| \\
& \leq \frac{1}{V} \int_{0}^{1}\left(\int_{M} C_{5}\left(\theta+s \sqrt{-1} \partial \bar{\partial} \tilde{\psi}_{t}\right)^{m}\right) d s \leq C_{5} .
\end{aligned}
$$

Together with (2), we can find a constant $C_{6}$ such that $\left\|\psi_{t}\right\|_{C^{0}} \leq C_{6}$ for all $t$, as required.

Q.E.D.

Let $\widetilde{\mathcal{E}}$ be the set of all $\varphi \in \widetilde{\mathcal{K}}$ such that $\Phi(\varphi, 1)=0$. Then by the identification

$$
j: \widetilde{\mathcal{E}} \cong \mathcal{E}, \quad \varphi \leftrightarrow j(\varphi):=\theta+\partial \bar{\partial} \varphi
$$

we can regard each $G^{0}$-orbit $\mathrm{O}$ as a subset $\widetilde{\mathrm{O}}$ of $\widetilde{\mathcal{E}}$. Note that, except the obvious case $H^{0}(M, \mathcal{O}(T M))=\{0\}$, a function $\varphi_{1}$ in $\tilde{\mathcal{E}}$ may fail to extend to a smooth one-parameter family of solutions $\left\{\varphi_{t} ; 1-\varepsilon<\right.$ $t \leq 1\}$ for (5.2a). Because if such a family exists, differentiating (5.2a) with respect to $t$ at $t=1$, we have $\left(\square_{\omega_{1}}+1\right)\left(\dot{\varphi}_{t \mid t=1}\right)=-\varphi_{1}$, where $\omega_{1}:=\theta+\sqrt{-1} \partial \bar{\partial} \varphi_{1}$. Hence $\varphi_{1}$ must satisfy

$$
\int_{M} \varphi_{1} \eta \omega_{1}^{m}=0 \quad \text { for all } \eta \in \operatorname{Ker}_{\mathbb{R}}\left(\square_{\omega_{1}}+1\right) .
$$

We now explain how to find out $\varphi_{1} \in \widetilde{\mathrm{O}}$ satisfying (3). Let $\varphi \in \widetilde{\mathrm{O}}$, and take an arbitrary smooth path $\left\{\sigma_{s} ;-\varepsilon<s<\varepsilon\right\}$ in $\widetilde{\mathrm{O}}$ passing through $\sigma_{0}=\varphi$. Then, through the differentiation of $\Phi\left(\sigma_{s}, 1\right)=0$ with respect to $s$ at $s=1$, it follows that

$$
\dot{\sigma}_{0}\left(:=\dot{\sigma}_{s \mid s=0}\right) \in \operatorname{Ker}_{\mathbb{R}}\left(\square_{j(\varphi)}+1\right)
$$

where by Matsushima's theorem, we have $T_{\varphi}(\widetilde{\mathbf{O}})=\operatorname{Ker}_{\mathbb{R}}\left(\square_{j(\varphi)}+1\right)$. Hence, in view of $\sigma_{0}=\varphi$,

$$
{\frac{d \iota\left(\sigma_{s}\right)}{d s}}_{\mid s=0}=-\frac{1}{V} \int_{M} \varphi\left\{\square_{j(\varphi)}\left(\dot{\sigma}_{0}\right)\right\} j(\varphi)^{m}=\frac{1}{V} \int_{M} \varphi \dot{\sigma}_{0} j(\varphi)^{m} .
$$


Thus, the restriction $\tilde{\iota}:=\iota_{\mid} \tilde{\mathrm{O}}$ of $\iota$ to $\widetilde{\mathrm{O}}$ satisfies

Theorem 5.7. $\varphi \in \widetilde{\mathbf{O}}$ is a critical point for $\tilde{\iota}: \widetilde{\mathbf{O}} \rightarrow \mathbb{R}_{\geq 0}$ if and only if $\int_{M} \varphi \eta j(\varphi)^{m}=0$ for all $\eta \in \operatorname{Ker}_{\mathbb{R}}\left(\square_{j(\varphi)}+1\right)$.

Note that every $\varphi \in \widetilde{\mathrm{O}}$ satisfies the equation $\Phi(\varphi, 1)=0$. Then, as in Step 1 of the proof of Theorem 5.5, upper bounds for $\tilde{\iota}$ give us a priori bounds for $\varphi$ in this equation. Hence, we obtain:

Proposition 5.7.1. $\quad \tilde{\iota}: \widetilde{\mathbf{O}} \rightarrow \mathbb{R}_{\geq 0}$ is a proper map. In particular, there exists a point $\varphi_{1} \in \widetilde{\mathrm{O}}$ at which $\tilde{\imath}$ takes its minimum.

Let $\varphi_{1}$ be as in this proposition. Then $T_{\varphi_{1}}(\widetilde{\mathbf{O}})=\operatorname{Ker}_{\mathbb{R}}\left(\square_{j\left(\varphi_{1}\right)}+1\right)$, and the Hessian

$$
(\operatorname{Hess} \tilde{\imath})_{\varphi_{1}}: \operatorname{Ker}_{\mathbb{R}}\left(\square_{j\left(\varphi_{1}\right)}+1\right) \times \operatorname{Ker}_{\mathbb{R}}\left(\square_{j\left(\varphi_{1}\right)}+1\right) \rightarrow \mathbb{R}
$$

of $\tilde{\iota}$ at $\varphi_{1}$ is clearly positive semidefinite. Moreover, we can realize a critical point for $\tilde{\imath}$ with positive definite Hessian via a small perturbation of our presently fixed $\theta$ (cf. [7]):

Proposition 5.7.2. Taking $\theta \in \mathcal{K}$ sufficiently general, we have a critical point $\varphi_{1} \in \widetilde{\mathrm{O}}$ for $\tilde{\imath}$ such that (Hess $\left.\tilde{\iota}\right)_{\varphi_{1}}$ is positive definite.

We shall now give an outline of the proof of Theorem 5.6. To present our proof in a quite understandable way, we first prove an analogous statement for (5.4), in place of $(5.2 \mathrm{a})$, by making use of only such arguments as applicable to (5.2a). Recall that $\Gamma_{H} \cap\{t=1\}$ is a single parabola $\left\{(x, y) ; y=1-x^{2}\right\}$ sitting in the 2-plane $\{t=1\}$. Clearly, the point $z_{1}:=(0,1)$ on this parabola corresponds to $\varphi_{1}$ on $\widetilde{\mathbf{O}}$ in our original situation in Theorem 5.6. Moreover, the tangent space of the parabola at $(0,1)$ is naturally identified with $\mathbb{R} \oplus\{0\}$, and let

$$
\begin{aligned}
P: \mathbb{R}^{2}(=\mathbb{R} \oplus \mathbb{R}) & \rightarrow \mathbb{R}(=\mathbb{R} \oplus\{0\}), & (x, y) & \mapsto x, \\
1-P: \mathbb{R}^{2}(=\mathbb{R} \oplus \mathbb{R}) & \rightarrow \mathbb{R}(=\{0\} \oplus \mathbb{R}), & (x, y) & \mapsto y,
\end{aligned}
$$

be respectively the projections to the first and the second factors. For $z=(x, y) \in \mathbb{R}$ and $t \in I$ satisfying (5.4), we put

$$
\begin{aligned}
& \hat{z}:=P\left(z-z_{1}\right)=x(=(x, 0)), \\
& \hat{z}:=(1-P)\left(z-z_{1}\right)=y-1(=(0, y-1)),
\end{aligned}
$$

so that $z=z_{1}+\hat{z}+\hat{\hat{z}}$. Hence, (5.4) is rewritten as a combination of the following two equations: 


$$
\begin{aligned}
(1-P) H\left(z_{1}+\hat{z}+\hat{\hat{z}}, t\right) & =0, \\
P H\left(z_{1}+\hat{z}+\hat{\hat{z}}, t\right) & =0 .
\end{aligned}
$$

Since $(1-P) H\left(z_{1}+\hat{z}+\hat{\hat{z}}, t\right)=x^{2}+y-t^{2}=\hat{z}^{2}+\hat{\hat{z}}+1-t^{2}$, these are further expressible as

$$
\begin{aligned}
& \hat{\hat{z}}=\hat{\hat{z}}(\hat{z}, t):=-\hat{z}^{2}+t^{2}-1, \\
& P H\left(z_{1}+\hat{z}+\hat{\hat{z}}(\hat{z}, t), t\right)=0,
\end{aligned}
$$

where in a more general setting, an expression such as (5.8.3) always follows locally from (5.8.1) by the implicit function theorem. Note that $P H\left(z_{1}+\hat{z}+\hat{z}(\hat{z}, t), t\right)=\left(t^{2}-1\right)\left(\hat{z}+t^{2}-t\right)$, which is divisible by $(t-1)$. Hence, (5.8.4) is equivalent to

$$
t=1 \text { or } H_{0}(\hat{z}, t)=0,
$$

where $H_{0}(\hat{z}, t):=P H\left(z_{1}+\hat{z}+\hat{\hat{z}}(\hat{z}, t), t\right) /(t-1)=(t+1)\left(\hat{z}+t^{2}-t\right)$. Now, $H_{0}(\hat{z}, t)=0$ can be solved in the form

$$
\hat{z}=\hat{z}(t)
$$

with $\hat{z}(t):=t-t^{2}$, where in an abstract case, this kind of solution is locally secured by the implicit function theorem in view of the invertibleness of the Jacobian matrix of the mapping $\mathbb{R} \ni \hat{z} \mapsto H_{0}(\hat{z}, 1) \in \mathbb{R}$ at $\hat{z}=0$. Thus, a statement analogous to Theorem 5.6. is true for the equation (5.4).

We now go back to our original equation (5.2a). First, observe that there is nothing to prove if $H^{0}(M, \mathcal{O}(T M))=\{0\}$, since in this case, openness at $t=1$ immediately follows from the invertibleness of $D_{\varphi} \Phi_{\mid\left(\varphi_{1}, 1\right)}=\square_{j\left(\varphi_{1}\right)}+1: C^{2, \alpha}(M)_{\mathbb{R}} \rightarrow C^{0, \alpha}(M)_{\mathbb{B}}$ with $\varphi_{1} \in \tilde{\mathcal{E}}$. Therefore, assume $H^{0}(M, \mathcal{O}(T M)) \neq 0$, and for an arbitrary $G^{0}$-orbit $\mathrm{O}$ in $\mathcal{E}$, let $\varphi_{1} \in \widetilde{\mathrm{O}}$ be as in Proposition 5.7 .2 with $\theta \in \mathcal{K}$ sufficiently general. For simplicity, we put $W:=\operatorname{Ker}_{\mathbb{R}}\left(\square_{j\left(\varphi_{1}\right)}+1\right)$, and let $W_{k}^{\perp}$, $k=0,1,2, \ldots$, be its orthogonal complement in $C^{k, \alpha}(M)_{\mathbb{R}}$ defined by

$$
W_{k}^{\perp}=\left\{\psi \in C^{2, \alpha}(M)_{\mathbb{R}} ; \int_{M} \psi \eta j\left(\varphi_{1}\right)^{m}=0 \text { for all } \eta \in W\right\} \text {. }
$$

This time, we denote by $P$ the natural projection

$$
P: C^{0, \alpha}(M)_{\mathbb{R}}\left(=W \oplus W_{0}^{\perp}\right) \rightarrow W, \quad(\eta \oplus \psi) \mapsto \eta,
$$

to the first factor. For $\varphi \in \widetilde{\mathcal{K}}^{2, \alpha}$, we put 


$$
\begin{aligned}
& \hat{\varphi}:=P\left(\varphi-\varphi_{1}\right) \quad \in W, \\
& \hat{\hat{\varphi}}:=(1-P)\left(\varphi-\varphi_{1}\right) \in W_{2}^{\perp},
\end{aligned}
$$

so that $\varphi=\varphi_{1}+\hat{\varphi}+\hat{\hat{\varphi}}$, and therefore $(5.2 \mathrm{a})$ is rewritten as a combination of the following two equations:

$$
\begin{aligned}
(1-P) \Phi\left(\varphi_{1}+\hat{\varphi}+\hat{\hat{\varphi}}, t\right) & =0, \\
P \Phi\left(\varphi_{1}+\hat{\varphi}+\hat{\hat{\varphi}}, t\right) & =0 .
\end{aligned}
$$

Since the Fréchet derivative $D_{\hat{\hat{\varphi}}} \Pi_{\mid(0,0,1)}$ of $\Pi(\hat{\varphi}, \hat{\hat{\varphi}}, t):=(1-P) \Phi\left(\varphi_{1}+\right.$ $\hat{\varphi}+\hat{\hat{\varphi}}, t)$ with respect to $\hat{\hat{\varphi}}$ at $(\hat{\varphi}, \hat{\hat{\varphi}}, t)=(0,0,1)$ is the invertible operator

$$
W_{2}^{\perp} \ni \psi \longmapsto\left(\square_{j\left(\varphi_{1}\right)}+1\right) \psi \in W_{0}^{\perp},
$$

the implicit function theorem allows us to express (5.8.5), (5.8.6) locally in the form

$$
\begin{aligned}
& \hat{\hat{\varphi}}=\hat{\hat{\varphi}}(\hat{\varphi}, t), \\
& P \Phi\left(\varphi_{1}+\hat{\varphi}+\hat{\hat{\varphi}}(\hat{\varphi}, t), t\right)=0,
\end{aligned}
$$

for some smooth mapping $U \ni(\hat{\varphi}, t) \mapsto \hat{\hat{\varphi}}(\hat{\varphi}, t) \in W_{2}^{\perp}$ with $\hat{\hat{\varphi}}(0,1)=0$ defined in a neighbourhood $U$ of $(\hat{\varphi}, t)=(0,1)$ in $W \times \mathbb{R}$. Note that $T_{\varphi}(\widetilde{\mathbf{O}})=W$. Moreover, $\Phi(\varphi, 1)=0$ for all $\varphi \in \widetilde{\mathbf{O}}$. Hence, it is easily checked that

$$
P \Phi\left(\varphi_{1}+\hat{\varphi}+\hat{\hat{\varphi}}(\hat{\varphi}, t), t\right)=(t-1) \Phi_{0}(\hat{\varphi}, t)
$$

for some smooth map $\Phi_{0}: U \rightarrow W$. It now suffices to show the invertibleness of the Fréchet derivative $D_{\hat{\varphi}} \Phi_{0 \mid q}: W \rightarrow W$ of $\Phi_{0}$ with respect to $\hat{\varphi}$ at $q:=(0,1) \in U$. Recall that, by a direct computation, we have (cf. [7]):

Theorem 5.9. For all $\eta_{1}, \eta_{2} \in W$,

$$
\frac{1}{V} \int_{M} \eta_{1}\left\{D_{\hat{\varphi}} \Phi_{0 \mid q}\left(\eta_{2}\right)\right\} j\left(\varphi_{1}\right)^{m}=(\operatorname{Hess} \tilde{\iota})_{\varphi_{1}}\left(\eta_{1}, \eta_{2}\right)
$$

This together with Proposition 5.7.2 implies $D_{\hat{\varphi}} \Phi_{0 \mid q} \in \mathrm{GL}_{\mathbb{R}}(W)$, as required.

Q.E.D. 


\section{$\S 6$. Existence of Einstein-Kähler metrics I}

In this section, we review the results of Koiso and Sakane $[48,49]$ on the existence of Einstein-Kähler metrics for compactifications of $\mathbb{C}^{*}$ bundles over compact Kähler manifolds. Any compact almost-homogeneous Kähler manifold $Y$ satisfies one of the following:

(1) $Y$ is homogeneous;

(2) the exceptional locus $Y-Y^{0}$ is connected;

(3) $Y-Y^{0}$ is disconnected,

where $Y^{0}$ denotes the open dense orbit in $Y$. Recall that, in (3) above, the open orbit $Y^{0}$ is a $\mathbb{C}^{*}$-bundle over a Kähler $\mathrm{C}$-space, provided that the first Chern class of our compact Kähler manifold is positive (see for instance [1]). Hence, in the case (3), the conditions in Theorem 6.19 below are satisfied, and therefore $Y$ admits an Einstein-Kähler metric if and only if Futaki's obstruction $\mathcal{F}$ for $Y$ vanishes. Therefore, after establishing our main theorem 6.7 , we shall discuss how we can derive Theorem 6.19 from 6.7 (cf. [48; Theorem 4.1]).

Let $p: L \rightarrow M$ be a holomorphic line bundle over a compact Kähler manifold $M$, and $h$ a Hermitian fiber metric on $L$. Denote by $\stackrel{\circ}{L}$ the open subset $L-(0$-section $)$ of $L$ and let $s \in C^{\infty}(\stackrel{\circ}{L})_{\mathbb{R}}$ be the norm of $h$ defined by $s(\ell):=\|\ell\|_{h}(\ell \in \stackrel{\circ}{L})$. Now, consider a function $t=t(s) \in C^{\infty}(\stackrel{\circ}{L})_{\mathbb{R}}$ which depends only on the norm $s$ and is monotone-increasing with respect to $s$. Then the horizontal lift $\tilde{X}$ of a vector field $X$ of $M$ to $\stackrel{L}{L}$ in terms of the canonical Hermitian connection of $L$ is characterized by

$$
p_{*} \tilde{X}=X, \quad \tilde{X} t=(\tilde{J} \tilde{X}) t=0,
$$

where $\tilde{J}$ is the almost complex structure of the total space of $L$. We decompose the group $\mathbb{C}^{*}$ into $S^{1} \times \mathbb{R}^{+}$and define holomorphic vector fields $S, H$ on $\stackrel{\circ}{L}$ generating $S^{1}$-action, $\mathbb{R}^{+}$-action respectively so that

$$
\exp 2 \pi S=\mathrm{id}, \quad H=-\tilde{J} S, \quad H t>0 .
$$

If we denote by $\rho_{L}$ the Ricci form of $L$, then we have

$$
[\tilde{X}, \tilde{Y}]-[\widetilde{X, Y}]=-\rho_{L}(X, Y) S .
$$

Define a Hermitian 2 -form $B$ on $M$ by $\rho_{L}(X, Y)=B(X, J Y)$. It then follows that

$$
[\tilde{X}, \tilde{Y}]-[\widetilde{X, Y}]=-B(X, J Y) S .
$$

Now we consider a Riemannian metric on $\stackrel{\circ}{L}$ of the form

$$
\tilde{g}=d t^{2}+(d t \circ \widetilde{J})^{2}+p^{*} g_{t}
$$


where $\left\{g_{t}\right\}$ is a one-parameter family of Riemannian metrics on $M$. Define a function $u$ on $\stackrel{\circ}{L}$ depending only on $t$ by

$$
u(t)^{2}=\tilde{g}(H, H) .
$$

Lemma 6.1 (see [48]). The metric $\tilde{g}$ on $\stackrel{\circ}{L}$ is a Kähler metric if and only if each $g_{t}$ is a Kähler metric on $M$ satisfying

$$
\frac{d}{d t} g_{t}=-u(t) B
$$

In particular, if we assume that the range of $t$ contains 0 and put

$$
U=\int_{0}^{t} u(t) d t
$$

then

$$
g_{t}=g_{0}-U B
$$

Throughout this section, we assume that $\tilde{g}, B$ and the range of $t$ satisfy the following conditions:

(1) $\tilde{g}$ is a Kähler metric,

(2) the range of $t$ contains 0 and

(3) the eigenvalues of $B$ with respect to $g_{0}$ are constant on $M$.

Note that the condition 3 ) does not depend on the choice of the origin for $t$. Now, let $\left(z^{1}, \cdots, z^{m}\right)$ be a system of holomorphic local coordinates

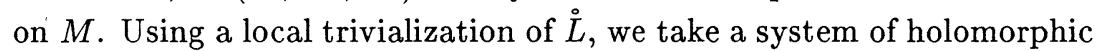
local coordinates $\left(z^{0}, \cdots, z^{m}\right)$ on $\stackrel{\circ}{L}$ such that $\partial / \partial z^{0}=H-\sqrt{-1} S$. Let $\widehat{\partial}_{\alpha}, \widehat{\partial}_{\bar{\alpha}}(0 \leq \alpha \leq m)$ be the partial differentiations $\partial / \partial z^{\alpha}, \partial / \partial \bar{z}^{\alpha}$ on $\stackrel{\circ}{L}$ and $\partial_{\alpha}, \partial_{\bar{\alpha}}(1 \leq \alpha \leq m)$ the partial differentiations $\partial / \partial z^{\alpha}, \partial / \partial \bar{z}^{\alpha}$ on $M$. We now define a new function $q$ on $\stackrel{\circ}{L}$ by

$$
q=\operatorname{det}\left(g_{0}^{-1} \cdot g_{t}\right) \text {. }
$$

Here $g_{0}^{-1} g_{t}$ is regarded as a global section of the endomorphism bundle $\operatorname{End}(T M)$ of $T(M)$.

Then by (6.1.3) and condition (3), $q$ depends only on $t$.

Lemma 6.2. We have

$$
\begin{aligned}
& d t(H)=u, \quad(d t \circ \widetilde{J})\left(\widehat{\partial}_{\beta}\right)=\sqrt{-1} \widehat{\partial}_{\beta} t, \\
& \quad \text { and } \\
& \tilde{g}_{0 \overline{0}}=2 u^{2}, \quad \tilde{g}_{\beta \overline{0}}=2 u \cdot \widehat{\partial}_{\beta} t, \quad \tilde{g}_{\alpha \bar{\beta}}=g_{t \alpha \bar{\beta}}+2 \widehat{\partial}_{\alpha} t \cdot \widehat{\partial}_{\bar{\beta}} t,
\end{aligned}
$$


where $\alpha, \beta=1,2, \cdots, m$. In particular,

$$
\operatorname{det}(\tilde{g})=2 u^{2} \cdot q \cdot \operatorname{det}\left(g_{0}\right)
$$

Now we assume that

$$
\widehat{\partial}_{\alpha} t=\widehat{\partial}_{\bar{\beta}} t=0
$$

on a fiber of $\stackrel{\circ}{L}$ over $M$. Then we have the following:

Lemma 6.3. Let $f$ be a function on $\stackrel{\circ}{L}$ depending only on $t$. Then, at the fiber,

$$
\begin{aligned}
& \widehat{\partial}_{0} \widehat{\partial}_{\overline{0}} f=u \cdot \frac{d}{d t}\left(u \cdot \frac{d f}{d t}\right), \\
& \widehat{\partial}_{\beta} \widehat{\partial}_{\overline{0}} f=0, \\
& \widehat{\partial}_{\alpha} \widehat{\partial}_{\bar{\beta}} f=-\frac{1}{2} u \cdot B_{\alpha \bar{\beta}} \cdot \frac{d f}{d t} .
\end{aligned}
$$

Moreover, the Ricci tensor $\tilde{R}=\operatorname{Ric}(\tilde{g})$ of $\tilde{g}$ at each point of the fibre is given by

$$
\begin{aligned}
& \widetilde{R}_{0 \overline{0}}=-u \cdot \frac{d}{d t}\left(u \cdot \frac{d}{d t} \log \left(u^{2} \cdot q\right)\right), \\
& \widetilde{R}_{\beta \overline{0}}=0 \\
& \widetilde{R}_{\alpha \bar{\beta}}=R_{0 \alpha \bar{\beta}}+\frac{1}{2} u \cdot \frac{d}{d t} \log \left(u^{2} \cdot q\right) \cdot B_{\alpha \bar{\beta}},
\end{aligned}
$$

where $R_{0}=\operatorname{Ric}\left(g_{0}\right)$ denotes the Ricci tensor of $g_{0}$.

Next, we assume that a compactification $\widehat{L}$ of $\stackrel{\circ}{L}$ satisfies the following conditions $A_{1}$ ) and $A_{2}$ ).

Condition $\left.\mathrm{A}_{1}\right)$. Let $(\min t, \max t)$ be the range of $t$. The function $t$ extends to a continuous function on $\hat{L}$ with range $[\min t, \max t]$, and the subsets $M_{\min }, M_{\max }$ of $\widehat{L}$ defined by $t=\min t, t=\max t$ are complex submanifolds of $\widehat{L}$ with codimension $D_{\min }, D_{\max }$ respectively. Moreover, the Kähler metric $\tilde{g}$ extends to a Kähler metric on $\widehat{L}$, which is also denoted by $\tilde{g}$.

Condition $\mathrm{A}_{2}$ ). (1) The Kähler form of the metric $\tilde{g}$ on $\widehat{L}$ represents the first Chern class of $\widehat{L}$. (2) The eigenvalues of $\operatorname{Ric}\left(g_{0}\right)$ with respect to $g_{0}$ are constant on $M$. 
Under Condition $\mathrm{A}_{1}$ ), we see that the function $t-\min t$ gives the distance from $M_{\min }$ in $\widehat{L}$, and that the function $u$ have the Taylor expansion with the first term $t-\min t$, since a fiber $\stackrel{\circ}{L}_{x}$ is compactified to $S_{x}^{2}$. Thus the function $U$ is extended to a $C^{\infty}$-function on a neighbourhood of $M_{\min }$. Therefore, $U$ may be regarded as a $C^{\infty}$-function on $\widehat{L}$, by a similar observation for $M_{\max }$. Moreover we have

Lemma 6.4. A function on $\stackrel{\circ}{L}$ depending only on $t$ extends to a $C^{\infty}$ function on $\widehat{L}$ if and only if it extends smoothly over $\min U$ and $\max U$ as a function in $U$, where $[\min U, \max U]$ denotes the range of $U$ on $\widehat{L}$.

The above (1) in Condition $A_{2}$ ) is equivalent to the existence of a $C^{\infty}$ function $f$ on $\widehat{L}$ such that

$$
\widetilde{R}_{\alpha \bar{\beta}}-\tilde{g}_{\alpha \bar{\beta}}=\widehat{\partial}_{\alpha} \widehat{\partial}_{\bar{\beta}} f \quad(0 \leq \alpha, \beta \leq m) .
$$

Since $\tilde{g}$ is $S^{1}$-invariant, so is $f$, and $f$ may be regarded as a function on $[\min t, \max t] \times M$. Let $f(t)$ be a function on $M$ defined by $f(t)(x)=$ $f(t, x)$. Consider equation (6.5.1) for $\alpha=\beta=0$. Using Lemmas 6.2 and 6.3 , we see that

$$
u \cdot \frac{d}{d t} \log \left(u^{2} \cdot q\right)+2 U+u \cdot \frac{d}{d t} f
$$

is a function on $M$. Now change the variable from $t$ to $U$ and put

$$
\varphi(U)=u^{2}, \quad Q(U)=q .
$$

Note that the function $Q$ is a polynomial in $U$ by (6.1.3) and (6.2.1). Since $u^{2}$ is a $C^{\infty}$ function on $\widehat{L}$, the functions $\varphi$ and $Q$ can be extended smoothly over $\min U$ and $\max U$ by Lemma 6.4 . In view of the identity $d / d t=d U / d t \cdot d / d U$, the function (6.5.2) is given by

$$
\frac{d}{d U} \varphi+\frac{\varphi}{Q} \cdot \frac{d}{d U} Q+2 U+H f
$$

Now, let the variable $U$ tend to $\min U$. Then $H \rightarrow 0$ and thus $H f \rightarrow 0$. We also have $\varphi(U) \rightarrow 0$ and

$$
\frac{d}{d U} \varphi=\frac{d t}{d U} \cdot \frac{d}{d t} \varphi=\frac{1}{u} \cdot 2 u \frac{d u}{d t}=2 \frac{d u}{d t} \rightarrow 2 .
$$

Thus $\varphi(U)$ has the Taylor expansion with the first term $2(U-\min U)$. Since the codimension of $M_{\min }$ in $\widehat{L}$ is $D_{\min }$, the Hermitian metric $g_{t}$ on 
$M$ converges to a Hermitian form on $M$ of rank $m-D_{\min }+1$ when $t$ tends to $\min t$. Hence the function $Q(U)$ has the Taylor expansion with the first term $a(U-\min U)^{D_{\min }^{-1}}(a \neq 0)$, and thus the term $\varphi Q^{-1} \cdot d Q / d U$ has the Taylor expansion with the first term

$$
\frac{2(U-\min U) \cdot\left(D_{\min }-1\right)(U-\min U)^{D_{\min }-2}}{(U-\min U)^{D_{\min }-1}}=2\left(D_{\min }-1\right) .
$$

Therefore the function $(6.5 .4)$ on $M$ is a constant $2+2\left(D_{\min }-1\right)+$ $2 \min U=2\left(\min U+D_{\min }\right)$. By a similar observation at $U=\max U$, this constant coincides with $2\left(\max U-D_{\max }\right)$. Moving the origin of $U$ and $t$ if necessary, we may assume that

$$
\min U=-D_{\min }
$$

Then we have

$$
\max U=D_{\max },
$$

and the function (6.5.4) vanishes identically. In particular, the term $d f / d t$ is a function depending only on $t$ (or $U$ ). By Lemma 6.4 , the equation (6.5.1) for $\alpha=0$ and $\beta>0$ holds automatically. Now we consider the equation (6.5.1) for $\alpha, \beta>0$. Under the assumption (6.3.1), we see that

$$
\begin{aligned}
\widetilde{R}_{\alpha \bar{\beta}}-\tilde{g}_{\alpha \bar{\beta}} & =R_{0 \alpha \bar{\beta}}+\frac{1}{2} u \cdot \frac{d}{d t} \log \left(u^{2} \cdot q\right) \cdot B_{\alpha \bar{\beta}}-g_{0 \alpha \bar{\beta}}+U \cdot B_{\alpha \bar{\beta}}, \\
\widehat{\partial}_{\alpha} \widehat{\partial}_{\bar{\beta}} f & =\widehat{\partial}_{\alpha}\left(\widehat{\partial}_{\bar{\beta}} t \cdot \frac{d}{d t} f+\partial_{\bar{\beta}} f\right)=\widehat{\partial}_{\alpha} \widehat{\partial}_{\bar{\beta}} t \cdot \frac{d}{d t} f+\partial_{\alpha} \partial_{\bar{\beta}} f \\
& =-\frac{1}{2} u \cdot B_{\alpha \bar{\beta}} \cdot \frac{d f}{d t}+\partial_{\alpha} \partial_{\bar{\beta}} f .
\end{aligned}
$$

Therefore we have

$$
\partial_{\alpha} \partial_{\bar{\beta}} f=R_{0 \alpha \bar{\beta}}-g_{0 \alpha \bar{\beta}},
$$

since (6.5.2) vanishes. By (2) in Condition $\mathrm{A}_{2}$ ), the signature of the right-hand side is constant on $M$ and thus $f$ is constant on $M$, that is, it depends only on $t$. In particular, we see that $\operatorname{Ric}\left(g_{0}\right)=g_{0}$. Summing up our result, we obtain

Lemma 6.5. Under Conditions $\mathrm{A}_{1}$ ) and $\mathrm{A}_{2}$ ), we also assume that $\min U=-D_{\min }$. Then $f$ is a function depending on $t$ alone, and it satisfies

$$
\frac{d}{d U} \varphi+\frac{\varphi}{Q} \cdot \frac{d}{d U} Q+2 U+H f=0
$$


Moreover we have $\max U=D_{\max }$ and $\operatorname{Ric}\left(g_{0}\right)=g_{0}$. In particular, the Kähler metric $\tilde{g}$ on $\widehat{L}$ is an Einstein metric if and only if we have the identities $\operatorname{Ric}\left(g_{0}\right)=g_{0}$ and

$$
\frac{d}{d U} \varphi+2 U+\frac{\varphi}{Q} \cdot \frac{d}{d U} Q=0
$$

Now we consider Futaki's obstruction to the existence of EinsteinKähler metrics ([25], see also §2). First, observe that

$$
\begin{aligned}
\int_{\widehat{L}} H f \omega_{\tilde{g}}^{m+1} & =\int_{\min t}^{\max t} H f \cdot \operatorname{Vol}\left(M, g_{t}\right) u d t \\
& =\operatorname{Vol}\left(M, g_{0}\right) \int_{\min t}^{\max t} H f \cdot u q d t \\
& =\operatorname{Vol}\left(M, g_{0}\right) \int_{\min U}^{\max U} H f \cdot Q d U \\
& =-\operatorname{Vol}\left(M, g_{0}\right) \int_{\min U}^{\max U}\left(\frac{d}{d U} \varphi+U+\frac{\varphi}{Q} \cdot \frac{d}{d U} Q\right) Q d U \\
& =-\operatorname{Vol}\left(M, g_{0}\right)\left\{[Q \varphi]_{\min U}^{\max U}+2 \int_{\min U}^{\max U} Q U d U\right\} \\
& =-2 \operatorname{Vol}\left(M, g_{0}\right) \int_{-D_{\min }}^{D_{\max }} Q U d U .
\end{aligned}
$$

In our case, by setting

$$
\mathcal{F}_{0}(\widehat{L})=\int_{-D_{\min }}^{D_{\max }} Q U d U
$$

we can express Futaki's obstruction in the following form:

Lemma 6.6. Under Conditions $\mathrm{A}_{1}$ ) and $\mathrm{A}_{2}$ ), if $\widehat{L}$ admits an Einstein-Kähler metric, then $\mathcal{F}_{0}(\widehat{L})=0$.

Recall that one of our main purposes in this section is to show the converse of Lemma 6.6.

Theorem 6.7 ([48]). Let $M$ be a compact Einstein-Kähler manifold with Kähler form in the de Rham cohomology class $2 \pi c_{1}(M)_{\mathbb{R}}$ and let $L$ be a Hermitian line bundle over $M$. Assume that the eigenvalues of the Ricci form $B$ for $L$ are constant on $M$ with respect to the metric 
$g_{0}$ and that, on a compactification $\widehat{L}$ of $\stackrel{\circ}{L}$, there is a Kähler metric $\tilde{g}$ of the form (6.1.1) with Kähler class $2 \pi c_{1}(\widehat{L})_{\mathbb{R}}$. Then the following three conditions are equivalent.

(1) $\widehat{L}$ admits an Einstein-Kähler metric.

(2) Futaki's obstruction is zero.

(3) The integral $\mathcal{F}_{0}(\widehat{L})$ is zero.

Outline of Proof. It is enough to show that if the integral $\mathcal{F}_{0}(\widehat{L})$ is zero then $\widehat{L}$ admits an Einstein-Kähler metric. Let $\phi$ be a function on $\widehat{L}$ depending only on $t$ and we seek a condition that the metric $\tilde{h}$ on $\widehat{L}$ defined by

$$
\tilde{h}_{\alpha \bar{\beta}}=\tilde{g}_{\alpha \bar{\beta}}+\widehat{\partial}_{\alpha} \widehat{\partial}_{\bar{\beta}} \phi
$$

is an Einstein-Kähler metric. We also assume that the metric $\tilde{h}$ satisfies the condition (6.1.1) and there is a parameter $s$ (depending only on $t$ ) such that $\tilde{h}$ has the form

$$
\widetilde{h}=d s^{2}+(d s \circ \tilde{J})^{2}+p^{*} h_{s}
$$

on $\stackrel{\circ}{L}$. We take parameters $v, V, \psi$ for $\widetilde{h}$ which correspond to $u, U, \varphi$ for $\tilde{g}$. We construct a solution such that $h_{0}=g_{0}, \min V=-D_{\min }$ and $\max V=D_{\max }$. By Lemma 6.5 , the condition that the metric $\tilde{h}$ is Einstein-Kähler reduces to the equation

$$
\frac{d}{d V} \phi+2 V+\frac{\phi}{Q} \cdot \frac{d}{d V} Q=0
$$

and it has a solution given by

$$
\phi(V)=-2 \int_{-D_{\min }}^{V} V Q(V) d V / Q(V) .
$$

Then the function $\phi(V)$ is positive on $\left(-D_{\min }, D_{\max }\right)$, extends smoothly over $-D_{\min }$ and has the Taylor expansion with first term $2\left(V+D_{\min }\right)$. Since the integral $\mathcal{F}_{0}(\widehat{L})$ is zero, we have

$$
\phi(V)=2 \int_{V}^{D_{\max }} V Q(V) d V / Q(V)
$$

and so $\phi(V)$ extends smoothly over $D_{\max }$ and has the Taylor expansion with first term $-2\left(V-D_{\max }\right)$. Now $H$ coincides with $u \cdot d / d t$ and $v \cdot d / d s$. 
Thus we have

$$
\frac{d s}{d t}=\frac{v}{u}
$$

and

$$
\begin{gathered}
\frac{d V}{d U}=\frac{\phi(V)}{\varphi(U)} \\
\int \frac{d V}{\phi(V)}=\int \frac{d U}{\varphi(U)}
\end{gathered}
$$

Therefore, using the Taylor expansion at $-D_{\min }$, we see that

$$
\begin{aligned}
& \log \left(V+D_{\min }\right)+C^{\infty} \text { function of } V \\
= & \log \left(U+D_{\min }\right)+C^{\infty} \text { function of } U,
\end{aligned}
$$

hence $V$ extends smoothly over $-D_{\min }$ as a function of $U$. In the same way, $V$ extends smoothly over $D_{\max }$ as a function of $U$, and thus $V$ extends to a $C^{\infty}$ function in $U$ defined on an open set containing $\left[-D_{\min }, D_{\max }\right]$. To see that $\widetilde{h}$ is a metric on $\widehat{L}$, we first see that $\phi$ is a $C^{\infty}$ function on $\widehat{L}$ by using (6.7.1), Lemmas 6.2 and 6.3 . Thus $\widetilde{h}$ is a $C^{\infty}$ symmetric 2 -form on $\widehat{L}$. On $\stackrel{\circ}{L}$, it is easy to see that $\widetilde{h}$ is positive definite, since $Q(V)>0$. Take tubular neighbourhoods of $M_{\min }$ and $M_{\max }$ in $\widehat{L}$. By comparing $\tilde{g}$ and $\tilde{h}$ on the tubular neighbourhoods, we see that $\tilde{h}$ is positive definite (see [48; Theorem 4.1] for details). Q.E.D.

We shall now discuss a couple of examples of $\widehat{L}$ which satisfy Conditions $\mathrm{A}_{1}$ ) and $\mathrm{A}_{2}$ ) above.

Let $p: L \rightarrow M$ be a holomorphic line bundle over a compact Kähler manifold $M$ with a Hermitian metric $h$. We put

$$
u(t)=a \cos \frac{t-t_{0}}{a} \quad \text { with } t-t_{0} \in\left(-\frac{\pi a}{2}, \frac{\pi a}{2}\right) \text { for } a>0,
$$

and define the function $U(t)$ by (6.1.2). Then we have

$$
U(t)=a^{2} \sin \frac{t-t_{0}}{a}+b
$$

and $t_{0}$ is given by

$$
a^{2} \sin \frac{t_{0}}{a}=b
$$

Take a Kähler metric $g_{0}$ on $M$ and assume that each $g_{t}$ defined by (6.1.3) is positive definite. As before, we consider a Kähler metric $\tilde{g}$ on $\stackrel{\circ}{L}$ of the form (6.1.1) satisfying $u(t)^{2}=\tilde{g}(H, H)$. 
Lemma 6.8. Let $s$ be the norm of the Hermitian line bundle $p$ : $L \rightarrow M$. Then on $\stackrel{\circ}{L}$, we have

$$
U(t)=\frac{\left(a^{2}+b\right) s^{2}+\left(b-a^{2}\right)}{s^{2}+1}
$$

by replacing $t(s)$ by $t(c s)$ for some positive constant $c$ if necessary.

As our examples, we first consider the case of $\mathrm{P}^{1}$-bundles. Let us assume that $M$ admits an Einstein-Kähler metric $g_{0}$ with $\operatorname{Ric}\left(g_{0}\right)=g_{0}$ and that the holomorphic line bundle $L$ has a Hermitian metric such that the eigenvalues of the Hermitian 2-form $B$ with respect to $g_{0}$ are all constant on $M$ having the absolute values less than 1 . Put $\widehat{L}=$ $\mathbb{P}(1 \oplus L)=\mathbb{P}\left(L^{-1} \oplus 1\right)$. Note that $L$ and $L^{-1}$ are canonically imbedded as complex submanifolds of $\widehat{L}$ so that $L \cap L^{-1}=\stackrel{\circ}{L}$.

Theorem 6.9 ([48]). Under the assumptions as above, we have $c_{1}(\widehat{L})>0$ and

$$
\mathcal{F}_{0}(\widehat{L})=\int_{-1}^{1} U \operatorname{det}\left(\mathrm{id}-U g_{0}^{-1} B\right) d U
$$

Moreover $\widehat{L}$ admits an Einstein-Kähler metric if and only if $\mathcal{F}_{0}(\widehat{L})=0$.

Proof. Put $u(t)=\cos t\left(t \in\left(-\frac{\pi}{2}, \frac{\pi}{2}\right)\right)$ and $U(t)=\sin t$. Then each $g_{t}=g_{0}-U(t) B$ is positive definite by our assumption. Consider a Kähler metric $\tilde{g}$ on $\stackrel{\circ}{L}$ of the form (6.1.1) satisfying $u(t)^{2}=\tilde{g}(H, H)$. By Lemma 6.8, the function $U$ defined on the open subset $\stackrel{\circ}{L}$ of $\widehat{L}$ is extended to a $C^{\infty}$ function on $\widehat{L}$, which is also denoted by $U$, such that the range of $U$ on the complex submanifold $L$ is $[-1,1)$ and the range of $U$ on the complex submanifold $L^{-1}$ is $(-1,1]$. Now we claim that there are $C^{\infty}$ functions $f_{0}: L \rightarrow \mathbb{R}, f_{\infty}: L^{-1} \rightarrow \mathbb{R}$ and Kähler forms $\underline{\omega}_{1}, \underline{\omega}_{2}$ on $M$ such that the Kähler form $\omega_{\tilde{g}}$ on $\stackrel{\circ}{L}$ can be written as follows:

$$
\omega_{\tilde{g}}=p^{*} \underline{\omega}_{1}-2 \sqrt{-1} \partial \bar{\partial} f_{0}=p^{*} \underline{\omega}_{2}-2 \sqrt{-1} \partial \bar{\partial} f_{\infty} .
$$

Any $C^{\infty}$ function $f_{0}: L \rightarrow \mathbb{R}$ as in (6.9.1) must satisfy

$$
2 u^{2}=-2 u \frac{d}{d t}\left(u \frac{d f_{0}}{d t}\right)
$$


by Lemmas 6.2 and 6.3 . Since $\varphi(U)=u^{2}$ and $d / d t=u \cdot d / d U$, the equation (6.9.2) can be written as

$$
1=-\frac{d}{d U}\left(\varphi(U) \frac{d f_{0}}{d U}\right) .
$$

Thus for some constant $C \in \mathbb{R}$,

$$
\frac{d f_{0}}{d U}=-\frac{(U+C)}{\varphi(U)} .
$$

Since $\varphi(U)=u^{2}=\cos ^{2} t=1-\sin ^{2} t=1-U^{2}$, we have

$$
\frac{d f_{0}}{d U}=\frac{-1}{1-U}
$$

by setting $\mathrm{C}=1$, and thus $f_{0}=\log (1-U)$. Since the range of $U$ on the complex submanifold $L$ is $[-1,1), f_{0}=\log (1-U)$ is a $C^{\infty}$ function on $L$. By Lemma 6.3, we have

$$
\widehat{\partial}_{\alpha} \widehat{\partial}_{\bar{\beta}} f_{0}=-\frac{1}{2} u \cdot B_{\alpha \bar{\beta}} \cdot \frac{d f_{0}}{d t}=-\frac{1}{2} B_{\alpha \bar{\beta}} \cdot \varphi(U) \frac{d f_{0}}{d U} .
$$

Since $\varphi(U) \cdot d f_{0} / d U=-(U+1)$, we have

$$
g_{0}-U B-\varphi(U) \frac{d f_{0}}{d U} B=g_{0}+B
$$

which is a Kähler metric on $M$ by our assumption. Put $\underline{\omega}_{1}=\omega_{g_{0}+B}$. Then

$$
\omega_{\tilde{g}}=p^{*} \underline{\omega}_{1}-2 \sqrt{-1} \partial \bar{\partial} f_{0} .
$$

In the same way, we have

$$
\omega_{\tilde{g}}=p^{*} \underline{\omega}_{2}-2 \sqrt{-1} \partial \bar{\partial} f_{\infty}
$$

where $\underline{\omega}_{2}=\omega_{g_{0}-B}$ and $f_{\infty}=\log (1+U)$. Since $f_{0}=\log (1-U)=$ $-\log \left(s^{2}+1\right)+\log 2$,

$$
p^{*} \underline{\omega}_{1}-2 \sqrt{-1} \partial \bar{\partial} f_{0}=p^{*} \underline{\omega}_{1}+2 \sqrt{-1} \partial \bar{\partial} \log \left(s^{2}+1\right) .
$$

Now the right-hand side of (6.15) is a Kähler form on a neighborhood of 0 -section of $p: L \rightarrow M$. Therefore we can extend $\tilde{g}$ on $\stackrel{\circ}{L}$ to a Kähler metric on $L$, and also to a Kähler metric on $L^{-1}$ similarly. Hence the metric $\tilde{g}$ satisfies Condition $A_{1}$ ). Now we claim that $\tilde{g}$ also satisfies (1) in 
Condition $\left.\mathrm{A}_{2}\right)$. More precisely, there is a $C^{\infty}$ function $F(U)$ on $[-1,1]$ such that

$$
\operatorname{Ric}\left(\omega_{\tilde{g}}\right)-\omega_{\tilde{g}}=2 \sqrt{-1} \partial \bar{\partial} F
$$

By the proof of Lemma 6.5, (6.9.4) is equivalent to the equation

$$
\varphi \frac{d}{d U} \log (\varphi Q)+2 U+\varphi \frac{d F}{d U}=0 .
$$

By solving this equation, we have

$$
F=-\log (\varphi Q)+\int \frac{-2 U}{\varphi} d U
$$

Since $\varphi(U)=1-U^{2}$, we have $F=-\log Q$. Since $Q(U)>0$ on $[-1,1]$, $F$ is a $C^{\infty}$ function on $[-1,1]$ and hence $F$ is a $C^{\infty}$ function on $\widehat{L}$. Therefore $c_{1}(\widehat{L})>0$.

Example 6.10 (cf. [71]). Let $n_{1}$ and $n_{2}$ be positive integers and $L_{i}$ be the tautological line bundle $\mathcal{O}(-1)$ over the complex projective space $\mathbb{P}^{n_{i}}$ of dimension $n_{i}$ for $i=1,2$. Denote by $p_{i}: \mathbb{P}^{n_{1}} \times \mathbb{P}^{n_{2}} \rightarrow \mathbb{P}^{n_{i}}$ the projection to the $i$-th factor. For given positive integers $a$ and $b$, let $L$ be the holomorphic line bundle on $\mathbb{P}^{n_{1}} \times \mathbb{P}^{n_{2}}$ given by

$$
L=p_{1}^{*} L_{1}^{-a} \otimes p_{2}^{*} L_{2}^{b}
$$

Put $\widehat{L}=\mathbb{P}(1 \oplus L)$. If $a \leq n_{1}$ and $b \leq n_{2}$, then $L$ satisfies the assumptions of Theorem 6.9 , and hence $c_{1}(\widehat{L})>0$ and

$$
\mathcal{F}_{0}(\widehat{L})=\int_{-1}^{1} U\left(1+\frac{a}{n_{1}+1} U\right)^{n_{1}}\left(1-\frac{b}{n_{2}+1} U\right)^{n_{2}} d U
$$

In particular, if $n_{1}=n_{2}$ and $a=b$, then $\mathcal{F}_{0}(\widehat{L})=0$ (cf. Proposition 2.8) and hence $\widehat{L}$ admits an Einstein-Kähler metric.

Example 6.11 (cf. [48]). Let $N$ be a compact Einstein-Kähler manifold with a Kähler metric $g_{0}$ such that $\operatorname{Ric}\left(g_{0}\right)=g_{0}$ and let $L_{N}$ be a holomorphic line bundle on $N$ such that $c_{1}\left(L_{N}\right)=a c_{1}(N)$ with $0<a<1$. Put $M=N \times N$ and let $p_{i}: M \rightarrow N$ be the $i$-th projection for $i=1,2$. Let $L$ be a holomorphic line bundle on $M$ given by

$$
L=p_{1}{ }^{*} L_{N}{ }^{-1} \otimes p_{2}{ }^{*} L_{N}
$$


Then $\widehat{L}=\mathbb{P}(1 \oplus L)$ admits an Einstein-Kähler metric, since

$$
\mathcal{F}_{0}(\widehat{L})=\int_{-1}^{1} U(1-a U)^{n}(1+a U)^{n} d U=0
$$

where $n=\operatorname{dim}_{\mathbb{C}} N$.

6.12. Now we discuss examples of $\widehat{L}$ which are not $\mathbb{P}^{1}$-bundles. Let $E$ be a holomorphic vector bundle of rank $r$ over a compact complex manifold $N$. The complex projective bundle $\mathbb{P}(E)$ associated to $E$ is defined as follows. Let $\mathbb{C}^{*}$ act freely on $E-(0$-section $)$ by scalar multiplication. Then $\mathbb{P}(E)$ is the quotient complex manifold

$$
E-(0-\text { section }) / \mathbb{C}^{*} \text {. }
$$

Thus a point of $\mathbb{P}(E)$ over $x \in N$ represents a complex line in the fiber $E_{x}$ of $E$ at $x$. We organize various spaces and maps by the following commutative diagram:

$$
\mathbb{P}(E) \underset{\varphi \searrow}{\stackrel{p}{\longleftarrow} E-(0 \text {-section })} \underset{N}{\hookrightarrow} E
$$

Via the projection $\varphi: \mathbb{P}(E) \rightarrow N$, we pull back $E$ to the induced vector bundle $\varphi^{*} E$ over $\mathbb{P}(E)$ of rank $r$. We define the tautological line bundle $L(E)$ over $\mathbb{P}(E)$ as a subbundle of $\varphi^{*} E$ as follows. The fiber $L(E)_{\xi}$ at $\xi \in \mathbb{P}(E)$ is the complex line in $E_{\varphi(\xi)}$ represented by $\xi$. Note also that, if $L$ is a holomorphic line bundle over $N$, we have $L(E \otimes L)=L(E) \otimes \varphi^{*} L$ as holomorphic line bundles over $\mathbb{P}(E \otimes L)=\mathbb{P}(E)$.

Our compact complex manifold $Y$ can be obtained from the $\mathbb{P}^{1}$ bundle $\mathbb{P}(1 \oplus L)$ by blowing down 0 -section $M_{1}$ of $L$ to a compact complex manifold $N_{1}$ and $\infty$-section $M_{2}$ of $L$ to a compact complex manifold $N_{2}$. More precisely, our manifold $Y$ is constructed as follows. Let $N_{1}, N_{2}$ be compact complex manifolds and consider holomorphic vector bundles $E_{1}$ of rank $\ell+1 \geqslant 2$ over $N_{1}, E_{2}$ of rank $k+1 \geqslant 2$ over $N_{2}$. We also assume that the total spaces $\mathbb{P}\left(E_{1}\right)$ and $\mathbb{P}\left(E_{2}\right)$ of projective bundles coincide as complex manifolds, which is denoted by $M$, and there are holomorphic line bundles $L_{1}^{\prime}$ over $N_{1}$ and $L_{2}^{\prime}$ over $N_{2}$ such that the tautological line bundles $L\left(E_{1} \otimes L_{1}^{\prime-1}\right)$ over $\mathbb{P}\left(E_{1} \otimes L_{1}^{\prime-1}\right)$ and $L\left(E_{2} \otimes L_{2}^{\prime-1}\right)$ over $\mathbb{P}\left(E_{2} \otimes\right.$ $\left.L_{2}^{\prime-1}\right)$ satisfy $L\left(E_{1} \otimes L_{1}^{\prime-1}\right)^{-1}=L\left(E_{2} \otimes L_{2}^{\prime}{ }^{-1}\right)$. More precisely, there is a holomorphic bundle isomorphism 


$$
L\left(E_{1} \otimes L_{1}^{\prime-1}\right)^{-1} \rightarrow L\left(E_{2} \otimes L_{2}^{\prime-1}\right)
$$

compatible with the identification:

$$
\mathbb{P}\left(E_{1} \otimes L_{1}^{\prime-1}\right) \cong \mathbb{P}\left(E_{1}\right) \cong \mathbb{P}\left(E_{2}\right) \cong \mathbb{P}\left(E_{2} \otimes L_{2}^{\prime-1}\right) .
$$

We also consider the $P^{1}$-bundle

$$
X:=\mathbb{P}\left(1 \oplus L\left(E_{1} \otimes L_{1}^{\prime-1}\right)\right)=\mathbb{P}\left(L\left(E_{2} \otimes L_{2}^{\prime-1}\right) \oplus 1\right)
$$

over $M=\mathbb{P}\left(E_{1} \otimes L_{1}^{\prime-1}\right)=\mathbb{P}\left(E_{2} \otimes L_{2}^{\prime-1}\right)$. Note that complex submanifolds $M_{1}, M_{2}$ of $X$, defined by the 0 -section of $L\left(E_{1} \otimes L_{1}^{\prime-1}\right)$ and 0 -section of $L\left(E_{2} \otimes L_{2}^{\prime-1}\right)$, are identified with $M=\mathbb{P}\left(E_{1} \otimes L_{1}^{\prime-1}\right)$ and $M=\mathbb{P}\left(E_{2} \otimes L_{2}^{\prime-1}\right)$ respectively. Now, we organize various spaces and maps by the following diagram:

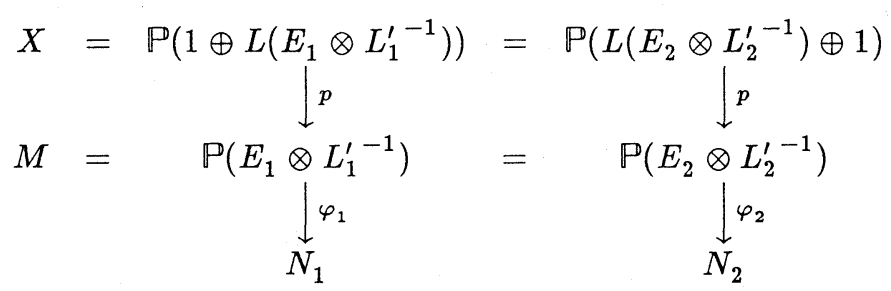

Then the following lemma is a special case of results of Nakano [68], Fujiki and Nakano [24].

Lemma 6.14. There exist a compact manifold $Y$ containing $N_{1}$, $N_{2}$ as complex submanifolds and a holomorphic map $\Phi: X \rightarrow Y$ in such a way that $(X, \Phi)$ is a monoidal transform of $Y$ with centers $N_{1}, N_{2}$ and that $M_{1}=\mathbb{P}\left(E_{1} \otimes L_{1}^{\prime-1}\right)=\Phi^{-1}\left(N_{1}\right), M_{2}=\mathbb{P}\left(E_{2} \otimes L_{2}^{\prime-1}\right)=\Phi^{-1}\left(N_{2}\right)$ are the exceptional sets in $X$.

Remark 6.15. (1) Note that, over a projective bundle $\mathbb{P}(E)$, the total space of the tautological line bundle $L(E)$ is obtained from $E$ by blowing up the 0 -section of $E$ in $\mathbb{P}(E)$. Note also that $\mathbb{P}\left(1 \oplus L\left(E_{1} \otimes\right.\right.$ $\left.\left.L_{1}^{\prime-1}\right)\right)$ is obtained by gluing open complex submanifolds $L\left(E_{1} \otimes L_{1}^{\prime-1}\right)$ and $L\left(E_{2} \otimes L_{2}^{\prime-1}\right)$ via the identification

$$
\begin{gathered}
L\left(E_{1} \otimes{L_{1}^{\prime}}^{-1}\right) \cap L\left(E_{2} \otimes L_{2}^{\prime-1}\right) \\
=L\left(E_{1} \otimes L_{1}^{\prime-1}\right)-(0 \text {-section })=L\left(E_{2} \otimes L_{2}^{\prime-1}\right)-(0 \text {-section }) .
\end{gathered}
$$


Thus $Y$ is a union of the canonically imbedded open submanifolds $E_{1} \otimes$ $L_{1}^{\prime-1}$ and $E_{2} \otimes L_{2}^{\prime-1}$ with the intersection

$$
\begin{gathered}
E_{1} \otimes L_{1}^{\prime-1} \cap E_{2} \otimes L_{2}^{\prime-1} \\
=E_{1} \otimes L_{1}^{\prime-1}-(0 \text {-section })=E_{2} \otimes L_{2}^{\prime-1}-(0 \text {-section }),
\end{gathered}
$$

which is also identified with

$$
L\left(E_{1} \otimes L_{1}^{\prime-1}\right)-(0 \text {-section })=L\left(E_{2} \otimes L_{2}^{\prime-1}\right)-(0 \text {-section }) .
$$

(2) In general, a compact Kähler manifold is not necessarily blown down to a Kähler manifold (cf: $[65]$ ). Therefore, we have to show that our $Y$ is a Kähler manifold. Denote by $\stackrel{\circ}{E}$ the open subset $E-(0$-section) of $E$. Then, in view of the identification $\stackrel{\circ}{E}=\stackrel{\circ}{L}(E):=L(E)-(0$-section), any Hermitian metric $h_{1}$ on $E$ induces naturally a Hermitian metric $h$ on $L(E)$ by

$$
h_{x}(v, w)=\left(h_{1}\right)_{\varphi(x)}(v, w), \quad v, w \in \stackrel{\circ}{L}(E)_{x}=\stackrel{\circ}{E}_{\varphi(x)},
$$

for every $x \in \mathbb{P}(E)$. Note here that, in general, fiber metrics on $L(E)$ do not necessarily define Hermitian metrics on $E$, whereas there is a natural one-to-one correspondence between complex Finsler structures in $E$ and Hermitian structures in $L(E)$ (see [45]).

Now, by setting $a^{2}=(\ell+k+2) / 2$ and $b=(k-\ell) / 2$ in Lemma 6.8, we obtain the following:

Lemma 6.16. Taking $M$ to be a Kähler manifold, let $N_{1}, N_{2}$, $E_{1}, E_{2}, L_{1}^{\prime}, L_{2}^{\prime}$ and $Y$ be as in (6.12), (6.13) and (6.14). We assume that there are Hermitian metrics $h_{1}$ on $E_{1} \otimes L_{1}^{\prime-1}$ and $h_{2}$ on $E_{2} \otimes L_{2}^{\prime-1}$ such that, as functions on $\stackrel{\circ}{L}\left(E_{1} \otimes L_{1}^{\prime}{ }^{-1}\right)=\stackrel{\circ}{L}\left(E_{2} \otimes L_{2}^{\prime-1}\right)$, the square of the Hermitian norm on $L\left(E_{2} \otimes L_{2}^{\prime}{ }^{-1}\right)$ induced by $h_{2}$ depends only on the square of the Hermitian norm on $L=L\left(E_{1} \otimes L_{1}^{\prime-1}\right)$ induced by $h_{1}$. Assume further that we can construct a Kähler metric $\tilde{g}$ on $\stackrel{\circ}{L}$ as in Lemma 6.1. Moreover, choose the function $t$ in such a way that the end points of the corresponding range $(-(\ell+1), k+1)$ of $U$ is given by $\ell+1=\operatorname{rank} E_{1}=\operatorname{codim}_{Y} N_{1}$ and $k+1=\operatorname{rank} E_{2}=\operatorname{codim}_{Y} N_{2}$, where codimensions are taken over $\mathbb{C}$. Then the function $U$ on the open subset $\stackrel{\circ}{L}$ of the compact complex manifold $Y$ extends to a $C^{\infty}$ function (denoted also by $U$ ) on $Y$ such that the range of $U$ on the complex submanifold 
$E_{1} \otimes L_{1}^{\prime-1}$ is $[-(\ell+1), k+1)$ and the range of $U$ on $E_{2} \otimes L_{2}^{\prime-1}$ is $(-(\ell+1), k+1]$.

We now seek the condition for the mertric $\tilde{g}$ on $\stackrel{\circ}{L}=\stackrel{\circ}{L}\left(E_{1} \otimes L_{1}^{\prime-1}\right)=$ $\stackrel{\circ}{L}\left(E_{2} \otimes L_{2}^{\prime-1}\right)$ to be of the form

$$
\omega_{\tilde{g}}=\left(\varphi_{1} \circ p\right)^{*} \underline{\omega}_{1}-2 \sqrt{-1} \partial \bar{\partial} f_{0}=\left(\varphi_{2} \circ p\right)^{*} \underline{\omega}_{2}-2 \sqrt{-1} \partial \bar{\partial} f_{\infty}
$$

for some Kähler forms $\underline{\omega}_{i}$ on $N_{i}, i=1,2$, and some $f_{0}, f_{\infty} \in C^{\infty}(\stackrel{\circ}{L})_{\mathbb{R}}$, where we require both $f_{0}$ and $f_{\infty}$ to be able to depend on $t$ alone. As to this, we can prove the following Lemmas by the same arguments as in the proof of Theorem 6.9.

Lemma 6.17. Under the same assumption as in Lemma 6.16, suppose that the Kähler metric $g_{0}$ on $M=\mathbb{P}\left(E_{1} \otimes L_{1}^{\prime-1}\right)=\mathbb{P}\left(E_{2} \otimes L_{2}^{\prime-1}\right)$ and the Hermitian form $B$ on $M$ satisfy the identities $g_{0}+(\ell+1) B=$ $\varphi_{1}{ }^{*} \underline{g}_{1}$ and $g_{0}-(k+1) B=\varphi_{2}{ }^{*} \underline{g}_{2}$ for some Kähler metrics $\underline{g}_{1}$ on $N_{1}$ and $\underline{g}_{2}$ on $N_{2}$. Let $\underline{\omega}_{i}, i=1,2$, be the Kähler form on $N_{i}$ associated with $\underline{g}_{i}$. Then there are $C^{\infty}$ function $f_{0}: E_{1} \otimes L_{1}^{\prime-1} \rightarrow \mathbb{R}$ and $f_{\infty}: E_{2} \otimes L_{2}^{\prime-1} \rightarrow \mathbb{R}$, both depending on $t$ alone, such that on $\stackrel{\circ}{L}$,

$$
\omega_{\tilde{g}}=\left(\varphi_{1} \circ p\right)^{*} \underline{\omega}_{1}-2 \sqrt{-1} \partial \bar{\partial} f_{0}=\left(\varphi_{2} \circ p\right)^{*} \underline{\omega}_{2}-2 \sqrt{-1} \partial \bar{\partial} f_{\infty} .
$$

Lemma 6.18. Under the same assumption as in Lemmas 6.16 and 6.17 , the Kähler metric $\tilde{g}$ on $\stackrel{\circ}{L}$ can be extended to a Kähler metric on the complex manifold $Y$, and therefore $Y$ satisfies Condition $\mathrm{A}_{1}$ ).

We assume further that the eigenvalues of $B$ with respect to $g_{0}$ are constant on $M$, where we always regard $B$ as a Hermitian form for the holomorphic tangent bundle $T(M)$ of $M$. Note that the identities in the assumption for Lemma 6.17 implies that $-(\ell+1)^{-1},(k+1)^{-1}$ are both (global) eigenvalues of $B$ with respect to $g_{0}$ with multiplicity $\ell$, $k$, because $\varphi_{1}{ }^{*} \underline{g}_{1}, \varphi_{2}{ }^{*} \underline{g}_{2}$ are positive semi-definite Hermitian forms of nullity $\ell, k$ respectively. Thus the function $Q(U)=q=\operatorname{det}\left(g_{0}^{-1} g_{t}\right)$ on $\stackrel{\circ}{L}$ is expressible as

$$
Q(U)=\operatorname{det}\left(1-U g_{0}^{-1} B\right)=\left(1+\frac{U}{\ell+1}\right)^{\ell}\left(1-\frac{U}{k+1}\right)^{k} Q_{1}(U)
$$

for some polynomial $Q_{1}=Q_{1}(U)$ in $U$ which is nowhere vanishing on $[-(\ell+1), k+1]$. We finally obtain 
Theorem 6.19 (cf. [49]). Under the same assumptions as in Lemmas 6.16 and 6.17 , suppose that the eigenvalues of $B$ with respect to $g_{0}$ are constant on $M$. Assume further that the Ricci tensor $\operatorname{Ric}\left(\dot{g}_{0}\right)$ for the Kähler metric $g_{0}$ on $M$ is equal to $g_{0}$. Then the first chern class $c_{1}(Y)$ of $Y$ is positive and the Ricci form $\operatorname{Ric}\left(\omega_{\tilde{g}}\right)$ for the Kähler metric $\tilde{g}$ on $Y$ satisfies

$$
\operatorname{Ric}\left(\omega_{\tilde{g}}\right)-\omega_{\tilde{g}}=2 \sqrt{-1} \partial \bar{\partial} F
$$

for some $C^{\infty}$ function $F=F(U)$ on $[-(\ell+1), k+1]$. Therefore $\tilde{g}$ satisfies (1) in Condition $\mathrm{A}_{2}$ ). Moreover $Y$ admits an Einstein-Kähler metric if and only if

$$
\int_{-(\ell+1)}^{k+1} U Q(U) d U=0
$$

Several examples satisfying the assumptions in Theorem 6.19 will be given in Appendix.

\section{$\S 7 . \quad$ Existence of Einstein-Kähler metrics II}

The purpose of this section is to give a brief survey of recent results of Siu [73], Tian and Yau [74, 75] on the existence of Einstein-Kähler metrics for del Pezzo surfaces and a special type of Fermat hypersurfaces.

Let $M$ be a compact complex connected manifold with $c_{1}(M)>0$ and, as in $\S 5$, let $\mathcal{K}$ be the set of all Kähler forms in the de Rham cohomology class $2 \pi c_{1}(M)_{\mathbb{R}}$. For each $\omega \in \mathcal{K}$, we denote by $\gamma_{\omega}$ the supremum of $\lambda$ 's in $\mathbb{R}$ such that $\operatorname{Ric}(\omega)-\lambda \omega$ is positive definite everywhere on $M$. Then

$$
\gamma_{M}:=\sup \left\{\gamma_{\omega} ; \omega \in \mathcal{K}\right\}
$$

is a biholomorphic invariant of $M$. Moreover, let $\mathcal{E}_{M}$ be the moduli space

$$
\{\omega \in \mathcal{K} ; \operatorname{Ric}(\omega)=\omega\}
$$

of Einstein-Kähler forms on $M$. Then from the definition of $\gamma_{M}$, we easily see the following:

(1) $0<\gamma_{M} \leq 1$

(2) If $\mathcal{E}_{M} \neq \phi$, then $\gamma_{M}=1$.

Note that, as remarked by Siu $[73 ;(4.7)]$, there actually exists an $M$ such that $\gamma_{M} \neq 1$. Let $\mathcal{M}_{n}$ be the set of all compact complex connected $n$-dimensional manifolds (modulo biholomorphisms) with positive first Chern class. For instance, complex surfaces in $\mathcal{M}_{2}$ are called del Pezzo 
surfaces. Now, we put $\gamma(n):=\inf \gamma_{M}$ and $C(n):=\sup c_{1}(M)^{n}[M]$, where the infimum and supremum are taken over all $M$ 's in $\mathcal{M}_{n}$. It then follows that:

Theorem 7.1 (cf. Tian and Yau [75]). $C(n)<+\infty$ if and only if $\gamma(n)>0$.

Proof. In view of Myers's theorem [67] and Bishop's inequality [11], "if" part follows easily from the fact that the uniform lower bound $\gamma(n)>0$ of $\operatorname{Ric}(\omega)$ for $\omega \in \mathcal{K}$ yields a uniform upper bound of the total volume $\int_{M} \omega^{n} / n !\left(=\left\{2 \pi c_{1}(M)\right\}^{n}[M] / n !\right)$. Conversely, we assume $C(n)<+\infty$. Then Matsusaka's big theorem (cf. Matsusaka [62], Kollár and Matsusaka [51]) allows us to choose positive integers $r$ and $s$, depending only on $C(n)$ and $n$, such that we have a projective embedding $i: M \hookrightarrow \mathbb{P}^{s}(\mathbb{C})$ with $i^{*} \mathcal{O}_{\mathbb{P}^{s}}(1)=K_{M}^{\otimes-r}$. Let $\omega \in \mathcal{K}$ be the Kähler form on $M$ obtained from $\mathbb{P}^{s}(\mathbb{C})$ by pulling back a constant multiple of the Fubini-Study form. Then via the estimate of appropriate Lelong numbers (cf. [75; Theorem 5.3]),

$$
\gamma_{M} \geq \gamma_{\omega} \geq\left(r^{n-1} c_{1}(M)^{n}[M]\right)^{-1} \geq\left(r^{n-1} C(n)\right)^{-1},
$$

whenever $M$ is in $\mathcal{M}_{n}$. Thus, $\gamma(n) \geq\left(r^{n-1} C(n)\right)^{-1}>0$.

Q.E.D.

Let $V^{\prime}, V^{\prime \prime}, M^{\prime}, M^{\prime \prime}$ be compact complex connected manifolds. Then $V^{\prime}$ is called a complex analytic deformation of $V^{\prime \prime}$ if there exists a proper smooth morphism between complex connected manifolds such that $V^{\prime}, V^{\prime \prime}$ are its fibres. Moreover, $M^{\prime}$ and $M^{\prime \prime}$ are said to be of the same deformation type if there exist a finite number of complex connected manifolds $V_{0}, V_{1}, \ldots, V_{m}$ such that

(1) $V_{0}=M^{\prime}$ and $V_{m}=M^{\prime \prime}$;

(2) $V_{i}$ is a complex analytic deformation of $V_{i-1}$ for $1 \leq i \leq m$.

We now recall the following conjecture in algebraic geometry:

Conjecture 7.2. For each $n$, there are only a finite number of deformation types in $\mathcal{M}_{n}$.

This conjecture is true for $n \leq 3$. For instance, the case $n=3$ follows immediately from the classification of Fano threefolds by Iskovskih $[39,40]$, Mori and Mukai [66]. For $n \geq 4$, however, this is still open. Note that, by Matsusaka's big theorem (cf. [62], [51]), $\mathcal{M}_{n}$ consists of finite deformation types if and only if $C(n)<+\infty$. Hence, in view of Theorem 7.1, the above conjecture is true if we can show the following: 
Conjecture 7.3 (cf. Siu [73], Tian and Yau [75]). The inequality $\gamma(n)>0$ holds for all $n$.

Let us go back to our original study of Einstein-Kähler metrics. For $d \in \mathbb{Z}$ with $1 \leq d \leq 9$, let $\mathcal{M}_{2, d}$ be the set of all compact complex connected surfaces $M$ (modulo biholomorphisms) satisfying both $c_{1}(M)>0$ and $c_{1}(M)^{2}[M]=d$. For any positive integer $r$, let $\mathcal{S}_{r}$ be the set of all complex surfaces (modulo biholomorphisms) obtained from $\mathbb{P}^{2}(\mathbb{C})$ by blowing up $r$ points no three of which are colinear and no six of which lie on a conic. Note that, for $1 \leq r \leq 4$, the set $\mathcal{S}_{r}$ consists of a single element. Now by the classification of compact complex surfaces,

$$
\mathcal{M}_{2}=\bigcup_{d=1}^{9} \mathcal{M}_{2, d}
$$

where

$$
\begin{aligned}
& \mathcal{M}_{2,9}=\left\{\mathbb{P}^{2}(\mathbb{C})\right\}, \\
& \mathcal{M}_{2,8}=\left\{\mathbb{P}^{1}(\mathbb{C}) \times \mathbb{P}^{1}(\mathbb{C})\right\} \cup \mathcal{S}_{1}, \\
& \mathcal{M}_{2, d}=\mathcal{S}_{9-d}, \quad 1 \leq d \leq 7 .
\end{aligned}
$$

Clearly, both $\mathbb{P}^{2}(\mathbb{C})$ and $\mathbb{P}^{1}(\mathbb{C}) \times \mathbb{P}^{1}(\mathbb{C})$ carry Einstein-Kähler metrics, while for $M$ in $S_{1} \cup S_{2}$, we have $\mathcal{E}_{M}=\phi$ by the non-vanishing of the corresponding Futaki's obstruction. Recently, Siu [73], Tian and Yau $[74,75]$ established the following existence results of Einstein-Kähler metrics:

Theorem 7.4. (1) (cf. Siu [73], Tian and Yau [74, 75]). If $M \in$ $\mathcal{S}_{3} \cup \mathcal{S}_{4}$, then $\mathcal{E}_{M} \neq \phi$.

(2) (cf. [73], [74, 75]). For $0<m \in \mathbb{Z}$, if $M$ is a Fermat hypersurface $\left\{\left(z_{0}: z_{1}: \cdots: z_{m+1}\right) \in \mathbb{P}^{m+1}(\mathbb{C}) ; \sum_{i=0}^{m+1} z_{i}^{d}=0\right\}$ of degree $d=m$ or $m+1$, then $\mathcal{E}_{M} \neq \phi$.

(3) (cf. Tian and Yau [75]). If $M \in \mathcal{S}_{5} \cup \mathcal{S}_{8}$, then $\mathcal{E}_{M} \neq \phi$.

(4) (cf. [75]). When $r \in\{6,7\}$, we have $\mathcal{E}_{M} \neq \phi$ for some $M \in \mathcal{S}_{r}$.

The proof of this theorem, for instance, by Tian and Yau $[74,75]$ essentially depends on the estimate of appropriate Lelong numbers, though we do not give it here. Note that (3) above is not explicitly stated in [75]. However, one can easily deduce (3) from [75] as follows: If $M \in S_{5}$, then $M$ is written as a nonsingular complete intersection

$$
M=\left\{\left(z_{0}: z_{1}: \cdots: z_{4}\right) \in \mathbb{P}^{4}(\mathbb{C}) ; \Sigma_{i=0}^{4} z_{i}^{2}=\Sigma_{i=0}^{4} a_{i} z_{i}^{2}=0\right\},
$$


where $a_{i} \in \mathbb{C}, 0 \leq i \leq 4$, are such that $a_{i} \neq a_{j}$ whenever $i \neq j$. Hence, by [75; Theorem 2.3], we have $\mathcal{E}_{M} \neq \phi$. Moreover, if $M \in \mathcal{S}_{8}$, then $M$ always admits Bertini's involution $1 \neq \sigma \in \operatorname{Aut}(M)$ (cf. Demazure [21; p. 68], Koitabashi [50]). Therefore, by [75; Theorem 4.2], we again have $\mathcal{E}_{M} \neq \phi$. It is now quite plausible that the following conjecture is true:

Conjecture 7.5. Whenever $M \in \mathcal{S}_{6} \cup \mathcal{S}_{7}$, we have $\mathcal{E}_{M} \neq \phi$.

If this conjecture is affirmative, then in view of Theorem 7.4, one can easily show that a del Pezzo surface can carry an Einstein-Kähler metric only when the corresponding Futaki's obstruction vanishes. At present, however, this conjecture is neither disproved nor given an affirmative answer.*

\section{Appendix}

In this appendix, we give examples of $Y$ satisfying the assumptions of Theorem 6.19 (cf. Example 12). Examples of $\widehat{L}$ (cf. Example 8) that account for Theorem 6.9 will also be given.

We first recall known facts on compact simply connected homogeneous Kähler manifolds, called Kähler C-spaces. Let $\Pi$ be a Dynkin diagram and $\Pi_{0}$ a subdiagram of $\Pi$. The pair $\left(\Pi, \Pi_{0}\right)$ is said to be effective if $\Pi_{0}$ does not contain any irreducible component of $\Pi$. Let $\Sigma$ be the root system with the fundamental root system $\Pi$. Choose a lexicographic order $>$ on $\Sigma$ such that the set of simple roots with respect to $>$ coincides with $\Pi$. Take a compact semi-simple Lie algebra $\mathfrak{g}_{u}$ with the root system $\Sigma$ and let $\mathfrak{t}$ be a maximal abelian subalgebra of $\mathfrak{g}_{u}$. Denote by $\mathfrak{g}$ and $\mathfrak{h}$ the complexification of $\mathfrak{g}_{u}$ and $\mathfrak{t}$ respectively. We identify a weight of $\mathfrak{g}$ relative to the Cartan subalgebra $\mathfrak{h}$ with an element of $\sqrt{-1} \mathfrak{t}$ by the duality defined by the Killing form $($,$) of \mathfrak{g}$. In particular, the root system $\Sigma$ of $\mathfrak{g}$ relative to $\mathfrak{h}$ is a subset of $\sqrt{-1} \mathfrak{t}$. Let $\left\{\Lambda_{\alpha}\right\}_{\alpha \in \Pi} \subset \sqrt{-1} \mathrm{t}$ be the fundamental weights of $\mathfrak{g}$ corresponding to $\Pi$ :

$$
\frac{2\left(\Lambda_{\alpha}, \beta\right)}{(\beta, \beta)}=\delta_{\alpha \beta} \text {. }
$$

Let $\Sigma^{+}$be the set of all positive roots and $\left\{\Pi_{0}\right\}_{\mathbb{Z}}$ the subgroup of $\sqrt{-1} \mathrm{t}$ generated by $\Pi_{0}$. Put $\Sigma_{0}=\Sigma \cap\left\{\Pi_{0}\right\}_{\mathbb{Z}}$. We define a subalgebra $\mathfrak{u}$ of $\mathfrak{g}$ by

$$
\mathfrak{u}=\mathfrak{h}+\sum_{\alpha \in \Sigma_{0} \cup \Sigma^{+}} \mathfrak{g}_{\alpha}
$$

* Note added in proof: We were recently informed that Tian solved Conjecture 7.5 affirmatively. 
where $\mathfrak{g}_{\alpha}$ is the root space of $\mathfrak{g}$ for $\alpha \in \Sigma$. Let $G$ be a simply connected complex Lie group whose Lie algebra is $\mathfrak{g}$, and let $U$ be the connected (closed) complex Lie subgroup of $G$ generated by u. Put $M=G / U$. Then it is known that the complex manifold $M=G / U$ is compact, simply connected and admits a homogeneous Kähler metric. Let $G_{u}$ be the compact connected subgroup of $G$ generated by $\mathfrak{g}_{u}$. Put $K=G_{u} \cap U$. Then $K$ is connected, $G_{u}$ acts on $M$ transitively and $M=G / U=G_{u} / K$ as a smooth manifold. This homogeneous complex manifold $M$ is said to be associated to the pair $\left(\Pi, \Pi_{0}\right)$ of Dynkin diagrams.

We define a subspace $c$ of $\sqrt{-1} t$ by

$$
\mathfrak{c}=\sum_{\alpha \in \Pi-\Pi_{0}} \mathbb{R} \Lambda_{\alpha}
$$

Then $\sqrt{-1} \mathfrak{c}$ coincides with the center of the Lie algebra $\mathfrak{k}$ of $K$. We also define lattices $Z$ of $\sqrt{-1} \mathfrak{t}$ and $Z_{\mathfrak{c}}$ of $\mathfrak{c}$ by

$$
Z=\left\{\lambda \in \sqrt{-1} \mathrm{t} \mid \frac{2(\lambda, \alpha)}{(\alpha, \alpha)} \text { is an integer for each } \alpha \in \Sigma\right\}
$$

and

$$
Z_{\mathrm{c}}=Z \cap \mathrm{c} .
$$

Let $\mathfrak{m}$ be the orthogonal complement of $\mathfrak{k}$ in $\mathfrak{g}_{u}$ with respect to the Killing form $(,) ; \mathfrak{g}_{u}=\mathfrak{k}+\mathfrak{m}$. The subspace $\mathfrak{m}$ is $K$-invariant under the adjoint action and identified with the tangent space $T_{o}(M)$ of $M$ at the origin $o \in M$. Put

$$
\Sigma_{\mathrm{m}}^{+}=\Sigma^{+}-\Sigma_{0}, \quad \Sigma_{\mathrm{m}}^{-}=-\Sigma_{\mathrm{m}}^{+} .
$$

We define $K$-invariant subspace $\mathfrak{m}^{ \pm}$of $\mathfrak{g}$ by

$$
\mathfrak{m}^{ \pm}=\sum_{\alpha \in \Sigma_{m}^{ \pm}} \mathfrak{g}_{-\alpha} .
$$

Then the complexification $\mathfrak{m}^{\mathbb{C}}$ of $\mathfrak{m}$ is the direct sum:

$$
\mathfrak{m}^{\mathbb{C}}=\mathfrak{m}^{+}+\mathfrak{m}^{-} \text {. }
$$

We denote by $X \rightarrow \bar{X}$ the complex conjugation of $\mathfrak{g}$ with respect to the real form $\mathfrak{g}_{u}$. Then $\mathfrak{m}^{\mp}=\overline{\mathfrak{m}^{ \pm}}$. We choose $E_{\alpha} \in \mathfrak{g}_{\alpha}$ for $\alpha \in \Sigma$ with the following properties and fix them from now on:

$$
\left[E_{\alpha}, E_{-\alpha}\right]=-\alpha, \quad\left(E_{\alpha}, E_{-\alpha}\right)=-1, \quad \overline{E_{\alpha}}=E_{-\alpha} \quad \text { for } \alpha \in \Sigma \text {. }
$$


Let $\left\{\omega^{\alpha}\right\}_{\alpha \in \Sigma}$ be the linear forms on $\mathfrak{g}$ dual to $\left\{E_{\alpha}\right\}_{\alpha \in \Sigma}$, that is, linear forms defined by

$$
\begin{cases}\omega^{\alpha}(\mathfrak{h}) & =\{0\} \\ \omega^{\alpha}\left(E_{\beta}\right) & =\delta_{\alpha \beta}\end{cases}
$$

We define a cone $\mathfrak{c}^{+}$in $\mathfrak{c}$ by

$$
\mathfrak{c}^{+}=\left\{\lambda \in \mathfrak{c} \mid(\lambda, \alpha)>0 \text { for each } \alpha \in \Pi-\Pi_{0}\right\}
$$

and put $Z_{\mathrm{c}}^{+}=Z \cap \mathfrak{c}^{+}$. Then we have

$$
\mathfrak{c}^{+}=\sum_{\alpha \in \Pi-\Pi_{0}} \mathbb{R}^{+} \Lambda_{\alpha}, \quad Z_{\mathrm{c}}^{+}=\sum_{\alpha \in \Pi-\Pi_{0}} \mathbb{Z}^{+} \Lambda_{\alpha} .
$$

Moreover, the cone $\mathfrak{c}^{+}$is characterized by

$$
\mathfrak{c}^{+}=\left\{\lambda \in \mathfrak{c} \mid(\lambda, \alpha)>0 \text { for each } \alpha \in \Sigma_{\mathrm{m}}^{+}\right\} .
$$

We define elements $\delta_{\mathrm{m}}, \delta$ of $\sqrt{-1} \mathrm{t}$ by

$$
\delta_{\mathrm{m}}=\frac{1}{2} \sum_{\alpha \in \Sigma_{\mathrm{m}}^{+}} \alpha, \quad \delta=\frac{1}{2} \sum_{\alpha \in \Sigma^{+}} \alpha
$$

respectively. It is known that $2 \delta_{\mathrm{m}} \in Z_{\mathrm{c}}^{+}$and $\delta=\sum_{\alpha \in \Pi} \Lambda_{\alpha}$. Now we recall the following facts.

Fact 1. Let $M=G / U=G_{u} / K$ be the compact homogeneous complex manifold associated to an effective pair $\left(\Pi, \Pi_{0}\right)$ of Dynkin diagrams. Then we have the following:

1) For $\lambda \in \mathfrak{c}$,

$$
g(\lambda)=\frac{1}{2 \pi} \sum_{\alpha \in \Sigma_{\mathrm{m}}^{+}}(\lambda, \alpha) \omega^{-\alpha} \cdot \overline{\omega^{-\alpha}}
$$

defines a $G_{u}$-invariant real covariant symmetric tensor field of degree 2 on $M$, and the correspondence $\lambda \rightarrow g(\lambda)$ gives a bijection from $\mathrm{c}^{+}$to the set of $G_{u}$-invariant Kähler metrics on $M$.

2) For the Kähler metric $g$ corresponding to $\lambda \in \mathfrak{c}^{+}$, the Kähler form $\omega$, the Ricci tensor $\operatorname{Ric}(g)$ and the Ricci form $\operatorname{Ric}(\omega)$ are given by

$$
\omega=\frac{\sqrt{-1}}{2 \pi} \sum_{\alpha \in \Sigma_{\mathrm{m}}^{+}}(\lambda, \alpha) \omega^{-\alpha} \wedge \overline{\omega^{-\alpha}},
$$




$$
\operatorname{Ric}(g)=2 \sum_{\alpha \in \Sigma_{\mathrm{m}}^{+}}\left(2 \delta_{\mathrm{m}}, \alpha\right) \omega^{-\alpha} \cdot \overline{\omega^{-\alpha}}
$$

$$
\operatorname{Ric}(\omega)=2 \sqrt{-1} \sum_{\alpha \in \Sigma_{\mathrm{m}}^{+}}\left(2 \delta_{\mathrm{m}}, \alpha\right) \omega^{-\alpha} \wedge \overline{\omega^{-\alpha}}
$$

Fact 2. For each $\Lambda \in Z_{\mathfrak{c}}$, there is unique holomorphic character $\chi_{\Lambda}$ of $U$ such that

$$
\chi_{\Lambda}(\exp H)=\exp (\Lambda, H) \quad \text { for each } H \in \mathfrak{h} .
$$

Let $L_{\Lambda}$ denote the holomorphic line bundle on $M$ associated to the principal bundle $U \rightarrow G \rightarrow M$ by the character $\chi_{\Lambda}$. The correspondence $\Lambda \rightarrow L_{\Lambda}$ induces an isomorphism from $Z_{\mathrm{c}}$ onto the group $H^{1}\left(M, \mathcal{O}^{*}\right)$ of all holomorphic line bundles on $M$. Moreover, under this isomorphism the subset $-Z_{\mathrm{c}}^{+}$corresponds to the set of all very ample holomorphic line bundles on $M$. The first Chern class $c_{1}\left(L_{\Lambda}\right)$ of $L_{\Lambda}$ contains unique $G_{u}$-invariant 2 -form

$$
-\frac{\sqrt{-1}}{2 \pi} \sum_{\alpha \in \Sigma_{\mathrm{m}}^{+}}(\Lambda, \alpha) \omega^{-\alpha} \wedge \overline{\omega^{-\alpha}}
$$

on $M$.

Let $\Pi$ be a Dynkin diagram and $\Pi_{0}$ a subdiagram of $\Pi$ such that $\Pi_{0}$ is of type $A_{\ell-1}\left(\Pi_{0}=\emptyset\right.$ if $\left.\ell=1\right)$. Consider also a subdiagram $\Pi_{1}$ of $\Pi$ such that $\Pi_{1}$ contains $\Pi_{0}$ as a subdiagram and $\Pi_{1}$ is of type $A_{\ell}$. Put $\Sigma_{1}=\Sigma \cap\left\{\Pi_{1}\right\}_{\mathbb{Z}}$. We define a Lie subalgebra $\mathfrak{p}$ of $\mathfrak{g}$ by

$$
\mathfrak{p}=\mathfrak{h}+\sum_{\alpha \in \Sigma_{1} \cup \Sigma^{+}} \mathfrak{g}_{\alpha}
$$

We denote by $G / U, G / P$ the Kähler C-spaces associated to the pairs $\left(\Pi, \Pi_{0}\right),\left(\Pi, \Pi_{1}\right)$ of Dynkin diagrams respectively. Put $\left\{\alpha_{0}\right\}=\Pi_{1}-\Pi_{0}$ and $\Lambda_{0}=\Lambda_{\alpha_{0}} \in Z$. We define a subalgebra $\mathfrak{g}(1)$ of $\mathfrak{p}$ by

$$
\mathfrak{g}(1)=\mathfrak{h}+\sum_{\alpha \in \Sigma_{1}} \mathfrak{g}_{\alpha}
$$

and let $G(1)$ be the complex subgroup of $G$ generated by $\mathfrak{g}(1)$. Then there is an irreducible representation $\rho_{\Lambda_{0}}: G(1) \rightarrow \operatorname{GL}\left(V_{\Lambda_{0}}\right)$ of $G(1)$ with the highest weight $\Lambda_{0}$. The representation $\rho_{\Lambda_{0}}$ can be uniquely 
extended to an irreducible representation of $P$, which is also denoted by $\rho_{\Lambda_{0}}: P \rightarrow \operatorname{GL}\left(V_{\Lambda_{0}}\right)$. Note that $\operatorname{dim}_{\mathbb{C}} V_{\Lambda_{0}}=\ell+1$. We denote by $E_{\Lambda_{0}}$ the homogeneous vector bundle over $G / P$ defined by the representation $\rho_{\Lambda_{0}}: P \rightarrow \mathrm{GL}\left(V_{\Lambda_{0}}\right)$ and by $\mathbb{P}\left(E_{\Lambda_{0}}\right)$ the complex projective bundle over $G / P$ associated to the vector bundle $E_{\Lambda_{0}}$. Then $G$ acts on $E_{\Lambda_{0}}$ and $\mathbb{P}\left(E_{\Lambda_{0}}\right)$ in natural ways and we have $\mathbb{P}\left(E_{\Lambda_{0}}\right)=G / U$. Note that $E_{\Lambda_{0}}-$ (0-section) is a $\mathbb{C}^{*}$-bundle over $\mathbb{P}\left(E_{\Lambda_{0}}\right)$. Let $L\left(E_{\Lambda_{0}}\right)$ be the tautological line bundle over $\mathbb{P}\left(E_{\Lambda_{0}}\right)$ associated to the vector bundle $E_{\Lambda_{0}}$ over $G / P$. Then we have an identification:

$$
E_{\Lambda_{0}}-(0 \text {-section })=L\left(E_{\Lambda_{0}}\right)-(0 \text {-section })
$$

Lemma 3. The tautological line bundle $L\left(E_{\Lambda_{0}}\right)$ is the holomorphic line bundle $L_{\Lambda_{0}}$ over $\mathbb{P}\left(E_{\Lambda_{0}}\right)=G / U$ associated to the principal bundle $U \rightarrow G \rightarrow G / U$ by the character $\chi_{\Lambda_{0}}$ of $U$.

Now we recall the following formula for the canonical line bundle of a projective bundle. Let $\tau: E \rightarrow N$ be a holomorphic vector bundle of rank $r$ over a complex manifold $N$ and let $K_{\mathbb{P}(E)}, K_{N}$ denote the canonical line bundle on $\mathbb{P}(E), N$ respectively. Then

$$
K_{\mathbb{P}(E)}=\tau^{*}\left(K_{N} \otimes \operatorname{det} E^{*}\right) \otimes L(E)^{r}
$$

where $\operatorname{det} E^{*}$ denotes the holomorphic line bundle $\wedge^{r} E^{*}$. Applying this formula to compute the first Chern class of $\mathbb{P}\left(E_{\Lambda_{0}}\right)=G / U$, we have

Lemma 4. The element $-2 \delta_{\mathrm{m}} \in-Z_{\mathrm{c}}^{+}$corresponding to the first Chern class $c_{1}\left(\mathbb{P}\left(E_{\Lambda_{0}}\right)\right)$ of $\mathbb{P}\left(E_{\Lambda_{0}}\right)=G / U$ is given by

$$
-2 \delta_{\mathrm{m}}=-(\ell+1) \Lambda_{0}+\sum_{\alpha \in \Pi-\Pi_{1}}-n_{\alpha} \Lambda_{\alpha} \quad \text { for some } n_{\alpha} \in \mathbb{N}
$$

We now consider the triples $\left(\Pi, \Pi_{1}^{1}, \Pi_{0}\right),\left(\Pi, \Pi_{1}^{2}, \Pi_{0}\right)$ of Dynkin diagrams which are one of the following:

(a) The Dynkin diagram $\Pi$ is connected, $\Pi_{0}$ is a subdiagram of $\Pi$ and of type $A_{\ell-1}$, and subdiagrams $\Pi_{1}^{1}, \Pi_{1}^{2}$ of $\Pi$ are of type $A_{\ell}$ containing $\Pi_{0}$ as a subdiagram.

(b) The Dynkin diagram $\Pi$ has two connected components $\Pi(1)$ and $\Pi(2)$, and $\Pi_{0}$ is a subdiagram of $\Pi$ which has also two connected components $\Pi_{0}(1)$ of type $A_{\ell-1}$ and $\Pi_{0}(2)$ of type $A_{k-1}$. Subdiagram 
$\Pi_{1}^{1}$ (resp. $\Pi_{1}^{2}$ ) of $\Pi$ have also two connected components $\Pi_{1}^{1}(1)$ and $\Pi_{1}^{1}(2)$ (resp. $\Pi_{1}^{2}(1)$ and $\Pi_{1}^{2}(2)$ ) satisfying the following conditions:

(b-1) $\Pi_{1}^{1}(1)$ is a subdiagram of $\Pi(1)$, of type $A_{\ell}$ and contains $\Pi_{0}(1)$ as a subdiagram, and $\Pi_{1}^{1}(2)$ coincides with $\Pi_{0}(2)$.

(b-2) $\Pi_{1}^{2}(2)$ is a subdiagram of $\Pi(2)$, of type $A_{k}$ and contains $\Pi_{0}(2)$ as a subdiagram, and $\Pi_{1}^{2}(2)$ coincides with $\Pi_{0}(1)$.

Example 5. The vertices contained in $\Pi_{0}, \Pi_{1}^{i}-\Pi_{0}, \Pi-\Pi_{1}^{i}$ of a Dynkin diagram $\Pi$ are denoted by $\circ, \bullet, \times$ for $i=1,2$ respectively.
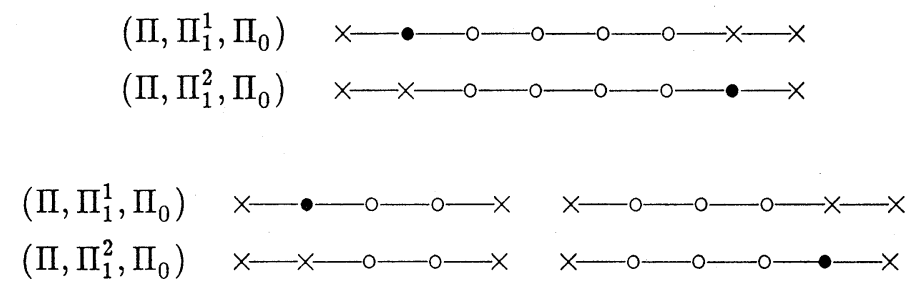

Put $\left\{\alpha_{0}(i)\right\}=\Pi_{1}^{i}-\Pi_{0}$ and $\Lambda_{0}(i)=\Lambda_{\alpha_{0}(i)}$ for $i=1,2$. We consider Kähler C-spaces associated to pairs of Dynkin diagrams and $\mathbb{P}^{1}$-bundles over Kähler C-spaces.

Case (a) We denote by $G / U, G / P_{1}, G / P_{2}$ the Kähler C-spaces associated to the pairs $\left(\Pi, \Pi_{0}\right),\left(\Pi, \Pi_{1}^{1}\right),\left(\Pi, \Pi_{1}^{2}\right)$ respectively, and by $E_{1}$, $E_{2}$ the homogeneous vector bundles $E_{\Lambda_{0}(1)}, E_{\Lambda_{0}(2)}$ over $G / P_{1}, G / P_{2}$ respectively. We have

$$
M=\mathbb{P}\left(E_{1}\right)=\mathbb{P}\left(E_{2}\right)=G / U,
$$

and $L\left(E_{1}\right)=L_{\Lambda_{0}(1)}, L\left(E_{2}\right)=L_{\Lambda_{0}(2)}$ by Lemma 3 . Put $L_{1}=L_{\Lambda_{0}(1)}$ and $L_{2}=L_{\Lambda_{0}(2)}$. Let $\varphi_{1}: M=G / U \rightarrow N_{1}=G / P_{1}, \varphi_{2}: M=G / U \rightarrow$ $N_{2}=G / P_{2}$ be the natural projections. Note that there are holomorphic line bundles $L_{1}^{\prime}$ over $N_{1}=G / P_{1}$ and $L_{2}^{\prime}$ over $N_{2}=G / P_{2}$ such that $\varphi_{1}{ }^{*} L_{1}^{\prime}=L_{2}, \varphi_{2}{ }^{*} L_{2}^{\prime}=L_{1}$. We thus have

$$
L\left(E_{1} \otimes L_{1}^{\prime-1}\right)=L_{1} \otimes L_{2}^{-1}=L\left(E_{2} \otimes L_{2}^{\prime-1}\right)^{-1} .
$$

Note also that the $\mathbb{P}^{1}$-bundle $X$ is given by $\mathbb{P}\left(1 \oplus L_{1} \otimes L_{2}{ }^{-1}\right)$.

Case (b) We denote by $G_{1} / U_{1}, G_{1} / P_{1}, G_{2} / U_{2}, G_{2} / P_{2}$ the Kähler Cspaces associated to the pairs $\left(\Pi(1), \Pi_{0}(1)\right),\left(\Pi(1), \Pi_{1}^{1}(1)\right),\left(\Pi(2), \Pi_{0}(2)\right)$, 
(ח(2), $\left.\Pi_{1}^{2}(2)\right)$ respectively and by $E_{1}, E_{2}$ the homogeneous vector bundles $E_{\Lambda_{0}(1)}, E_{\Lambda_{0}(2)}$ over $G_{1} / P_{1}, G_{2} / P_{2}$ respectively. We regard the vector bundles $E_{1}$ over $G_{1} / P_{1}$ and $E_{2}$ over $G_{2} / P_{2}$ as vector bundles over $N_{1}=G_{1} / P_{1} \times G_{2} / U_{2}$ and $N_{2}=G_{1} / U_{1} \times G_{2} / P_{2}$ respectively, which are also denoted by $E_{1}$ and $E_{2}$ respectively. We have

$$
M=\mathbb{P}\left(E_{1}\right)=\mathbb{P}\left(E_{2}\right)=G_{1} / U_{1} \times G_{2} / U_{2},
$$

and $L\left(E_{1}\right)=L_{\Lambda_{0}(1)}$ and $L\left(E_{2}\right)=L_{\Lambda_{0}(2)}$ by Lemma 3. Put $L_{1}=L_{\Lambda_{0}(1)}$ and $L_{2}=L_{\Lambda_{0}(2)}$. Let $\varphi_{1}: M \rightarrow N_{1}$ and $\varphi_{2}: M \rightarrow N_{2}$ be the natural projections. Note that there are holomorphic line bundles $L_{1}^{\prime}$ over $N_{1}$ and $L_{2}^{\prime}$ over $N_{2}$ such that $\varphi_{1}{ }^{*} L_{1}^{\prime}=L_{2}$ and $\varphi_{2}{ }^{*} L_{2}^{\prime}=L_{1}$ respectively. We thus have

$$
L\left(E_{1} \otimes L_{1}^{\prime-1}\right)=L_{1} \otimes L_{2}^{-1}=L\left(E_{2} \otimes L_{2}^{\prime-1}\right)^{-1} .
$$

Note also that the $\mathbb{P}^{1}$-bundle $X$ is given by $\mathbb{P}\left(1 \oplus L_{1} \otimes L_{2}{ }^{-1}\right)$. Put $G=G_{1} \times G_{2}$ and $U=U_{1} \times U_{1}$.

In cases (a) and (b), we call $X$ the $\mathrm{P}^{1}$-bundle associated to the triples $\left(\Pi, \Pi_{1}^{1}, \Pi_{0}\right)$, ( $\left.\Pi, \Pi_{1}^{2}, \Pi_{0}\right)$ of Dynkin diagrams. We also call $Y$ obtained as in Lemma 6.16 the compact complex manifold obtained from $X$ by blowing down associated to the triples $\left(\Pi, \Pi_{1}^{1}, \Pi_{0}\right),\left(\Pi, \Pi_{1}^{2}, \Pi_{0}\right)$ of Dynkin diagrams. Note that in this case $Y$ is almost homogeneous with respect to the complex Lie group $G$, since $E_{1} \otimes L_{1}^{\prime-1}-(0$-section $)=L_{1} \otimes L_{2}{ }^{-1}-$ (0-section) is an open $G$-orbit in $Y$, and $Y$ has a disconnected exceptional set which consists of two $G$-orbits $N_{1}, N_{2}$. Note also that $N_{1}, N_{2}$ are Kähler C-spaces associated to the pairs $\left(\Pi, \Pi_{1}^{1}\right),\left(\Pi, \Pi_{1}^{2}\right)$ respectively.

Theorem 6. Let $\left(\Pi, \Pi_{1}^{1}, \Pi_{0}\right)$ and $\left(\Pi, \Pi_{1}^{2}, \Pi_{0}\right)$ be triples of Dynkin diagrams, $X$ the $\mathbb{P}^{1}$-bundle associated to these triples of Dynkin diagrams and $Y$ the compact complex manifold obtained from $X$ by blowing down associated these triples of Dynkin diagrams. Then $Y$ is a Kähler manifold with positive first Chern class.

Proof. Let $g_{0}$ be the $G_{u}$-invariant Kähler metric on

$$
M=G / U=\mathbb{P}\left(E_{1} \otimes L_{1}^{\prime-1}\right)=\mathbb{P}\left(E_{2} \otimes L_{2}^{\prime-1}\right)
$$

corresponding to $8 \pi \delta_{\mathrm{m}}$ as in Fact 1 . Since we are in $G_{u}$-invariant situation, we can take $G_{u}$-invariant hermitian metrics $h_{1}$ on $E_{1} \otimes L_{1}^{\prime-1}$ and $h_{2}$ on $E_{2} \otimes L_{2}^{\prime-1}$. so that the $G_{u}$-invariant hermitian metric $h$ on the 
homogeneous line bundle

$$
L=L\left(E_{1} \otimes L_{1}^{\prime-1}\right)=L\left(E_{2} \otimes L_{2}^{\prime-1}\right)
$$

satisfies the first assumption of Lemma 6.16. Note also that the hermitian 2 -form $B$ on $M$ is $G_{u}$-invariant and corresponds to $4 \pi\left(-\Lambda_{0}(1)+\right.$ $\left.\Lambda_{0}(2)\right) \in \mathfrak{c}$ by Fact 2 . Thus $g_{t}=g_{0}-U(t) B$ is $G_{u}$-invariant and corresponds to

$$
4 \pi\left\{2 \delta_{\mathrm{m}}+U(t)\left(\Lambda_{0}(1)-\Lambda_{0}(2)\right\}\right.
$$

which is contained in $\mathfrak{c}_{+}$by Lemma 4 , and hence the second assumption of Lemma 6.16 is satisfied. By Lemma 4 , we also see that $g_{0}+(\ell+1) B=$ $\varphi_{1}{ }^{*} \underline{g}_{1}$ where $\underline{g}_{1}$ is a $G_{u}$-invariant Kähler metric on the Kähler C-space $N_{1}$ associated to the pair $\left(\Pi, \Pi_{1}^{1}\right)$ and $g_{0}-(k+1) B=\varphi_{2}{ }^{*} \underline{g}_{2}$ where $\underline{g}_{2}$ is a $G_{u}$-invariant Kähler metric on the Kähler C-space $N_{2}$ associated to the pair $\left(\Pi, \Pi_{1}^{2}\right)$, and hence the assumption of Lemma 6.17 is satisfied. Moreover the eigenvalues of $B$ with respect to $g_{0}$ are constant on $M$. Now, in view of Theorem 6.19, this finishes the proof.

Q.E.D.

Now let $\left(\Pi, \Pi_{0}\right)$ be an effective pair of Dynkin diagrams and $M=$ $G / U$ the Kähler C-space associated to $\left(\Pi, \Pi_{0}\right)$. Consider the EinsteinKähler metric $g_{0}$ on $G / U$ corresponding to $8 \pi \delta_{\mathrm{m}} \in \mathfrak{c}^{+}$with $\operatorname{Ric}\left(g_{0}\right)=g_{0}$ and a holomorphic line bundle $L_{\Lambda}$ on $G / U$ for $\Lambda \in Z_{\text {c }}$ with a $G_{u^{-}}$ invariant hermitian metric. Note that a unique $G_{u}$-invariant form in the first Chern class $c_{1}\left(L_{\Lambda}\right)$ is given by $(a .5)$. Let $B$ be the Ricci tensor of $L_{\Lambda}$ which is the $G_{u}$-invariant hermitian form on $M$ corresponding to $-4 \pi \Lambda \in \mathcal{c}$.

Lemma 7. Under the notations above, we have

$$
Q(x)=\operatorname{det}\left(1-x g_{0}^{-1} B\right)=\prod_{\alpha \in \Sigma_{\mathfrak{m}}^{+}}\left(1+\frac{(\Lambda, \alpha)}{\left(2 \delta_{\mathfrak{m}}, \alpha\right)} x\right) .
$$

Let $\rho$ be an automorphism of Dynkin diagram $\Pi$ such that $\rho^{2}=$ id and $\rho \neq$ id. It is known that if $\Pi$ is irreducible and it admits such an automorphism $\rho$, then $\Pi$ is of type $A_{n}(n \geqq 2), D_{n}(n \geqq 4)$ or $E_{6}$ (cf.[15]). Note also that if $\Pi$ has two connected components $\Pi(1)=$ $\left\{\alpha_{1}, \cdots, \alpha_{n}\right\}, \Pi(2)=\left\{\beta_{1}, \cdots, \beta_{n}\right\}$ and $\Pi(1), \Pi(2)$ are isomorphic by the map $\alpha_{i} \rightarrow \beta_{i}$, then the map $\rho: \Pi \rightarrow \Pi$ defined by $\rho\left(\alpha_{i}\right)=\beta_{i}, \rho\left(\beta_{i}\right)=$ $\alpha_{i}$ (for each $i$ ) is such an automorphism of $\Pi$, and from now on we consider this automorphism $\rho$ exclusively in the case when a Dynkin diagram $\Pi$ is reducible. A pair $\left(\Pi, \Pi_{0}\right)$ of Dynkin diagrams is said to be admissible for $\rho$ if $\rho\left(\Pi_{0}\right)=\Pi_{0}$. 
Lemma 8. Let $\left(\Pi, \Pi_{0}\right)$ be an admissible pair of Dynkin diagrams for an automorphism $\rho$ and assume that $\Lambda \in Z_{\mathrm{c}}$ satisfies $\rho(\Lambda)=-\Lambda$. Then

$$
Q(x)=\prod_{\alpha \in \Sigma_{\mathrm{m}}^{+}}\left(1+\frac{(\Lambda, \alpha)}{\left(2 \delta_{\mathrm{m}}, \alpha\right)} x\right)
$$

is an even function in $x$.

Corollary 9. Let $G / U$ be a Kähler $C$-space associated to an admissible pair $\left(\Pi, \Pi_{0}\right)$ for $\rho$. Put $2 \delta_{\mathrm{m}}=\sum_{\alpha \in \Pi-\Pi_{0}} a_{\alpha} \Lambda_{\alpha}$. Let $L_{\Lambda}$ be a holomorphic line bundle over $G / U$ such that $\rho(\Lambda)=-\Lambda$ and $\Lambda=$ $\sum_{\alpha \in \Pi-\Pi_{0}} b_{\alpha} \Lambda_{\alpha}$ with $\left|b_{\alpha}\right|<a_{\alpha}$ for each $\alpha \in \Pi-\Pi_{0}$. Then the $\mathbb{P}^{1}$. bundle $\mathbb{P}\left(1 \oplus L_{\Lambda}\right)$ over $G / U$ admits an Einstein-Kähler metric.

Example 10. In the following cases the $\mathbb{P}^{1}$-bundle $\mathbb{P}\left(1 \oplus L_{\Lambda}\right)$ over a Kähler C-space $M=G / U$ admits an Einstein-Kähler metric. The vertices contained in $\Pi_{0}, \Pi-\Pi_{0}$ of a Dynkin diagram $\Pi$ are denoted by $\circ, \times$ respectively.

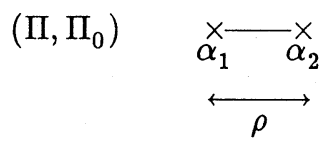

$2 \delta_{\mathrm{m}}=2\left(\Lambda_{\alpha_{1}}+\Lambda_{\alpha_{2}}\right)$. Put $\Lambda=\Lambda_{\alpha_{1}}-\Lambda_{\alpha_{2}}$. Then $\rho(\Lambda)=-\Lambda$. In this case $M=S L(3, \mathbb{C}) / B$, where $B$ is a Borel subgroup of $S L(3, \mathbb{C})$.

$$
\left(\Pi, \Pi_{0}\right)
$$

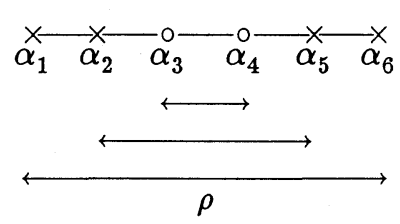

$2 \delta_{\mathrm{m}}=2 \Lambda_{\alpha_{1}}+4 \Lambda_{\alpha_{2}}+4 \Lambda_{\alpha_{5}}+2 \Lambda_{\alpha_{6}}$. Put $\Lambda=\Lambda_{\alpha_{1}}+\Lambda_{\alpha_{2}}-\Lambda_{\alpha_{5}}-\Lambda_{\alpha_{6}}$. Then $\rho(\Lambda)=-\Lambda$.

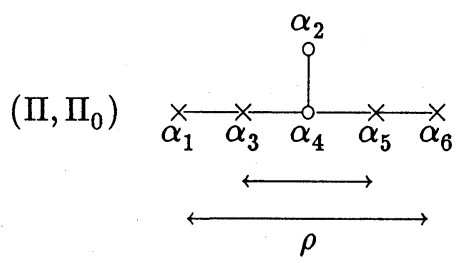

$$
2 \delta_{\mathrm{m}}=2 \Lambda_{\alpha_{1}}+4 \Lambda_{\alpha_{3}}+4 \Lambda_{\alpha_{5}}+2 \Lambda_{\alpha_{6}} . \text { Put } \Lambda=\Lambda_{\alpha_{3}}-\Lambda_{\alpha_{5}} . \text { Then } \rho(\Lambda)=-\Lambda \text {. }
$$




$$
\left(\Pi, \Pi_{0}\right)
$$

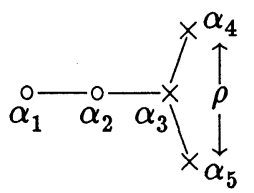

$2 \delta_{\mathrm{m}}=4 \Lambda_{\alpha_{3}}+2 \Lambda_{\alpha_{4}}+2 \Lambda_{\alpha_{5}}$. Put $\Lambda=\Lambda_{\alpha_{4}}-\Lambda_{\alpha_{5}}$. Then $\rho(\Lambda)=-\Lambda$.

$$
\left(\Pi, \Pi_{0}\right)
$$

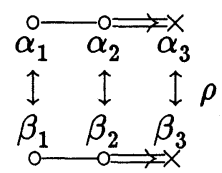

$2 \delta_{\mathrm{m}}=6 \Lambda_{\alpha_{3}}+6 \Lambda_{\beta_{3}}$. Put $\Lambda=\Lambda_{\alpha_{3}}-\Lambda_{\beta_{3}}$. Then $\rho(\Lambda)=-\Lambda$.

Now we consider triples $\left(\Pi, \Pi_{1}^{1}, \Pi_{0}\right),\left(\Pi, \Pi_{1}^{2}, \Pi_{0}\right)$ of Dynkin diagrams. These triples are said to be admissible for an automorphism $\rho$ of Dynkin diagram $\Pi$ if $\rho\left(\Pi_{0}\right)=\Pi_{0}$, and $\rho\left(\alpha_{0}(1)\right)=\alpha_{0}(2)$. Note that the holomorphic line bundle $L_{1} \otimes L_{2}{ }^{-1}=L\left(E_{1} \otimes L_{1}^{\prime}{ }^{-1}\right)=L\left(E_{2} \otimes L_{2}^{\prime-1}\right)^{-1}$ over $M=G / U$ is given by $L_{\Lambda}$, where $\Lambda=\Lambda_{0}(1)-\Lambda_{0}(2)$ and thus $\rho(\Lambda)=-\Lambda$.

Corollary 11. Let $\left(\Pi, \Pi_{1}^{1}, \Pi_{0}\right),\left(\Pi, \Pi_{1}^{2}, \Pi_{0}\right)$ be admissible triples of Dynkin diagrams for $\rho$. Then both the $\mathbb{P}^{1}$-bundle $X$ associated to these triples of Dynkin diagrams and the compact complex manifold $Y$ obtained from $X$ by blowing down associated to these triples admit Einstein-Kähler metrics.

Our compact Einstein-Kähler manifold $Y$ may be homogeneous. We give a necessary condition for an Einstein-Kähler metric on $\widehat{L}$ of the form (6.5.4) to be homogeneous.

Theorem 12 ([49]). Under the assumption of Theorem 6.7, assume further that $\operatorname{Ric}(\tilde{g})=\tilde{g}$. If $\tilde{g}$ is Riemannian homogeneous, the following holds.

(1) If the codimentions $D_{\min }=D_{\max }=1$, then $B=0$.

(2) If one of the codimensions $D_{\max }, D_{\min }$ is equal to 1 and the other $>1$, then the non-zero eigenvalues of $g_{0}^{-1} B$ are all equal.

(3) If both codimensions $D_{\min }, D_{\max }>1$, then the number of distinct non-zero eigenvalues of $g_{0}^{-1} B$ is 2 .

Corollary $13([49])$. Under the same assumption as in Corollary 9 , if the number of elements in $\Pi-\Pi_{0} \geqslant 3$, then the Einstein-Kähler metric on $Y$ is non-homogeneous. 
(1)

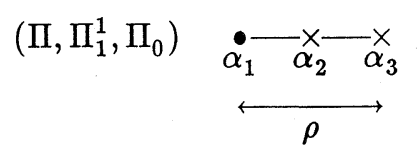

$\left(\Pi, \Pi_{1}^{2}, \Pi_{0}\right)$

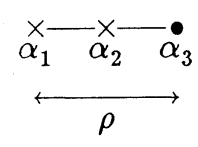

Note that $\Lambda=\Lambda_{\alpha_{1}}-\Lambda_{\alpha_{3}}$.

(2) $\left(\Pi, \Pi_{1}^{1}, \Pi_{0}\right)$

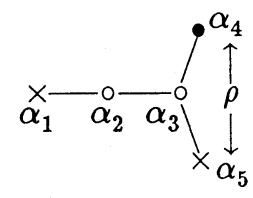

$\left(\Pi, \Pi_{1}^{2}, \Pi_{0}\right)$

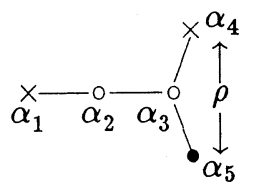

Note that $\Lambda=\Lambda_{\alpha_{4}}-\Lambda_{\alpha_{5}}$.

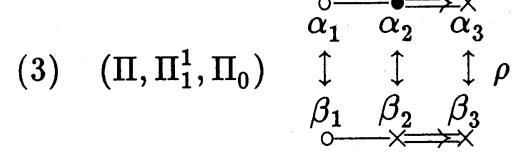

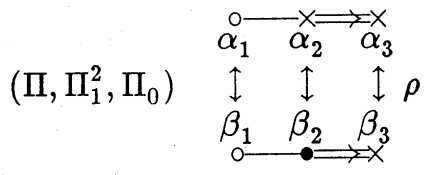

Note that $\Lambda=\Lambda_{\alpha_{2}}-\Lambda_{\beta_{2}}$.

(4)
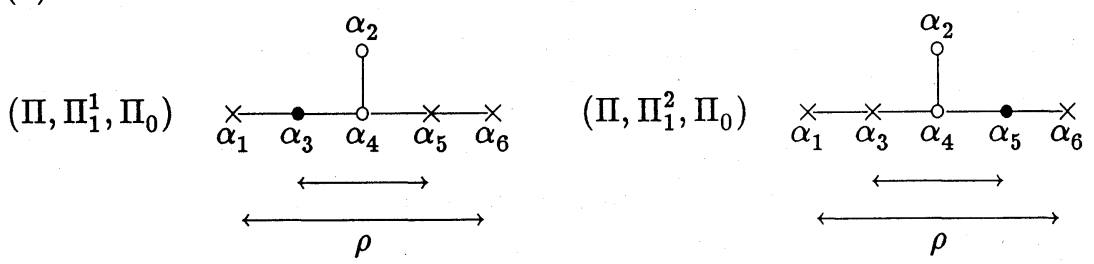

Note that $\Lambda=\Lambda_{\alpha_{3}}-\Lambda_{\alpha_{5}}$.

Example 14. In the following cases the compact Kähler manifold $Y$ admits a non-homogeneous Einstein-Kähler metric. The vertices contained in $\Pi_{0}, \Pi_{1}^{i}-\Pi_{0}, \Pi-\Pi_{1}^{i}$ of a Dynkin diagram $\Pi$ are denoted by $\circ, \bullet, \times$ for $i=1,2$ respectively as before. 


\section{References}

[1] D.N. Ahiezer, Dense orbits with two ends, Izv. Akad. Nauk SSSR, 41 (1977), 308-324 = Math. USSR Izvestija (Engl. transl.), 11 (1977), 293-307.

[2] M.F. Atiyah and R. Bott, The moment map and equivariant cohomology, Topology, 23 (1984), 1-28.

[3] T. Aubin, "Nonlinear analysis on manifolds", Springer, Berlin, New York, 1982.

[4] Réduction du cas positif de l'équation de Monge-Ampère sur les variété Kählériennes compactes à la démonstration d'une inégualité, J. Funct. Anal., 57 (1984), 143-153.

[5] S. Bando, An obstruction for Chern class forms to be harmonic, unpublished.

[6] —, The K-energy map, almost Einstein-Kähler metrics and an inequality of the Miyaoka-Yau type, Tohoku Math. J., 39 (1987), 231235.

[ 7 ] S. Bando and T. Mabuchi, Uniqueness of Einstein Kähler metrics modulo connected group actions, in "Algebraic Geometry, Sendai, 1985", Adv. Stud. in Pure Math. 10, Kinokuniya, Tokyo and North-Holland, Amsterdam, 1987, pp. 11-40.

[8] On some integral invariants on complex manifolds,I, Proc. Japan Acad., 62 (1986), 197-200.

[9] M. Berger, Sur les variétés d'Einstein compactes, C.R. IIIème Réunion Math. Expression Latine, Namur (1965), 35-55.

[10] N. Berline and M. Vergne, Zeros d'un champ de vecteurs et classes caractéristiques equivariantes, Duke Math. J., 50 (1983), 539-549.

[11] R.L. Bishop, A relation between volume, mean curvature and diameter, Amer. Math. Soc. Not., 10 (1963), p. 364.

[12] R. Bott, A residue formula for holomorphic vector fields, J. Differential Geometry, 1 (1967), 311-330.

[13] On the Lefschetz formula and the exotic characteristic classes, Symp. Math., 10 (1972), 95-105.

[14] _ On the characteristic classes of groups of diffeomorphisms, L'Enseignement Math., 23 (1977), 209-220.

[15] N. Bourbaki, "Groupes et algèbres de Lie, Chap. 4, 5 et 6", Hermann, Paris, 1968.

[16] J.P. Bourguignon, Invariants intégraux fonctionnels pour des équations aux dérivées partielles d'origine géométrique, in "Differerential Geometry, Peñiscola 1985", Lecture Notes in Math. 1209, Springer, Berlin, Heidelberg, New York, 1987, pp. 100-108.

[17] J.P. Bourguignon et al., "Preuve de la conjecture de Calabi", Astérisque 58, Soc. Math. France, 1978.

[18] E. Calabi, Extremal Kähler metrics II, in "Differential geometry and complex analysis", (ed. I. Chavel, H. M. Farkas) Springer, Berlin, 1985, pp. 95-114. 
[19] J. Cheeger and J. Simons, Differential characters and geometric invariants, in "Geometry and Topology", Lecture Notes in Math., 1167, Springer, Berlin, Heidelberg, New York, Tokyo, 1987.

[20] S.S. Chern and J. Simons, Characteristic forms and geometric invariants, Ann. Math., 99 (1974), 48-69.

[21] M. Demazure, Surfaces de Del Pezzo, in "Séminaire sur les singularités des surfaces, Palaiseu, France 1976-77", Lecture Notes in Math. 777, Springer, Heidelberg, New York, pp. 23-69.

[22] S.K. Donaldson, Anti-self-dual Yang-Mills connections over complex algebraic surfaces and stable vector bundles, Proc. London Math. Soc., 50 (1985), 1-26.

[23] J.J. Duistermaat and G.J. Heckman, On the variation in the cohomology of the symplectic form of the reduced phase space, Invent. Math., 69 (1982), 259-268.

[24] A. Fujiki and S. Nakano, Supplement to "On the inverse of monoidal transformation", Publ. R.I.M.S. Kyoto Univ., 7 (1971/72), 637-644.

[25] A. Futaki, An obstruction to the existence of Einstein Kähler metrics, Invent. Math., 73 (1983), 437-443.

[26] - On compact Kähler manifolds of constant scalar curvature, Proc. Japan Acad., 59 (1983), 401-402.

[27] - On a character of the automorphism group of a compact complex manifold, Invent. Math., 87 (1987), 655-660.

[28] The Ricci curvature of symplectic quotients of Fano manifolds, Tohoku Math. J., 39 (1987), 329-339.

[29] — "Kähler-Einstein metrics and integral invariants", Lecture Notes in Math., 1314, Springer, Berlin, Heidelberg, New York, Tokyo, 1988.

[30] A. Futaki and T. Mabuchi, An obstruction class and a representation of holomorphic automorphisms, in "Geometry and analysis on manifolds", Lectures Note in Math., 1339, Springer-Verlag, Berlin, Heidelberg, New York, Tokyo, 1988, pp. 127-141.

[31] A. Futaki and S. Morita, Invariant polynomials on compact complex manifolds, Proc. Japan Acad., 60 (1984), 135-142.

[32] - Invariant polynomials of the automorphism group of a compact complex manifold, J. Differential Geometry, 21 (1985), 135-142.

[33] A. Futaki and K. Tsuboi, On some integral invariants, Lefschetz numbers and induction maps, to appear in Tokyo J. Math..

[34] - Eta invariants and automorphisms of compact complex manifolds, in this volume.

[35] P. Griffiths and J. Harris, "Principles of Algebraic Geometry", WileyInterscience, New York, 1978.

[36] V. Guillemin and S. Sternberg, Geometric quantization and multiplicities of group representation, Invent. Math., 67 (1982), 515-538.

[37] J. Hano, Examples of projective manifolds not admitting Kähler metric with constant scalar curvature, Osaka J. Math., 20 (1983), 787-791. 
[38] K. Ishikawa and Y. Sakane, On complex projective bundles over a Kähler C-space, Osaka J. Math., 16 (1979), 121-132.

[39] V.A. Iskovskih, Fano 3-folds I, Izv. Akad. Nauk SSSR, Ser. Mat., 41 (1977), 516-562 = Math. USSR Izvestija (Engl. transl.), 11 (1977), 485-527.

[40] — Fano 3-folds II, Izv. Akad. Nauk SSSR, Ser. Mat., 42 (1978), 504-549 = Math. USSR Izvestija (Engl. transl.), 12 (1978), 469-506.

[41] J.L. Kazdan, Gaussian and scalar curvature, an update, in "Seminar on differential geometry (ed. S.T.Yau)", Princeton Univ. Press, New Jersey, 1982, pp. 185-191.

[42] J.L. Kazdan and F.W. Warner, Curvature functions for compact 2-manifolds, Ann. Math., 99 (1974), 14-47.

[43] J.L. Kazdan and F.W. Warner, Scalar curvature and conformal deformations of Riemannian strucure, J. Differential Geometry, 10 (1975), $113-134$.

[44] S. Kobayashi, On compact Kähler manifolds with positive definite Ricci tensor, Ann. Math., 74 (1961), 570-574.

[45] S. Kobayashi, Negative vector bundles and complex Finsler structures, Nagoya Math. J., 57 (1975), 153-166.

[46] - "Differential geometry of complex vector bundles", Publ. Math. Soc. Japan 14, Iwanami, Tokyo and Princeton Univ. Press, Princeton, 1987.

[47] S. Kobayashi and K. Nomizu, "Foundations of differential geometry", Vol. II, Interscience, Wiley, New York, London, Sydney, 1969.

[48] N. Koiso and Y. Sakane, Non-homogeneous Kähler-Einstein metrics on compact complex manifolds, in "Curvature and topology of Riemannian manifolds,", Lecture Notes in Math., 1201, Springer, Berlin, Heidelberg, New York, Tokyo, 1986.

[49] N. Koiso and Y. Sakane, Non-homogeneous Kähler-Einstein metrics on compact complex manifolds II, Osaka J. Math., 25 (1988), 933-959.

[50] M. Koitabashi, Automorphism groups of rational surfaces, M.A. thesis (Japanese), Osaka Univ. (1986).

[51] Y. Kollár and T. Matsusaka, Riemann-Roch type inequalities, Amer. J. Math., 105 (1983), 229-252.

[52]. A. Lichnerowicz, "Géométrie des groupes de transformations", Dunod, 1958.

[53] - Sur les transformations analytiques des variétés kählériennes, C. R. Acad. Sci. Paris, 244 (1957), 3011-3014.

[54] - Isométrie et transformations analytique d'une variété kählérienne compacte, Bull. Soc. Math. France, 87 (1959), 427-437.

[55] — Variétés kählériennes et première classe de Chern, J. Differential Geometry, 1 (1967), 195-224.

[56] M. Lübke, Stability of Einstein-Hermitian vector bundles, Manuscripta Math., 42 (1983), 245-257. 
[57] T. Mabuchi, K-energy maps integrating Futaki invariants, Tohoku Math. J., 38 (1986), 575-593.

[58] — Einstein-Kähler forms, Futaki invariants and covex geometry on toric Fano varieties, Osaka J. Math., 24 (1987), 705-737.

[59] An algebraic character associated with the Poisson brackets, in this volume.

[60] _ Some symplectic geometry on compact Kähler manifolds (I), Osaka J. Math., 24 (1987), 227-252.

[61] J. Marsden and A. Weinstein, Reduction of symplectic manifolds with symmetry, Reports on Math. Physics, 5 (1974), 121-130.

[62] T. Matsusaka, Polarized varieties with a given Hilbert polynomial, Amer. J. Math., 94 (1972), 1027-1077.

[63] Y. Matsushima, Sur la structure du groupe d'homéomorphismes analytiques d'une certaine variété kählérienne, Nagoya Math. J., 11 (1957), 145-150.

[64] — Remarks on Kähler-Einstein manifolds, Nagoya Math. J., 46 (1972), 161-173.

[65] B.G. Moisezon, On $n$-dimensional compact varieties with $n$ algebraically independent meromorphic functions I, II, III, Izv. Akad. Nauk. SSSR, 30 (1966), 133-174; 345-386; 621-656.

[66] S. Mori and S. Mukai, Classification of Fano 3 -folds with $B_{2} \geq 2$, Manuscripta Math., 36 (1981), 147-162.

[67] S.B. Myers, Riemannian manifolds with positive mean curvature, Duke Math. J., 8 (1941), 401-404.

[68] S. Nakano, On the inverse of monoidal transformation, Publ. R.I.M.S. Kyoto Univ., 6 (1970/71), 483-502.

[69] T. Nitta and M. Takeuchi, Contact structures on twister spaces, J. Math. Soc. Japan, 39 (1987), 139-162.

[70] Y. Sakane, On non-singular hyperplane sections of some hermitian symmetric spaces, Osaka J. Math., 22 (1985), 107-121.

[71] — Examples of compact Kähler-Einstein manifolds with positive Ricci curvature, Osaka J. Math., 31 (1986), 585-617.

[72] S.M. Salamon, Quaternionic Kähler manifolds, Invent. Math., 67 (1982), 143-171.

[73] Y.-T. Siu, "Lectures on Hermitian-Einstein metrics for stable bundles and Kähler-Einstein metrics", DMV seminar '86, Band 8, Birkhäuser, Basel, Boston, Stuttgart, 1987.

[74] G. Tian, On Kähler-Einstein metrics on certain Kähler manifolds with $C_{1}(M)>0$, Invent. Math., 89 (1987), 225-246.

[75] G. Tian and S.-T. Yau, Kähler-Einstein metrics on complex surfaces with $C_{1}>0$, Commun. Math. Phys., 112 (1987), 175-203.

[76] S.-T. Yau, On the curvature of compact Hermitian manifolds, Invent. Math., 25 (1974), 213-239.

[77] S.-T. Yau, On the Ricci curvature of a compact Kähler manifold and the complex Monge-Ampère equation I, Comm. Pure and Appl. Math., 
31 (1978), 339-411.

\author{
A. Futaki \\ Department of Mathematics \\ Tokyo Institute of Technology \\ O-okayama, Meguro, Tokyo 152 \\ Japan \\ T. Mabuchi \\ College of General Education \\ Osaka University \\ Machikaneyama, Toyonaka 560 \\ Japan \\ Y. Sakane \\ Department of Mathematics \\ Osaka University \\ Machikaneyama, Toyonaka 560 \\ Japan
}

